\title{
Promoting awareness of fat, fruit and vegetable intake
}

Citation for published version (APA):

Oenema, A. (2004). Promoting awareness of fat, fruit and vegetable intake. [Doctoral Thesis, Maastricht University]. Maastricht University. https://doi.org/10.26481/dis.20040513ao

Document status and date:

Published: 01/01/2004

DOI:

10.26481/dis.20040513ao

Document Version:

Publisher's PDF, also known as Version of record

\section{Please check the document version of this publication:}

- A submitted manuscript is the version of the article upon submission and before peer-review. There can be important differences between the submitted version and the official published version of record.

People interested in the research are advised to contact the author for the final version of the publication, or visit the DOI to the publisher's website.

- The final author version and the galley proof are versions of the publication after peer review.

- The final published version features the final layout of the paper including the volume, issue and page numbers.

Link to publication

\footnotetext{
General rights rights.

- You may freely distribute the URL identifying the publication in the public portal. please follow below link for the End User Agreement:

www.umlib.nl/taverne-license

Take down policy

If you believe that this document breaches copyright please contact us at:

repository@maastrichtuniversity.nl

providing details and we will investigate your claim.
}

Copyright and moral rights for the publications made accessible in the public portal are retained by the authors and/or other copyright owners and it is a condition of accessing publications that users recognise and abide by the legal requirements associated with these

- Users may download and print one copy of any publication from the public portal for the purpose of private study or research.

- You may not further distribute the material or use it for any profit-making activity or commercial gain

If the publication is distributed under the terms of Article $25 \mathrm{fa}$ of the Dutch Copyright Act, indicated by the "Taverne" license above, 


\section{Promoting awareness of fat, fruit and vegetable intake}





\section{Promoting awareness of fat, fruit and vegetable intake}

\section{Proefschrift}

ter verkrijging van de graad van doctor aan de Universiteit Maastricht,

op gezag van Rector Magnificus, Prof.mr. G.P.M.F. Mols volgens het besluit van het College van Decanen, in het openbaar te verdedigen op donderdag 13 mei 2004 om 14.00 uur

door

Anke Oenema 


\section{Promotor:}

Prof.dr.ir. J. Brug

\section{Beoordelingscommissie:}

Prof.dr. N.K. de Vries (voorzitter)

Dr. M.K. Campbell (University of North Carolina, Verenigde Staten)

Prof.dr. I, De Bourdeaudhuif (Universiteit Gent, Belgie)

Prof.dr. A.T.M. Jansen

Prof.dr. H. de Vries

ISBN 90-77595-16-3

Interior and cover design by Anna Bosselaar.

Printed by Optima Grafische Communicatie, Rotterdam.

The study presented in this thesis was performed within the Nutrition and Toxicology Research Institute Maastricht (NUTRIM) which participates in the Graduate School VLAG (Food Technology. Agrobiotechnology. Nutrition and Health Sciences), accredited by the Royal Netherlands Academy of Arts and Sciences.

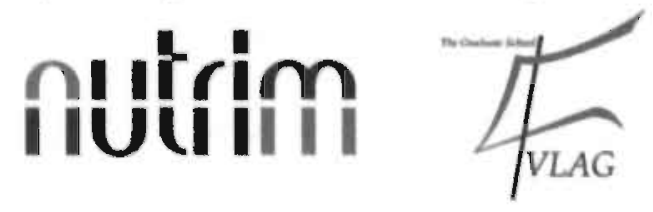

The studies described in this thesis were financially supported by the Netherlands Organisation for Health Research and Development (ZonMW, projectnumber 2100.0001).

A grant for the publication of this thesis by the Hendrick Nannes \& Catrijn Epes Leen in Bolsward is gratefully acknowledged. 


\section{Contents}

Chapter 1 Introduction

Chapter 2 The use and impact of computer-tallored nutrition education

Chapter 3 Web-based tailored nutrition education: Results of a randomised controlled trial

Chapter 4 Feedback strategies to raise awareness of personal dietary intake: Results of a randomised controlled trial

Chapter 5 Short-term efficacy of a web-based computer-tailored nutrition intervention: main effects and mediators

Chapter 6 Long-term efficacy of a web-based computer-tailored nutrition intervention

Chapter 7 Exploring the occurrence and nature of comparison of one's own perceived dietary fat intake to that of self-selected others

Chapter 8 An exploration of the effects of positive and negative role model stories on awareness, intention and fruit intake

Chapter 9 General discussion

References

Summary

Samenvatting

Dankwoord 


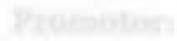

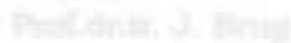

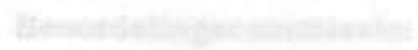

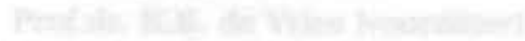

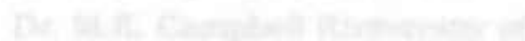

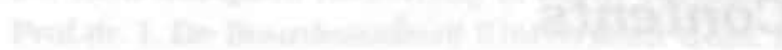

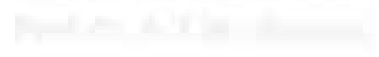

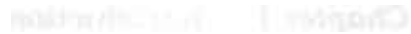




\section{Introduction}

Cardiovascular diseases and cancers are the most common chronic diseases and the major causes of death in the Netherlands and in most other Western countries. Behavioural factors associated with an increased risk of these diseases include high intakes of saturated fat and low intakes of fruit and vegetables. Diets high in saturated fat and low in fruit and vegetables are very common in the Netherlands. Since changes in dietary patterns could contribute to the prevention of these chronic diseases, there is a need for effective intervention strategies to motivate people to adopt diets low in saturated fat and high in fruit and vegetables.

In this thesis, various possible intervention strategies to motivate people to adopt more healthful diets are evaluated for their efficacy. The interventions focus specifically on increasing awareness of personal intake levels of fat, fruit and vegetables. since lack of awareness of personal dietary intake has been found to be an important barrier for the motivation to change behaviour (Brug, Van Assema, Kok. Lenderink, \& Glanz, 1994; Glanz, Brug, \& Van Assema, 1997: Lechner. Brug. \& De Vries, 1997; Weinstein, 1988). The interventions evaluated in this thesis were developed from a problem-driven approach and the studies fit in with the steps of planned health education (Kok, Schaalma, De Vries, Parcel, \& Paulussen, 1996; Kok, Schaalma, \& Brug, 2000). The studies in this thesis were also intended to contribute to the state of scientific knowledge about computer-tallored nutrition education and the application of social comparison and modelling processes in nutrition education. This introductory chapter provides an overview of the steps in the process of planned health education that guided the choice of the behavioural goals, and the development and evaluation of the interventions. 


\section{Planned health education}

In the process of planned health education, five phases are distinguished (Figure 1.1). The process starts with a problem analysis, which is intended to identify a health problem that, from a public health perspective, is important enough to address. A health problem is important enough if it imposes a major burden on quality of life, disease prevalence and/or mortality, for the public in general or for specific risk groups. After important health problems and risk groups have been identified, behavioural and environmental risk factors for these diseases, as well as the differential prevalence rates of risk behaviours among specific population groups, have to be identified in the phase of risk behaviour analysis. Once behavioural risk factors have been identified, the causal factors or determinants of these risk behaviours have to be identified in the analysis of behavioural determinants. This step is important, since inducing behaviour change is only possible by influencing its determinants. It is therefore particularly important to identify modifiable determinants. Once important and modifiable determinants have been identified, the next step is intervention development. Intervention development should be informed by behaviour change theory and should aim at enabling changes in the behavioural determinants, and hence in actual behaviour. Identified methods for inducing changes in determinants have to be translated into deliverable intervention strategies and have to be implemented and evaluated for their efficacy. If interventions are found to be effective and cost-effective, they can be disseminated on a larger scale. The remainder of this chapter describes the steps leading to intervention development and evaluation in more detail.

Figure 1.1. Model of planned health education (From: Kok et al., 2000)

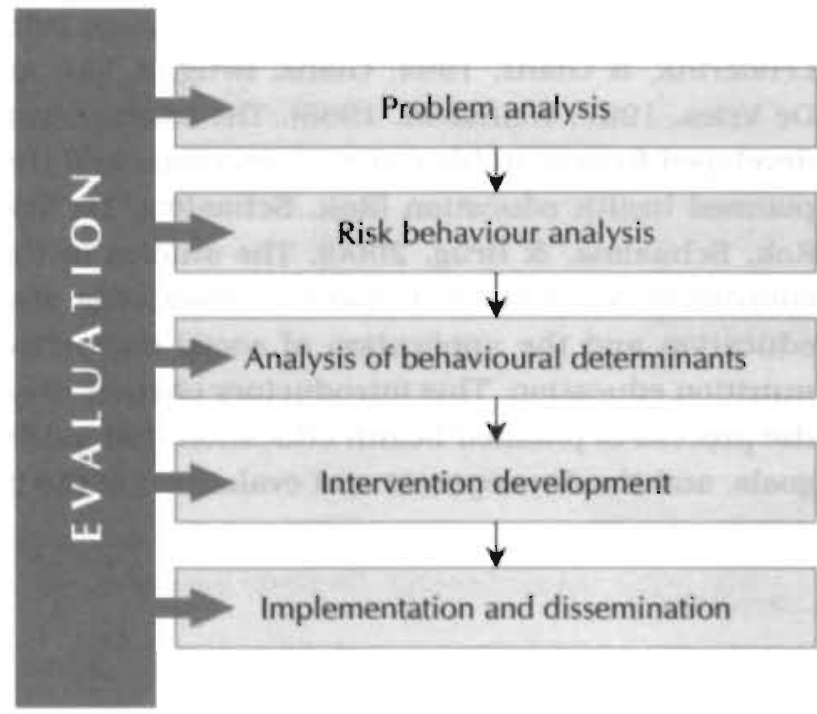




\section{Problem analysis}

Cardiovascular diseases (CVD) and cancer are the most common chronic diseases and the major causes of mortality in Western countries (Centraal Begeleidingsorgaan voor de Intercollegiale Toetsing [CBO]. 1998; Murray \& Lopez, 1997; Van Oers, 2002). In 2000 and 2001, cardiovascular diseases accounted for $35 \%$ of total mortality in the Netherlands, while cancers accounted for $27 \%$ (Koek, Van Leest. Verschuren. \& Bots, 2003; Van Oers, 2002). Over the past decade, there has been a decline in CVD mortality, which is most probably the result of improvements in treatment and life-style. The incidence of CVD, however, did not decline, resulting in an increased prevalence (Van Oers, 2002). There has been a decrease in total cancer mortality, even though mortality for some types of cancer increased (melanoma and cancer of the head and neck region). Cancer incidence has increased slightly over the past decade, which is due to population growth and screening and early detection programmes (Visser, Coebergh, Van Dijck, \& Siesling, 2002). In all, how. ever, the decrease in cancer mortality and the increase in cancer incidence has resulted in an increase in cancer prevalence (Van Dijck, Coebergh. Siesling. \& Visser, 2002; Vereniging van Integrale Kankercentra, 2003: Van Oers, 2002). It is estimated that, if nothing changes, future decades will see the prevalence of CVD and cancer increasing by 25 to $65 \%$ in the Netherlands, due to the ageing population (Van Oers, 2002). Cardiovascular diseases will remain the leading cause of mortality in Western countries (Murray \& Lopez, 1997). Not only do these diseases represent a major burden of disease and mortality, they are also associated with disability and loss of quality of life (Van Oers, 2002). Even though there have been. and most probably will continue to be, improvements in the medical treatment of CVD and cancer, the greatest gain in public health is to be expected from primary prevention of these diseases (Van Oers, 2002).

\section{Risk behaviour analysis}

High blood pressure, high cholesterol levels, diabetes and obesity have been identified as risk factors for CVD, and obesity has also been identified as a risk factor for some types of cancer. Diet has been identified as one of the behavioural risk factors for CVD and cancer, with high saturated fat intake and low fruit and vegetable intake being associated with an increased risk of CVD, cancer and obesity (CBO, 1998: Van Oers, 2002; World Cancer Research Fund, 1997: World Health Organization, 2003). It is estimated that 5\% of annual deaths in the Netherlands are associated with a high saturated fat intake and another $5 \%$ with low fruit and vegetable intake (Van Oers, 2002). Other important behavioural factors associated with the risk of CVD and cancer are smoking. physical inactivity and excessive alcohol use (CBO, 1998: Ezzati, Lopez, Rodgers, Vander Hoorn, \& Murray, 2002: Van Oers, 2002). 


\section{Saturated fat intake and cardiovascular disease risk}

Cardiovascular diseases are the result of a deteriorated condition of the arteries. called arteriosclerosis. One factor that plays a role in the process leading to arteriosclerosis and subsequent CVD is serum cholesterol. Two types of serum cholesterol are relevant in this respect: low-density lipoprotein (LDL-cholesterol), a high serum level of which is associated with a high risk of coronary heart disease (CHD), and high-density lipoprotein (HDL-cholesterol), a high serum level of which is associated with a low risk of $\mathrm{CHD}$. Both types of cholesterol contribute to the total serum cholesterol level. The ratio between total serum cholesterol and HDL-cholesterol is the best indicator of CHD risk. A high ratio, implying a high serum level of LDLcholesterol and a low serum level of HDL-cholesterol, indicates a high risk of CHD (CBO, 1998).

High serum cholesterol levels can be genetically determined (primary hyperlipidemia) or acquired (secondary hyperlipidemia). Secondary hyperlipidemia is much more common than primary hyperlipidemia and life-style factors like consumption of a high saturated fat and high cholesterol diet. excessive alcohol use, smoking and physical inactivity are amongst the causes (CBO, 1998).

Serum cholesterol levels increase with the intake of dietary fat. Saturated fats contribute to a higher LDL-cholesterol level, whereas unsaturated fats contribute to a higher HDL-cholesterol level. Fats of animal origin (meat, dairy products) and some vegetable fats (e.g.. coconut oil. palm oil. cocoa) are high in saturated fat and thus increase LDL-cholesterol level. Like saturated fat, trans fatty acids (naturally found in the meat of ruminants and artificially produced as a by-product in the process of solidifying liquid oils) increase LDL-cholesterol level, but also decrease HDL-cholesterol level. thus causing an unfavourable ratio between total and HDLcholesteroll. Vegetable oils, nuts, fatty fish and certain fat products enriched with linoleic acid are high in unsaturated fat and increase HDL-cholesterol levels, resulting in a favourable ratio between total and HDL cholesterol (CBO, 1998; Willet. 1994).

A high intake of (saturated) fat is also associated with an increased risk of overweight and obesity, and of type 2 diabetes (Rolls \& Shide, 1992), which are also risk factors for CVD. Thus, apart from an effect on serum cholesterol levels, there may also be an indirect effect of fat intake on CVD risk, through overweight and obesity.

\section{Fruit and vegetable intake and cardiovascular disease risk}

There is growing evidence for a protective effect of fruit and vegetable consumption against CVD risk (Joshipura, Hu, Manson, Stampfer, Rimm, et al., 2001: Ness \& Powles, 1997), although few prospective cohort studies examining the associations between fruit and vegetables and CVD risk are available. The mechanisms by which fruit and vegetables exert their protective effect against CVD are not yet fully understood. The antioxidants in fruit and vegetables may lead to a reduced oxida- 
tion of cholesterol in the arteries, thus preventing arteriosclerosis. Other nutrients in fruit and vegetables, like folate, fibre and minerals may also contribute to the risk decreasing effect (CBO, 1998; Joshipura et al., 2001). Furthermore, because of their low caloric density and high fibre content, fruit and vegetables may prevent overweight and obesity, thereby eliminating a risk factor for CVD (CBO, 1998; World Cancer Research Fund, 1997).

\section{Saturated fat intake and cancer risk}

The relationship between fat intake and cancer risk has been studied extensively. particularly in relation to the most common cancers for which this relationship was expected, that is, breast, colon and prostate cancer. Prospective cohort studies have not found straightforward associations between the intake of total fat, saturated fat, unsaturated fat or red meat and cancer risk, and no strong associations have been found (Key, Allen, Spencer, \& Travis, 2002; Van den Brandt, Dagnelie. \& Von Meyenfeldt, 1999; Zock, 2001). The current state of evidence suggests that there is no strong support for a direct relationship between high intakes of total and saturated fat and the risk of cancer.

\section{Fruit and vegetable intake and cancer risk}

Fruit and vegetable consumption have been studied as protective factors against various types of cancer. At present, the evidence from epidemiological studies indicates a probable protective effect of fruit and vegetable consumption against oral cavity. pharynx, oesophagus, stomach and colorectal cancer and a possible protective effect against lung cancer (Key et al., 2002; World Cancer Research Fund, 1997).

The influence of saturated fat. fruit and vegetable intake on cancer risk may also be more indirect, through their effects on obesity. Obesity has been identified as a risk factor for oral cavity, pharynx, oesophagus, colorectal, postmenopausal breast, endometrial and kidney cancer (Key et al., 2002; World Cancer Research Fund, 1997).

\section{Recommended intake levels for dietary fat, fruit and vegetables}

It is generally accepted that dietary recommendations regarding fat, fruit and veg. etable intake should be provided to the public as to reduce the risk of CVD and cancer: The World Cancer Research Fund (1997) has formulated universal dietary recommendations, which apply to the prevention of cancer as well as CVD, and which have to be translated into practical national guidelines. The recommendations are as follows: 'Eat 400-800 grams of a variety of fruit and vegetables a day. all year round': 'Limit the consumption of fatty foods, particularly those of animal origin": 'Choose modest amounts of appropriate vegetable oils'; 'Avoid being underweight or overweight' and 'Limit weight gain in adulthood to less than $5 \mathrm{~kg}$ '. 
The dietary guidelines issued by the Health Council of the Netherlands include recommendations for (1) a total fat intake of 20-40 percent of total energy intake (\%en) for non-overweight adults with a constant body weight and a total fat intake of 20 to $30-35 \%$ for overweight adults or those at risk of becoming overweight; (2) a maximum saturated fat intake of 10 \%en for all adults; (3) a maximum intake of trans fatty acids of 1 \%en for all adults. Percent energy (\%en) indicates the proportion of the total daily intake of energy that is contributed by each type of fat. These guidelines are the result of a trade-off between recommending the ideal healthy diet and the feasibility of the guidelines in the Dutch situation. The guidelines diverge from guidelines in other countries in that the recommended maximum intake of total fat is higher than that in many other countries and that the recommended total fat intake depends on body weight (Health Council of the Netherlands, 2001). It should, however, be kept in mind that at present, about $40 \%$ of the Dutch adult population are overweight and that a (large) majority of the population can be regarded as at risk of becoming overweight (Health Council of the Netherlands, 2003).

The guidelines for fruit and vegetable intake in the Netherlands are: 'Eat at least 200 grams of vegetables and two servings (250-300 grams) of fruit per day'. These recommendations for a daily intake of at least 450-500 grams of fruit and vegetables each day are comparable with the ' 5 a day' message that is more common in countries like the US and the UK. These recommended amounts of fruit and vegetables are a minimum, and higher intake levels may yield greater health benefits.

\section{Prevalence of risk behaviour: intake of fat. fruit and vegetahles in the} Netherlands

The most recent data about food consumption patterns in the Netherlands is available through the third National Food Consumption Survey (NFCS) held in 1997/ 1998 (Voedingscentrum, 1998). The average intake of total fat in the entire study population, aged 1 to 75 , was $35.9 \%$ en and that of saturated fat $14.2 \%$ en. The average total fat intake for women aged 22 to 65 was $37 \%$ en and that of saturated fat 14.7 \%en. For men in this age group, average total fat intake was $36.6 \%$ en and that of saturated fat $14.4 \%$ en. There was a decreasing trend in total fat intake between the first NFCS in 1987/1988 (which showed an average intake of $38.7 \%$ en in the entire study population) and the third one in 1998. Saturated fat intake declined between the first and the second NFCS (from 14.5\%en to $12.9 \%$ en), but increased between the second and the third one, to almost the same level as in the first NFCS in 1987/1988. These trends were found for all age and gender groups. Even though over the years there appears to have been some progress towards a lower total fat consumption, it is clear that the intake level of saturated fat far exceeds the recommended level of $10 \%$ en saturated fat. It is estimated that 75 to $90 \%$ of the Dutch population consumes larger than recommended amounts of saturated fat (Health Council of the Netherlands, 2001; Van Oers, 2002).

Average daily intake of vegetables in the total NFCS study population was 123 
grams, and $93 \%$ of the respondents consumed vegetables on at least one of the record days. The average datly vegetable intake in 1992 was 128 grams and that in 1987/1988 141 grams, while the proportions of vegetable consumers were $94 \%$ and $92 \%$ respectively. In the 22 to 65 age group, average vegetable intake ranged from 125 to 130 grams for women and men under the age of 50 and from 160 to 170 grams for women and men aged 50 years and older. These figures show that the average vegetable intake is far below the recommended intake level, that this is more obvious for those younger than 50 years, and that there is a downward trend in vegetable consumption.

The average intake of fruit was 105 grams (fruit juice not included) per day in the total studied population aged 1 to 75 , and declined from 125 grams in $1987 /$ 1988 to 114 grams in 1992 . Seventy-five percent of the respondents consumed fruit on at least one of the record days in the 1997/1998 NFCS. In the 22 to 65 age group, average intake of fruit and fruit juice was 163 grams per day for women and 133 grams for men under the age of 50 and 203 grams for women and 167 grams for men aged 50 years and older. There is a downward trend in fruit consumption, which may, however, be compensated by the use of fruit juice. However, even if the amounts of fruit and fruit juice are combined for the 22 to 65 age group, average intake is still below the recommended level. Moreover, $25 \%$ of respondents did not consume any fruit on the days of their dietary record (Voedingscentrum. 1998).

The results of the NFCS indicate that the average consumption patterns of saturated fat, fruit and vegetables are not in line with recommended intake levels. It is thus most likely that large parts of the Dutch adult population engage in behaviour that increases the risk of CVD and cancer. If the Dutch population complied with the recommendations for saturated fat, fruit and vegetable intake, 4,500 new cases of cancer and 5.000 deaths caused by CVD could be prevented (Schram, Maas, Poos, \& Jansen, 2001).

Summarised findings of the problem analysis and behavioural risk factor analysis

CVD and cancer are highly prevalent diseases and leading causes of mortality in the Netherlands. Due to the ageing population, their prevalence and mortality are even expected to increase in the future. Dietary intakes of saturated fat. fruit and vegetables have been found to be among the behavioural risk factors associated with these diseases, either directly or indirectly through overweight and obesity. Moderate intake of saturated fat and ample intake of fruit and vegetables can reduce the risk of these diseases considerably. indicating that an effective preventive action is available. A majority of the Dutch population across genders and most age groups engage in dietary risk behaviour.

Two important health problems have been identified, with a clear behavioural component associated with the risk. Risk behaviour appears to be prevalent, and reducing risk behaviour is likely to contribute to disease prevention. Therefore it seems important to invest in the development of interventions to improve dietary 
intake patterns. Even though the risk behaviour is prevalent in all age groups, the prevalence of the diseases increases with age. The focus of the present thesis is mainly on adults, although other age groups could have been targeted.

\section{Analysis of behavioural determinants}

The phase of analysis of behavioural determinants is an important one, since behaviour cannot generally be changed directly. Merely telling people to stop engaging in a particular risk behaviour or to adopt a more healthy behaviour is not likely to result in behaviour change. Behaviour can be more successfully changed by influencing its determinants. Determinants are causal factors that induce an individual to engage in a particular behaviour. An analysis of the behavioural determinants reveals the most important determinants for a particular behaviour and ideally also provides insight into what determinants could be changed by means of an intervention. This section describes the analysis of the behavioural determinants of dietary intake of fat. fruit and vegetables.

\section{Explaining dietary behaviour: searching for behavioural determinants}

Several types of determinants can be distinguished for dietary intake, depending on the perspective from which diet is studied. Biological determinants focus on the biological mechanisms behind food choice. An example is the innate preference for sweet tastes and aversion to bitter tastes, leading to the choice of sweet foods and avoidance of bitter foods. Determinants of learning to like these tastes are studied from a learning theory perspective. Environmental determinants relate to the physical environment. for example the availability of healthy or unhealthy foods, but may aiso include the social environment, the economic environment or the neighbourhood. Socio-cultural determinants focus on the norms and customs related to food in societies and cultures. Social psychological determinants focus on the more deliberate cognitions that determine food choice. These cognitions are supposed to be under conscious control and may therefore be most likely to be modifiable by means of health education interventions. The focus of this thesis is thus on interventions targeting these social psychological determinants.

\section{Social cognition models and determinants}

Social cognition models assume that (health) behaviour is the result of a rational decision making process (Conner \& Norman, 1995). Even though different models emphasise different aspects of the decision making process, there is an overlap in the underlying constructs that play a role in most of these models. Most models focus on the motivational processes leading to behaviour. Motivational models assume that the determinant that is most proximal and most important to the performance of a particular behaviour is the intention to engage in that behaviour 
(e.g., the intention to eat low-saturated fat foods precedes the actual intake of such foods). The Theory of Reasoned Action (TRA), later extended and changed into the Theory of Planned Behaviour (TPB) and the Attitude, Social influence and self-Eftcacy (ASE) model are examples of such motivational models (Ajzen. 1988; De Vries. Dijkstra, \& Kuhlman. 1988; De Vries \& Mudde, 1998; Fishbein \& Ajzen, 1975). which both have been used in explaining dietary intake.

Attitudes, social influences and self-efficacy expectations or perceived behavioural control are the determinants most proximal to intention. An attitude is the overall evaluation of a behaviour, determined by the balance between the most salient expected advantages and disadvantages of the behaviour. Social influence consists of subjective norms, that are. beliefs about what others want you to do. perceived social pressure or support and direct modelling of others' behaviour. Selfefficacy expectations or perceived behavioural control refer to the confidence one has in being able to perform a behaviour in situations with varying levels of diffculty. Apart from the influence on behavioural intention, self-efficacy expectations also relate directly to behaviour (Ajzen, 1988). A positive attitude, positive and supportive social influence and high self-efficacy expectations about performing a particular behaviour are expected to result in a positive intention to engage in that behaviour. Such a positive intention to engage in a particular behaviour is then likely to result in actual performance of the behaviour. Even though these models have been widely used to explain health behaviours, the models lack a special emphasis on perceptions of health risk and health risk behaviour. Perceived risk. perceived vulnerability and perceived severity of a negative health outcome are factors that are given a central role in the Health Belief Model and the Protection Motivation Theory (Boer \& Seydel, 1995: Sheeran \& Abraham, 1995). Adding these aspects as specific beliefs in the attitude concept to explain health risk behaviours may contribute to a more specific prediction of intention.

Several determinants have been suggested as important additions to increase the predictive validity of the above models. Suggested additional determinants include moral norm (for behaviours with a moral or ethical dimension, such as consuming organic or genetically modified foods), anticipated regret, self-predictions, self-identity, past behaviour, habit, exposure, environmental factors and emotional factors (Norman \& Conner, 1995; Conner \& Armitage. 1998).

Given that the determinants predict behaviour, the desired health behaviour can be induced or modified by influencing these determinants. Therefore, interventions should be designed to influence the important cognitive factors related to a particular behaviour (Conner \& Norman. 1995).

\section{Determinants of fat, fruit and vegetable consumption}

Analysing the determinants of fat, fruit and vegetable consumption can be done by searching the existing literature on determinant research, by performing additional determinant studies, or both. The present thesis builds upon the existing literature on determinant research. 
Attitude, taste, health beliefs, normative beliefs, modelling and self-efficacy expectations have been found to be the most important determinants for the intention to consume and the actual consumption of (saturated) fat, with an explained variance of 20-50\% (Baranowski, Cullen, \& Baranowski, 1999; Brug \& Van Assema, 2001: Glanz, Basil, Maibach, Goldberg, \& Snyder, 1998; Stafleu, De Graaf, Van Staveren, \& Schroots, 1991). Explained variance has proved to be greater for intention than for behaviour. Self-efficacy, attitude, knowledge about recommended intake levels and being in the habit of eating large amounts of fruit and vegetables from childhood onward have been found to be the most important determinants for the intention to consume and the actual intake of fruit and vegetables (Brug. Lechner, \& De Vries, 1995: Glanz et al., 1998: Krebs-Smith, Heimendinger, Patterson. Subar, Kessler, \& Pivonka, 1995: Lechner et al., 1997: Van Duyn, Kristal, Dodd, Campbell, Glanz, \& Subar, 2001).

Even though these main determinants from social cognitive models explain a substantial part of the variance in intention to consume and the actual dietary intake of saturated fat, fruit and vegetables, some studies have revealed that these determinants more strongly predict self-rated intake, that is, a rating of how much fat, fruit or vegetables people think they eat, than 'objectively' assessed intake (Brug et al., 1994; Lechner et al., 1997). Respondents who thought they consumed low fat or high fruit and vegetable diets generally had positive attitudes and high self-efficacy expectations towards consuming diets low in fat and high in fruit and vegetables (Brug et al., 1994; Lechner et al. 1997). In addition, self-rated intake was found to be a relatively strong predictor of the intention to change, whereas objectively assessed intake did not predict the intention to change in analyses including both self-rated and objectively assessed intake (Brug et al., 1994; Lechner et al., 1997). Self-rated intake was found to be inconsistent with objectively assessed intake. Most people in the Netherlands underestimate their fat intake or overestimate their fruit and vegetable intake (Brug \& Kok, 1995; Brug \& Lechner, 1995; Brug et al. 1994; Lechner et al., 1997). This underestimation or overestimation reflects a lack of awareness of people's own dietary intake. Since self-rated intake is predictive of the intention to change. it was concluded that lack of awareness is a major barrier for the intention to change to healthier diets. Only after elimination of this barrier can attitude, social influences and self-efficacy expectations towards a lower saturated fat and higher fruit and. vegetable intake become relevant (Brug \& Kok, 1995: Brug \& Lechner, 1995; Brug et al., 1994: Glanz et al., 1997: Lechner et al., 1997).

A factor that was found to be strongly associated with lack of awareness of personal dietary intake is 'comparison with others'. The less fat people thought they ate compared to peers of the same age and sex, the more they underestimated their own fat intake. Similarly, the more fruit and vegetables people thought they ate compared to peers of the same age and sex, the more they overestimated their fruit and vegetable intake. This comparison with others seemed to be optimistically biased, since most people thought they were eating less fat and more fruit and vegetables than their peers. It was concluded that an optimistic bias in the 
comparison with others may be an important determinant of the self-rated intake of fat, fruit and vegetables (Brug, Van Assema. \& Kok, 1998b).

\section{Stage models of behaviour change}

Stage models of behaviour change assume that changing behaviour is not a dichotomy, nor a gradual process along a continuum, but a process of proceeding through different stages in a predetermined sequence. Stages are distinct in that people in one stage differ from those in another stage in aspects like behaviour, cognitions or perceived barriers against proceeding to the next stage. Stage models imply that interventions aimed at behaviour change should be stage specific (Armitage \& Conner, 2000; Weinstein, Rothman, \& Sutton, 1998). The best-known stage models are the Transtheoretical Model (Prochaska, DiClemente, \& Norcross, 1992) and the Precaution Adoption Process Model (Weinstein, 1988; Weinstein \& Sandman. 1992: Weinstein \& Sandman, 2002).

The Transtheoretical Model (TTM) distinguishes five stages to maintenance of a new behaviour (Figure 1.2). In the precontemplation stage, people do not intend to change to a healthier behaviour. In relation to dietary behaviour, there is evidence that this may be because people are not aware that there is a need to change or because the expected negative consequences of a healthier diet (cons) outweigh the expected positive consequences (pros). In the contemplation stage, people intend to change within the next six months. In this stage, people consider changing to a healthier behaviour but are not yet acting. because the expected cons of the new behaviour still outweigh the pros. In the preparation stage, people intend to change within the next month. In this stage, people may be forming plans for changes or are already taking small steps towards actual change. In the action stage, people have actually changed to a healthier behaviour, but have not yet sustained this new behaviour for more than six months. In the maintenance stage, people have sustained their new, healthier behaviour for more than six months (Prochaska et al., 1992; Prochaska \& Velicer, 1997: Prochaska. Velicer, Rossi, Goldstein. Marcus. et al., 1994).

The Precaution Adoption Process Model (PAPM) distinguishes seven stages to the adoption of a precautionary behaviour (Figure 1.2). The unaware stage refers to a state in which people have never heard of a health issue or the association between a particular behaviour and its health consequences. In this stage, therefore. people will not have an opinion about the issue. For example, people may never have heard of the association between high saturated fat consumption and CVD risk. In the unengaged stage, people have heard about a health issue and may believe that it poses a major risk to others, but have not given much thought to their own personal risk status or to the necessity of taking action themselves. For example; people may know that a high saturated fat intake increases the risk of CVD. that many other people have high saturated fat intakes, but have never thought of the possibility that they may be engaging in the risk behaviour themselves. In the deciding about acting stage, people have become engaged in the issue, for example 

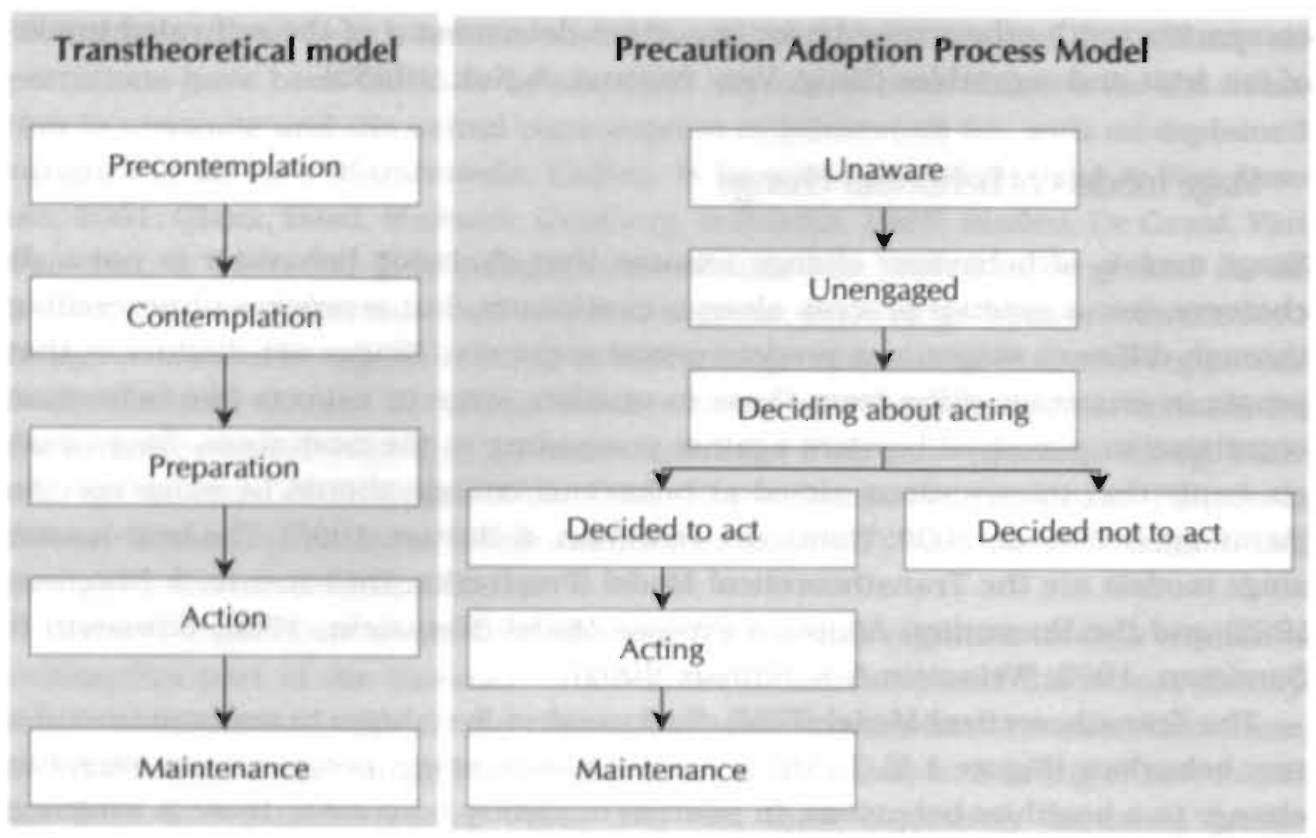

Figure 1.2. Stages of change according to the Transtheoretical Model and the Precaution Adoption Process Model (From: Weinstein \& Sandman, 2002)

acknowledging that they are engaging in risk behaviour for CVD themselves, and are considering whether or not to adopt a preventive behaviour. Factors that may determine the outcome of this stage include perceived personal susceptibility to the threat, severity of the threat, behaviour of others, beliefs about effectiveness of the precaution, costs, benefits and barriers. Perceived susceptibility (i.e., awareness of personal risk or risk behaviour) is particularly important in finally deciding to act. The deciding about acting stage can either lead to the decided not to act stage. which is a step out of the sequence towards acting. or to the decided to act stage, which is followed by the acting and maintenance stages (Weinstein, 1988: Weinstein \& Sandman, 1992; Weinstein \& Sandman, 2002).

An important difference between these two stage models is that the PAPM distinguishes several stages in the process prior to the deciding about acting stage. whereas the TTM defines this stage as one single stage, that of precontemplation. People who would be classified in the precontemplation stage according to the TTM can be in the unaware, unengaged or decided not to act stages according to the PAPM. The decided not to act stage is a significantly distinct stage, which is not acknowledged in the TTM. Since people in this stage have formed an opinion about an issue, they are more likely to be resistant to persuasion than people who have not formed an opinion, that is, those who are in the unaware stage. Furthermore, treating precontemplators as one group and providing stage tailored information to this group may mean that a considerable part of this group receive mismatched in- 
formation, particularly those who are not aware of being engaged in risk behaviour. Therefore, the more refined stage division in the early stages of the behavioural change process in the PAPM provides the opportunity to address people in these stages with more accurate stage tailored information.

An early version of the PAPM emphasised the role of awareness of personal risk behaviour more clearly (Weinstein, 1988). The model distinguished three phases to awareness of personal risk behaviour, viz., never heard of an issue (phase 1). knowing that many other people engage in risk behaviour (phase 2), acknowledging that one is engaged in risk behaviour personally (phase 3). Only after phase three has been reached could someone proceed through the further stages of change. These three phases to awareness, which are integrated in the unaware and unengaged stages in later versions of the PAPM, provide important theoretical input for the present thesis.

\section{Summarised findings of the determinant analysis}

Lack of awareness of personal dietary intake was identifled in the analysis of behavioural determinants as an important barrier for the intention to change. The PAPM also emphasises awareness of personal risk behaviour as a prerequisite for proceeding through the stages of change towards the decided to act and acting stages. Since awareness seems to be of great importance in changing dietary behaviour and since lack of awareness of the actual intake of saturated fat, fruit and vegetables is very common. priority should be given to developing interventions that address this barrier first. It is therefore important to identify effective intervention methods and strategies to raise awareness of personal dietary behaviour. An optimistic bias in comparing one's own dietary intake with that of others may be an important determinant to target.

\section{Intervention development}

Behaviour can be changed by influencing behavioural determinants. Therefore. after the phase of analysing behavioural determinants and selecting the most important and modifiable determinants, the next step is to develop interventions that address these important determinants. In this phase, theory-based methods for influencing the selected determinants have to be identified, translated into practical intervention strategies, delivered through a suitable channel that is able to reach the target population and evaluated for their efficacy.

\section{Theory-based methods for awareness raising}

\section{Feedback}

The PAPM proposes feedback as a strategy to increase awareness of personal risk behaviour (Weinstein, 1988). Feedback is a mechanism that functions in all 
complex systems, from the human body to organisational and technical systems. Feedback mechanisms function as self-regulatory systems and aim at maintaining homeostasis. A (negative) feedback loop consists of an input, a reference value or standard, a comparator and an output (Carver \& Scheier, 1998), In psychology and behavioural science, feedback is one of the mechanisms that are thought to regulate behaviour and influence behavioural determinants. The results of actions taken are fed back as input to the feedback system where they are compared with a reference, and the resulting output consists of the subsequent motivation and action. If there is a discrepancy between input and standard, motivation and behaviour are expected to change in such a way as to reduce this discrepancy (Carver $\&$ Scheier, 1998). This feedback process can function internally or externally and may be implicit or explicit (Bandura, 1997, p. 66). People can derive feedback from their own performances, or feedback can be provided by an external agent. Feedback information provided by an external agent provides people with information about some aspect(s) of their task performance, and is mostly aimed at improving behavioural performance (Kluger \& DeNisi, 1996).

Feedback about one's own risk behaviour (personal feedback) and feedback about the risk behaviour of peers (normative feedback) are types of feedback that are thought to contribute to increased awareness of personal risk behaviour. Feedback about people's own risk behaviour is thought to clarify their own status with regard to a risk behaviour, whereas feedback about the risk behaviour of peers is thought to reduce the optimistic bias in the estimation of their own risk behaviour compared to that of others. Increased awareness is thus expected to result in a motivation to improve performance and hence in progress through the stages to decided to act or having the intention to act. People in this stage can be induced to make the transition to acting by being provided with information about how to act, thereby emphasising that it is reasonably easy to act. in order to increase self-efficacy expectations (Weinstein \& Sandman, 2002).

\section{Social comparison information}

An optimistic bias in the comparison with others has been found to be associated with the lack of awareness of people's own risk behaviour. The PAPM regards an optimistic bias in the perception of people's own risk. compared to that of others as a barrier to awareness of personal risk behaviour (Weinstein. 1988). Since interpersonal or social comparison processes seem to play a role in the lack of awareness. theories of interpersonal processes may provide methods to increase awareness of dietary intake. Modelling and Social Comparison Theory both have interpersonal comparison processes as their core constructs and may therefore inform interventions aimed at increasing awareness.

\section{- Modelling}

Modelling. or observational learning, means learning by observing the behaviour of others, the role models. Through modelling complete behavioural sequences can be learned without going through a process of trial and error. Not 
only behaviour and behavioural skills can be learned through modelling, but attitudes and self-efficacy expectations can also be learned, strengthened or modified through modelling. Important prerequisites for modelling are attention for the role model and the modelled behaviour, and motivation to model the behaviour. Attention is expected to increase when the role model is similar to and attractive as a role model for the person who observes. Motivation is expected to increase when the behaviour of the role model results in positive consequences or rewards, for example feeling better about oneself or receiving social approval (Bandura, 1986, 1997). Modelling is widely used in everyday life, and can be induced in health education interventions by providing people with attractive role models that perform a desired behaviour or express the desired attitudes. Observational learning is expected to motivate people to try and perform the modelled behaviour themselves, or re-evaluate their attitudes and behaviour. In modelling, role models are typically presented as models that perform better than the persons that have to learn from the role model.

\section{- Social Comparison Theory}

According to Social Comparison Theory (Buunk \& Gibbons, 1997; Festinger, 1954: Suls \& Wheeler, 2000; Wood, 1989), people have a natural urge to evaluate their opinions, abilities and performances, preferably relative to an objective standard. If no objective standard is available, people will use less objective information, such as social comparison information, to evaluate their opinions, abilities and performances. Social comparison is most likely to occur with similar others, such as people of the same age and sex, with a similar lifestyle, but who are also similar on the dimension under comparison. For example, an amateur athlete will be more likely to compare his abilities with another amateur athlete than with a professional world-class athlete. Social comparison can serve several goals; self-improvement, aimed at improving a certain ability or performance; self-enhancement, aimed at feeling good about the self; or selfevaluation, aimed at getting accurate information about one's personal ability regarding a particular aspect. Three main directions of social comparison are distinguished: upward, that is, comparing with someone who is performing better on a particular aspect: downward, that is, comparing with someone who is performing worse on a particular aspect: and lateral, that is, comparing with someone who is performing equally well on a particular aspect. The direction of the social comparison made, depends on people's level of well-being and on the goal they seek to accomplish. According to Social Comparison Theory, positive role models (upward comparison) or negative role models (downward comparison) could both have an effect on motivation and subsequent behaviour.

Translating theoretical methods into deliverable interventions

\section{Interventions providing feedback}

Personal feedback can only be provided based on a particular input from a recipi- 
ent. Therefore, providing personal feedback information requires a strategy that makes it possible to assess information on personal behaviour or opinions first. Person-to-person communication between a counsellor (for example a general practitioner or a dietician) and a client is probably the best way to provide personal, assessment-based, information. Due to time and cost constraints, and the undesirable effect of making people dependent on health care, this may not be a feasible or desirable strategy to reach large groups of healthy people. Self-test forms and computer-tailored nutrition information are strategies that incorporate assessment-based feedback and can be distributed among large groups of people at relatively low cost and with limited time investment. These two strategies differ considerably in extensiveness, but both may have their advantages.

\section{- Self-tests}

Self-tests are simple tests that can be easily performed by an individual and that give a rough indication of someone's status related to a particular topic. Medical self-tests are quite common, with examples including using a thermometer for taking temperature. blood sugar tests for diabetics or pregnancy tests. There are also multiple self-tests avallable in the area of everyday behaviour. Many popular magazines include tests allowing people to assess aspects like, for example, their emotional intelligence or risk-taking behaviour by answering a few questions. Even though these self-tests are mainly used for entertainment. they still may have the capacity to classify people into certain categories. Selftests like these can also be developed for health behaviour, including dietary behaviour. Based on a few questions, a person can be classified into a category in terms of intake, which corresponds with feedback that is relevant to people in that category.

The specificity of the feedback that can be provided by these self-tests is rather basic, but more person-specific than generic "one size fits all' information. An advantage of the more basic nature is that the self-tests are short and easy to administer and do not require extensive reading, which may appeal to some population groups. Moreover, self-tests are easy to distribute among the population by health education professionals. Such brief, low-intensity interventions may probably not have a strong effect on the outcome of interest, but may. because of their broad reach, have a great overall impact (Abrams, Emmons, \& Linnan, 1997) and are therefore worth being explored for their efficacy.

\section{- Computer tailoring}

Computer tailoring is a strategy that makes it possible to provide large groups of people with individually tailored health information. Computer tailoring combines characteristics of person-to-person counselling with mass media education. Computer-tailored information is by its very nature assessment-based. A computerised process allows information to be attuned to the assessed characteristics of an individual, such as behaviour, opinions about that behaviour and motivation to change. This procedure results in the provision of information 
that is relevant to one particular person and not to others (De Vries \& Brug. 1999: Dijkstra \& De Vries, 1999: Kreuter, Farrell, Olevitch, \& Brennan, 2000). The assessment-based nature, the personal approach and the potentially wide reach make computer tailoring a suitable instrument for providing personal feedback on dietary intake levels to large groups of people. Furthermore, providing individualised and personally relevant information attracts more attention to the message, reduces the amount of information to be read and leads to more active involvement and cognitive processing of the information. These features are all expected to result in an increase in persuasive power of the information, and therefore in greater effects (McGuire, 1985; Petty, Barden, \& Wheeler. 2002).

Computer tailoring has become possible in the last decade through improved computer technology. A number of studies have been conducted to test the efficacy of computer-tailored health education interventions, and a variety of health behaviours have been addressed with computer-tailored interventions. such as smoking cessation (Dijkstra, De Vries, \& Roljackers, 1999; Strecher, 1999), increasing physical activity (Bock, Marcus, Pinto, \& Forsyth, 2001: Bull. Kreuter, \& Scharff, 1999), healthy diet promotion (e.g.. Brug. Campbell, \& Van Assema, 1999; De Bourdeaudhuij, Brug. Vandelanotte, \& Van Oost, 2002), weight loss (Kreuter, Bull, Clark, \& Oswald. 1999), participation in breast cancer screening (Rakowski, Ehrich, Goldstein, Rimer, Pearlman, et al., 1998; Skinner, Strecher, \& Hospers, 1994), participation in colorectal screening (James. Campbell, \& Hudson, 2002), encouraging early cancer detection behaviours (De Nooijer, Lechner, Candel, \& De Vries, 2002) and prevention of osteoporosis (Blalock, 2002). A variety of theoretical backgrounds have been applied, such as the Health Belief Model, the Social Cognitive Theory, the Theory of Planned behaviour, the ASE model, the Transtheoretical Model and the Precaution Adoption Process Model. The tailored interventions, and especially tailored nutrition interventions, were generally found to be more effective in inducing the desired outcomes at the behavioural or determinant level than non-tailored information or no information. Several reviews of computer-tailored interventions have illustrated this, either related to interventions targeting various behaviours (Ryan \& Lauver, 2002; Skinner, Campbell, Rimer, Curry, \& Prochaska, 1999; Strecher, 1999) or for computer-tailored nutrition interventions in particular (Brug et al., 1999; Brug. Oenema, \& Campbell, 2003).

\section{- Challenges for computer tailoring research}

The evidence for the effectiveness of computer-tailored (nutrition) information is largely based on the results of studies using printed tailored information. such as tailored letters, newsletters or magazines. New challenges in computer tailoring research include tailored information provided through 'new' media like telephone, video, audio, CD-ROM, the computer, e-mail, or the Internet (Abrams, Mills, \& Bulger, 1999; De Vries \& Brug. 1999: Dijkstra \& De Vries. 1999; Kreuter et al, 2000). Furthermore, even though several suggestions, 
derived from theory and some empirical evidence, have been made as to the reasons why tailoring exerts a greater effect than generic information, the working mechanisms of computer tafloring are still largely unknown or at least unproven. Gaining further insight into the working mechanisms and essential elements of computer talloring may provide the opportunity to enhance the efficacy of computer-tailored information (Abrams et al. 1999: De Vries \& Brug. 1999: Kreuter et al., 1999; Skinner et al., 1999).

Although computer-taflored nutrition interventions using new media, such as telephone, multi-media, computer or the Internet, are now becoming increasingly available (e.g., Anderson, Winett, Wojcik, Winett, \& Bowden, 2001; Block, Miller, Harnack, Kayman, Mandel, \& Cristofar, 2000; Campbell, HonessMorreale, Farrell, Carbone, \& Brasure, 1999: Delichatsios, Friedman, Glanz, Tennstedt, Smigelski, et al., 2001), few of such interventions have been evaluated. Therefore, there is a need for studies that evaluate the effects of these more advanced applications of computer tailoring.

\section{- The Internet as a new channel for delivering computer-tailored health information}

The Internet has become a widely used channel of communication and an important source of information, including health information. Health information is among the topics most searched on the Internet (Raad voor de Volksgezondheid en Zorg. 2000). Access to the Internet is rapidly growing. In 1998 only $14 \%$ of the households in the Netherlands had a personal computer (PC) with. Internet access, while $49 \%$ did not have a PC at home. In 2002, 55\% of the households had a PC with Internet access, while only $31 \%$ did not have a PC at home (Centraal Bureau voor de Statistiek, 2004).

This wide use of and interest in health information through the Internet and the large and stil! growing access to the Internet make it an interesting medium for providing computer-tailored health information. Some of the advantages and disadvantages of the Internet as a medium for delivering computer-tailored interventions are discussed in this section.

The Internet, and particularly the World Wide Web, has been proposed by many authors as a promising medium for delivering computer-tailored health education (De Vries \& Brug. 1999; Dijkstra \& De Vries, 1999: Kreuter et al. 2000). The computer-based nature of the computer tailoring technique makes it particularly suitable for interactive use directly on a computer or through the Internet. Using an interactive medium for delivering tailored health education. may more fully utilise the potential of computer tailoring. which may enhance the overall impact of computer-tailored interventions. Interactivity is one of the features of computer-tailored information that can be fully utilised in tailored interventions provided through interactive channels. Interactivity requires active involvement with the information. which is thought to induce more cognitive processing of the information (Petty \& Cacioppo, 1986: Strecher, Greenwood. Wang. \& Dumont, 1999). Furthermore, using an interactive medium makes it 
possible to decrease the time span between assessment and feedback delivery. increasing the likelihood that the feedback is indeed accurately tailored to the person's assessment results at that time. Computer-tailored information can be made to resemble interpersonal counselling even more by including elements of social support, which may increase its efficacy. Interactive media provide the opportunity not only to tailor the information. but also to tailor the necessary assessment questions to an individual. Irrelevant questions can be skipped and respondents with a greater interest in information can go to a deeper level of assessment and tailored advice.

Numerous other advantages of using the Internet as a medium for delivering tailored information may enhance its efficacy. The Internet is perceived as more anonymous than other media (Cline \& Haynes, 2001: Strecher et al.. 1999). which may result in less socially desirable answering, thus making it possible to provide more accurately tailored information. 'Noise' between information provider and receiver may be reduced by using sophisticated interactive media (Abrams et al., 1999).

Efficiency may also be increased by using interactive media. Once created, a tailored programme provided through the Internet or other interactive medium does not require any handling by intermediaries or use of materials, thereby reducing the costs of delivering the tailored information (Brug et al., 2003). For example, tailored information provided through a web-site on the World Wide Web only needs to be hosted and updated periodically, while a print-delivered version of computer-tailored materials would require distributing assessment tools, importing the returned assessment tools into a database, printing the tailored information and sending it to a recipient. Delivering computer-tailored information through the World Wide Web potentially allows large groups of people to be reached at relatively low costs, at any point in time, all over the world, as long as there is a computer online.

The advantages for the recipients of Internet delivered taflored information may also be manifold. It provides an opportunity to get a quick impression of personal status or performance on health or health behaviour, through a medium, that is perceived as anonymous, and is accessible at any time. Communication is quick, while the pace at which people work their way through the information can be self-determined. On the recipient side, however, there may also be many disadvantages. Whereas the costs in terms of time, effort and resources for the provider may be low, using a computer-tailored programme provided through the Internet may require increased effort and skills of the recipient. The recipient has to start a computer, get online, find the web-site. decide whether this web-site comes from a credible source, find out how to get the tailored information. stay on the site for a sufficient length of time to complete the assessment and read the feedback, use interactive elements, resist the temptation of surfing to other sites and deal with possible technical problems.

The Internet may also incorporate barriers towards effective persuasive communication. New noise may be introduced between the provider and the 
recipient. For example, the appearance of the information provided through the Internet depends on the speed and capacity of the recipient's computer, or users may not proceed through the information as intended by the provider. Even though interactivity may lead to greater involvement with the message, the Internet is also a fleeting medium, used to quickly find and scan information and then move on to other pages. Reading information from the screen may be more difficult and result in less thorough reading than printed information (Murphy, Long. Holleran. \& Esterly. 2003). Apart from searching for 'primary information', people also use the Internet for 'second opinions' about a particular topic. This search for 'second opinions' may in some cases lead to sites that provide opposing information, for example sites that advertise high fat products, whereas the tailored information advocated low fat products. Furthermore, searching many sites may lead to information overload (Cline \& Haynes, 2001). The advantage that tailored information is limited to personally relevant information only, thus limiting the amount of information provided, may be eliminated by this information overload in the Internet environment. Furthermore, the Internet may be primarily perceived as a source of gathering information about health, and not as a source for being advised on how to improve health (Cline \& Haynes, 2001). This information gathering may in itself be a form of tailoring ('self-tailoring'), making expert-system guided tailored information programmes redundant. Finally, not everybody can be reached with information provided through the Internet (Cline \& Haynes, 2001: Eng, Maxfield. Patrick. Deering. Ratzan, \& Gustafson, 1998).

Given the challenge of evaluating the effects of more advanced applications of computer-tallored interventions and the potential advantages of the Internet as a channel for delivering the information to the target population, the research presented in this thesis explored the possibilities of a computer-tailored nutrition intervention that is web-based and ready for distribution through the Internet. However, instead of distributing this intervention through the actual Internet, it was tested in more controlled situations.

\section{Interventions based on principles from social comparison theory and modelling}

Although there are several methods for providing social comparison information. little is known about ways of effectively providing social comparison information related to dietary intake and promoting awareness of personal dietary intake. Role models or comparison persons are required in interventions providing social comparison information. Role model information can appear in many different ways, such as face-to-face contact with real life models, role models presented through video or audio channels, fictitious persons, interactive contact with a role model through the Internet or printed role model stories. Furthermore, role models or comparison persons can perform better, equally well or worse than the person who observes. Since positive and negative role models can have an effect on the motivation and intention to change, it was decided to provide social comparison information in the form of printed positive and negative role model stories. 


\section{Intervention evaluation}

Evaluation is an integral part of all phases in the process of planned intervention

development (Figure 1.1). Various levels of evaluation can be distinguished including evaluation at the outcome level, at the impact level and at the process level (Green \& Kreuter, 1999). The ultimate goal of an intervention is to induce changes at the outcome level, that is, improved health and quality of life. However. it is seldom possible to evaluate effects of primary prevention interventions for behavfour change at the level of health outcomes and quality of life, especially not for chronic diseases like CVD and cancer. Moreover, it may be inefficient to evaluate interventions at this level, since. if interventions are indeed developed following the planned health education approach, and thus grounded in a proper epidemiological analysis of the relation between behaviour and health, behaviour change will eventually result in changes in health and quality of life. Since behaviour change is detectable at much shorter notice. it is more efficient to evaluate interventions thoroughly at the level of behaviour. that is, the impact level. Moreover, behaviour change can only be expected if the intervention enables changes in the behavioural determinants. Therefore, evaluation at the level of determinants, that is, the intermediary impact level, is also important.

\section{A phased approach to effect evaluation}

The choice of the level of evaluation and the evaluation approach also depends on the phase of intervention development. A phased approach to efficacy evaluation has been recommended (Campbell, Fitzpatrick. Haines, Kinmonth, Sandercock, et al., 2000; Rossi. Freeman, \& Lipsey, 1999). Such a phased approach proceeds from small-scale evaluation in a highly controlled situation in the early phases of intervention development, through larger-scale evaluation in more real life and less controlled settings, to large-scale implementation research. Ideally, it is only after efficacy in one phase has been established that the intervention should be subjected to evaluation in the next phase. An important question in the first phase is whether the intervention is able to produce an effect in a controlled setting. under optimal conditions. After the intervention has proved to be able to do this, the next phase is to evaluate whether the intervention effects are also found under less optimal, more real-life situations. If in this phase the intervention has proved effective in producing the intended effect, the intervention is suitable for large-scale implementation, or integration into multi-component interventions. The third phase in the phased approach to intervention evaluation would be concerned with effectiveness of the intervention, that is, whether the intervention is able to produce the expected health outcome.

\section{Process evaluation}

Effect evaluation should preferably be accompanied by process evaluation. Proc- 
ess evaluation aims to determine to what extent the intervention was actually implemented, whether it was implemented as intended, whether the recipients were actually exposed to the intervention, whether recipients used the intervention as intended and whether the intervention elements and contents were appreciated, comprehended and accepted by the recipients. The process evaluation adds to the degree of certainty with which the effects found in the effect evaluation can be ascribed to the intervention. If no effects of the intervention were found, information from the process evaluation could help to determine whether this lack of effect was due to less than optimal implementation, lack of exposure to the intervention, or less than optimal appreciation. comprehension and acceptation of the intervention's contents. The process evaluation may provide suggestions for improvements to the intervention and may indicate how improvements could be made.

\section{Intervention implementation and dissemination}

Interventions that have proved to be efficacious in inducing the desired changes in determinants and behaviour can only have an impact on health and quality of life in a population when a substantial proportion of the target population is exposed to them. Careful implementation and dissemination of an efficacious intervention is therefore a separate step in the process of planned health education. This step is, however, beyond the scope of this thesis.

\section{Outline of the thesis}

The studies presented in this thesis describe the efficacy and process evaluation of the interventions that were developed following the model of planned health education. Two interventions were studied, both using feedback as their main change strategy: a web-based computer-tailored nutrition education programme and printed self-test forms. In addition, we explored an as yet experimental intervention using social comparison information as a strategy for change, in the form of printed role model stories.

Chapter iwo provides an overview of the history. the rationale and the technique of computer tailoring, reviews the effects of computer-tailored nutrition education and points out future directions of computer-tailored nutrition education.

Chapters three and four present first-phase evaluations of the feedback interventions. The primary aim was to assess the possible efficacy of the interventions in a controlled setting. Post-test measurement was immediately after exposure to the intervention and assessed effects on determinants and process measures. The studies described in Chapters three and four use the same data set. Chapter three evaluates the effects of the web-based tailored nutrition intervention, while chapter four reports on the effects of the web-based tailored intervention and the self-test forms. 
Chapters five and six describe the studies that were conducted to establish the effects of the tailored intervention on the short and longer term in less controlled and more real life settings. The data for these studies were collected in one field study, but the results are described in separate chapters. Chapter five describes the effects of the tailored intervention at three weeks post intervention. This study included a more extensive process evaluation. Process measures were used to establish by which processes computer-tailored interventions may exert their effects. Chapter six describes the effects of the web-based tailored nutrition education intervention six months post intervention.

Chapters seven and eight are concerned with social comparison information as a possible method to induce awareness, intention to change and dietary change, Chapter seven reports on an exploratory study into the occurrence and nature of interpersonal comparison in dietary fat intake, while chapter eight presents a preliminary evaluation of the effects of positive and negative role model information on awareness, motivation and intention to change fruit intake.

Chapter nine summarises and integrates the results of the studies, discusses methodological and intervention related issues and provides some implications for practice and suggestions for further research. 


$$
\text { - }
$$




\title{
The use and impact of computer-tailored nutrition education
}

\begin{abstract}
Computer-tailored nutrition education is an innovative and promising tool to motivate people to make healthy dietary changes. It provides respondents with individualised feedback about their dietary behaviours, motivations, attitudes, norms and skills, and mimics the process of 'person-to-person' dietary counselling. The available evidence indicates that computer-tailored nutrition education is more effective in motivating people to make dietary changes than general nutrition information, especially for reduction of dietary fat. The effectiveness of computer tailoring has been attributed to the fact that individualised feedback commands greater attention, is processed more intensively, contains less redundant information, and is appreciated better than more general intervention materials. Interactive technology, such as the Internet and the World Wide Web, offers good opportunities for the application of computer-tailored nutrition education, and a first controlled study of web-based computer tailoring shows promising results. However, using the web for interactive personalised nutrition education also presents new challenges.
\end{abstract}

Published with minor modifications as: Brug, J., Oenema, A., \& Campbell, M. (2003). Past, present, and future of computer-tailored nutrition education. American Journal of Clinical Nutrition, 77, 1028S-1034S. 


\section{Introduction}

Computer tailoring is a health education technique that has become popular in dietary change research in the past decade, and is now in the process of being adopted by nutrition educators (De Vries \& Brug, 1999; Skinner et al., 1999). This is not surprising, since computer-tailored interventions have been applied and studied in relation to several health behaviours, and were generally found to be more effective than their non-tailored equivalents (Skinner et al., 1999), especially in promoting healthy dietary habits (Brug et al., 1999). Computer-tailored interventions mimic, to a certain extent, a classic tailoring technique, that of 'person-to-person' counselling. As in counselling, computer-tailored interventions provide people with information that is based on their individual characteristics, e.g., their behaviour. attitudes and perceived barriers, which makes the information personally relevant. In computer-tailored interventions, the diagnostic and educational expertise and techniques of the counsellor are documented in a computerized expert system. The present paper first describes the rationale for the application of computer tailoring in nutrition education by reviewing how the steps of carefully planned health education can be applied to computer tailoring. The paper then proceeds with a short outline of what is necessary for computer-tailored nutrition education and how computer-tailored interventions are developed and implemented. It further presents the available evidence for the effectiveness of computer-tailored nutrition education and describes the results of the few studies that have to date investigated why and for whom computer-tailored interventions may be effective. The final sections of the paper discuss the future of computer tailoring in the era of the Internet and the World Wide Web, and present some preliminary results from a study evaluating a web-based computer-tailored nutrition intervention.

\section{Why should we use computer tailoring?}

It is especially since the publication of Green \& Kreuter's Precede model and its successor Precede-Proceed (Green \& Kreuter, 1999) as well as other, similar planning models (McKenzie \& Smeltzer, 2001), that health educators have recognized the importance of careful theory-based intervention planning. A schematic representation of such planning models is depicted in Figure 1.1 (see p. 2). According to these planning models, the first step in health promotion planning is the identification of a health problem that is serious and prevalent enough to justify spending time, money and other resources on developing and implementing an intervention. In the second step, the specific behavioural and environmental risk factors for the health problem of interest should be identified, as well as who are exposed to these risk factors.

The third step in planned health promotion is to investigate the psychosocial and environmental determinants of exposure to risk factors. This planning phase should identify as precisely as possible why people in the target population engage 
in risk behaviour. In relation to a nutrition behaviour, the determinant analysis should for example point out why people eat too much saturated fat and whether these determinants differ in relation to such factors as gender, age and education. This phase in the identification of the determinants of engaging in risk behaviours has long been disregarded. For a long time, nutrition educators assumed, and some still do, that it is enough to know what the problem is and that diet contributes to this problem. Merely telling people that their diet is putting them at risk was supposed to make people change their food habits. Nowadays, however, a large body of empirical evidence shows that health beliefs are only one possible determinant of nutritional habits (Brug \& Van Assema, 2001), and often not the most important determinant. It is therefore necessary to conduct a more thorough inventory of behavioural determinants as part of the development of planned nutrition education. Five categories of important as well as changeable proximal determinants have been identified (Armitage \& Conner, 2000): intentions or motivations, a weighing of pros and cons (attitudes), social influences, perceived behaviour control and personal norms (Figure 2.1).

These categories of determinants explain up to $50 \%$ of the variance in fat intake (Baranowski, Cullen, \& Baranowski, 1999; Stafleu et al., 1991). Factors such as sex, age or socio-economic status are regarded as more distal correlates of dietary habits. Differences in eating habits between, for example, men and women are supposed to be a result of sex differences in the more proximal determinants (Flay \& Petriatis, 1994). The insight that a limited number of determinant categories predict dietary habits relatively well does not mean that determinant research is no longer necessary (Bartholomew, Parcel, Kok, \& Gottlieb, 2001). The determinants should be investigated separately for each specific dietary behaviour, and for each population (or population segment), in order to detect which determinants are

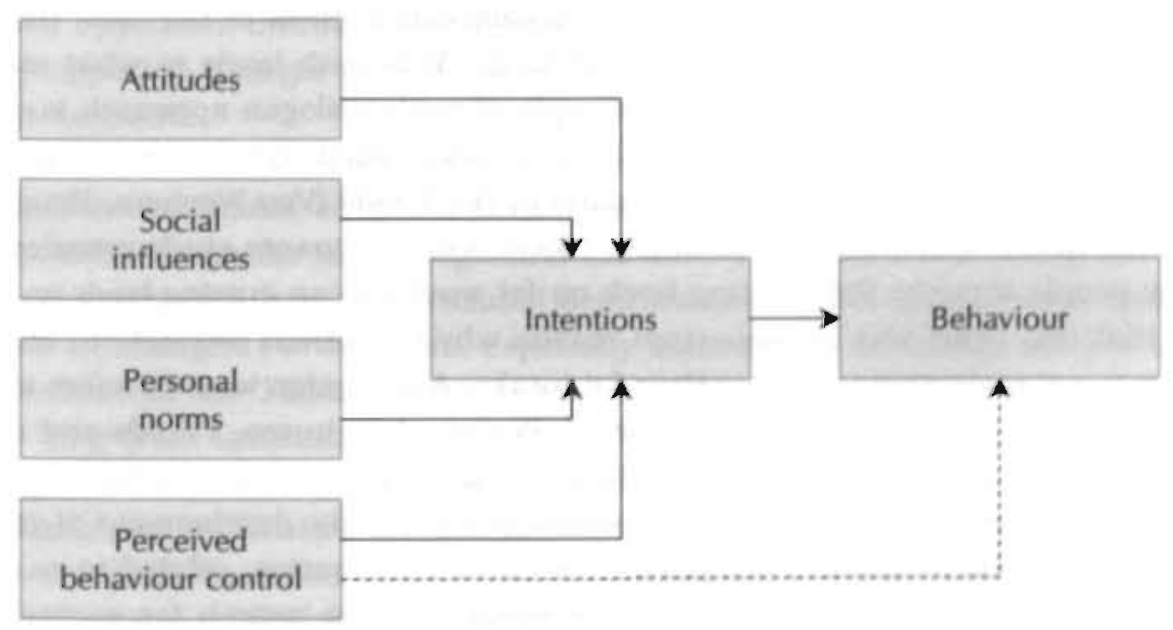

Figure 2.1. Psychosocial determinants of health behaviour (From: Armitage \& Conner, 2000) 
most important in a specific context as well as to identify the specific beliefs that underlie the determinants. For example, if a weighing of pros and cons (resulting in a positive or negative attitude) has been identified as the major determinant of motivation to eat a low-fat diet among younger women, this does not mean that the same applies to older men. Social influences may be more important in this group. And if attitudes are identified as the main determinant, it is still necessary to find out which specific positive or negative beliefs should be addressed to change these attitudes. It may well be that beliefs about health consequences are important for attitude formation among relatively wealthy people, but that beliefs about costs are more important for attitudes toward eating less fat among people who are less well off.

\section{Intervention development: one size fits all?}

In the next planning phase, health education methodologies and intervention techniques should be selected and translated into specific intervention activities in order to address the relevant determinants that can mediate the targeted behaviour changes. Since diet-related health risks, such as risk for cardiovascular disease and diabetes, are prevalent among many population segments, many people need to be addressed with interventions aimed at prevention. Therefore, mass media campaigns aimed at whole populations have often been applied to encourage people to adopt healthier diets. Traditionally, mass media campaigns have made use of generic health education materials and messages to address the target population. If the campaign is carefully planned, these generic materials are fitted as closely as possible to the behavioural and environmental determinants of the risk factors targeted by the campaign. However, as we have argued, these determinants differ between people. Since generic materials are supposed to appeal to large groups of people, generic health education can, generaily speaking. follow two paths. The first is to address just one or very few determinants (beliefs or barriers) that are generally important for the population at large. This path leads to what may be called 'slogan interventions'. A good example of such a slogan approach is one of the national campaigns to encourage fat reduction, called "Let Op Vet" (Watch the Fat), that was mounted in the Netherlands in the 1990s (Van Wechem. Brug. Van Assema. Kistemaker, Riedstra. \& Löwik. 1998). A determinants study revealed that many people thought that cutting back on fat would mean cutting back on taste and that this belief was an important reason why the Dutch population, on aver= age, was not motivated to reduce their fat intake. A campaign was therefore specifically designed to tackle this specific belief. Posters, brochures. TV-ads and recipe booklets were used to communicate the message that a diet low in fat can be at least as tasty as, a high fat diet. The second approach to the development of generic health education materials is to provide as much information, related to as many potentially important determinants, as possible. In this 'search for yourself approach, it is not assumed that all people have the same information needs. Rather, it is assumed that people will read and sift through. often extensive, collections of 
potentially relevant information. They are supposed to select the information that is relevant to their personal situation, in relation to their specific risk behaviour. motivations and beliefs. It has been found, however, that only people who are already motivated to cut back on fat are willing to search through lengthy brochures on dietary fat reduction for information that applies to their personal situation.

Both approaches have obvious limitations in their ability to significantly motivate risk behaviour change. The 'slogan approach' may lead to significant changes in the specific belief that is addressed among a proportion of the people who have that belief, but there is little chance that changing this belief will be more than a first step toward behaviour change. The 'search for your self approach will at best have an impact on motivated people, whereas many people are not motivated to (further) reduce their intake of saturated fat (Brug. Hospers, \& Kok. 1997; Glanz et al. 1997).

\section{'Ready-to-wear' health education}

Various attempts have been made in the past to match the content of health education more closely with the needs of the target populations. Most of these attempts can be characterized as target group segmentation, based on principles of social marketing. In target group segmentation, different sub-populations are distinguished that are more homogeneous in their information needs than the population at large. Target group segmentation is most often based on socio-demographic characteristics. For example, men may generally have different beliefs about fat reduction than women. Therefore, it may be worthwhile to produce sex-specific fat reduction brochures. Similarly, preferences for the specific language, examples or illustrations used in health education material may differ, for example according to age, which may lead to different materials for different age groups. Within each target group segment, it is still possible to use the 'slogan' or the 'search for yourself approach. but because the intervention is targeted in terms of sex or age. it may appeal to a larger proportion of the specific segment or the searching may be less extensive.

However, as we have argued, socio-demographic variables are not direct or proximal determinants of health behaviour. Useful target group segmentation is only possible if specific target segments are indeed homogeneous enough in their psychosocial beliefs (i.e., the proximal determinants of eating behaviour and motivation to change). Furthermore, especially since stages-of-change models came into fashion in the 1980 s, health behaviour change is no longer regarded as a single step from unhealthy (e.g., eating a diet high in saturated. fat) to healthy (eating a diet low in saturated fat) behaviour. The stages-of-change concept from Prochaska and DiClemente's Transtheoretical model (Brug et al., 1997; Prochaska \& Velicer, 1997) and Weinstein's Precaution Adoption Process model (Weinstein et al. 1998) postulate that behaviour change is a phased process. These models state that interventions should be stage-specific because different determinants are important for each stage transition. As a consequence, people in different phases 
in the behavioural change process need different information, skills training, environmental changes, and so on to proceed to the next stage. In fact, the stages of change may serve as segmenting variables for clusters of psychosocial characteristics (Van Duyn et al., 2001). It may therefore be both possible and more effective to segment health education audiences based on these proximal determinants and on motivational stage of change instead of, or in addition to, socio-demographic characteristics.

\section{Tailored nutrition education}

Tailored health education can be regarded as the ultimate aim of target group segmentation: it brings in individualisation and personalisation of health education based on socio-demographic, behavioural, motivational and other psychosocial as well as physical characteristics (De Vries \& Brug. 1999; Kreuter et al., 2000). This concept, which is also being used in the field of product marketing, has been called 'mass customisation' and 'relational marketing.' It has been made possible in recent years by the ability of companies to produce and tailor advertising based on vast amounts of data regarding customer demographics, preferences and buying habits.

In health education, talloring has been defined as "any combination of information or change strategies intended to reach one specific person, based on characteristics that are unique to that person. related to the outcome of interest. and have been derived from an individual assessment" (Kreuter et al., 2000; Kreuter \& Skinner, 2000). If we translate this general definition into nutrition education practice, a tailored nutrition education intervention would be:

- a combination of nutrition information or dietary change sirategies:

- aimed at a specific person:

- based on this person's dietary habits and/or stage of change and the determinants of these habits:

- assessed for this person in particular.

This is of course what many nutrition counsellors do in everyday practice. However, personal counselling is too time consuming and therefore too expensive to apply for each and every individual who, for example, has a diet high in saturated fat or low in fruit and vegetables. Nowadays it is possible to apply tailoring by using interactive technology. which makes personalisation of nutrition education applicable to large groups of people at relatively low costs. This is what is referred to as computer tailoring. In computer tailoring, the nutritional and educational expertise of a nutrition counsellor, or even better, the combined expertise of many counsellors, is translated to a series of 'if-then' statements and as such documented in an expert system. After a computer-tailored nutrition education system has been developed, it can be applied and distributed relatively independently of nutrition education expertise. In other words: computer-tailored systems make distribution of expert advice possible for non-expert intermediaries, or without any intermediaries. 


\section{How is computer tailoring done?}

The process of computer talloring attempts to mimic the process of personal counselling: people are surveyed or interviewed and the results are used to develop individualised feedback and advice. In the computer-tailored interventions developed to date, the survey is generally self-administered or administered by telephone and the survey results are keyed or automatically scanned into a data file. The tailoring expert system analyses these data and links them with a feedback and advice source. This feedback source is a message library or archive that contains appropriate feedback and advice for each survey response.

The survey is to provide the 'diagnosis' for the individual feedback and advice. and should therefore be aimed at assessment of the variables that are important for inducing dietary change, an assessment for which only valid and reliable measurement tools should be used (see Dijkstra \& De Vries, 1999 and Kreuter et al., 2000 for excellent descriptions of the process of generating tailored health education materials).

\section{Does computer-tailored nutrition education work?}

Just as it is not possible to conclude in general that health education leaflets, brochures or videos are effective or ineffective, it may be an illusion to expect that generalised conclusions can be drawn about the effectiveness of computer tailoring. However, several recent reviews have demonstrated that tailored printed materials generally outperform standard health education messages (Brug et al., 1999; Skinner et al. 1999). Brug and colleagues reviewed the literature on the impact of computer-tailored nutrition education specifically for dietary change, and concluded that tailored nutrition education is more effective than general nutrition education. especially for fat reduction (Brug et al., 1999). An additional analysis of the pooled results of three trials of computer-tailored feedback in the Netherlands showed that computer-tailored interventions resulted in a $5.4 \%$ lower fat intake, compared to a $1.4 \%$ drop in a general nutrition information control group approximately four weeks after the intervention (Brug. 1999).

\section{Why should computer-tailored nutrition education be more effective?}

The reason why computer tailoring should offer better prospects for effective intervention than general nutrition information has not been fully explored yet. How ever, the available studies as well as more general behaviour change models and theories allow possible working mechanisms to be identified: personalisation of the nutrition education, better exposure to and more intensive cognitive processing of the educational information, greater personal relevance of the messages, and the 
self-evaluation properties of tailored feedback (Brug et al., 1999: Dijkstra \& De Vries, 1999; Kreuter et al., 2000).

It has been argued that the personalisation of the feedback itself, i.e., putting the recipient's name on the feedback (similar to direct mail), determines the effect of tailoring. Although this may indeed be part of the story, various studies have shown that individually tailored interventions have greater effect than merely personalised messages (Brug. Glanz, Van Assema, Kok, \& Van Breukelen, 1998a; Brug. Steenhuis, Van Assema, \& De Vries, 1996: Kreuter et al., 2000) indicating. that talloring is more than just personalisation.

The first prerequisite of an intervention to have any impact is that the target population is exposed to it (McGuire, 1985). There is ample proof that computertailored information leads to better exposure. Computer-tailored feedback is more often read in its entirety, is read more thoroughly, and is more often stored as well as discussed with others than general nutrition information (Brug et al. 1998a; Brug et al., 1996; Kreuter et al., 2000; Skinner et al., 1999). A second prerequisite for nutrition education to have an effect is that the information is understood and cognitively processed. The fact that computer-tailored feedback is more often discussed with others may be a first indication that computer tailoring leads to more intensive cognitive processing of the information (Brug et al., 1999). Furthermore, because only personally relevant information is included in the tailored messages. computer-tailored nutrition education will contain less extraneous information. People may therefore restrict their information-processing capacity to information that is relevant to them. Kreuter and colleagues have found evidence that computer-tailored information is indeed processed more intensively (Kreuter et al. 1999). In a randomised trial they studied differences in the quantity and quality of people's cognitive responses to tailored and non-tailored weight-loss information. The results showed that computer tailoring led to more positive thoughts. more personally relevant thoughts stronger motivational thoughts and more self-assessment thoughts related to weight and weight-loss behaviours. Petty and Cacioppo's Elaboration Likelihoodl Model (Petty \& Cacioppo. 1986a) asserts that health information may be processed via two alternative routes: central or peripheral. The central route, requiring more cognitive processing and leading to more elaborations, is supposed to be stronger when the information is regarded as personally relevant. Further, central route processing will lead more readily to lasting attitude changes. The study by Kreuter et al. (1999) suggests that computer-tailored information is more likely to be processed centrally.

In a comprehensive review of the effectiveness of nutrition education. Contento and colleagues concluded that nutrition education was more likely to lead to healthier diets if more of the following prerequisites were met (Contento, Balch, Bronner, Lytle, Maloney, et al., 1995):

- Nutrition education should be tailored to motivators and reinforcers that are personally relevant to the people in the target group.

- Nutrition education should apply personalised self-evaluation and self-assessment techniques. 
- People in the target group should be able to participate actively in the nutrition education intervention.

As stated above, computer tailoring enables the application of these important health education methodologies and techniques to dietary behaviour change. It has indeed been established that computer-tailored nutrition education is perceived to be more personally relevant and that perceptions of personal relevance are positively associated with the impact of computer tailoring (Brug et al., 1999: Kreuter et al., 2000). Self-evaluation may be especially important in nutrition education. Many people are not aware of how inadequate (from a health promotion point of view) their personal dietary habits are (Brug et al., 1997: Lechner, Brug, De Vries, Van Assema, \& Mudde, 1998). For example, it has been repeatedly found that many people with diets that are higher in fat than is recommended by health authorities are not aware of their high fat diets. They believe that their diets are low in fat (Brug \& Van Assema, 2001; Brug et al., 1994: Glanz et al., 1997). This lack of awareness results in a lack of 'need to change' and subsequently, in a lack of motivation to reduce fat consumption ('why change if nothing is wrong?'). The fact that people have no clear idea of how much fat they eat is not surprising given that fat intake is a very complex behaviour, involving consumption of various food items in different combinations that may be prepared in different ways. Expert nutritional knowledge and advanced cognitive skills are needed to be able to assess and evaluate personal fat intake, even more so for different types of fat (e.g., saturated, monounsaturated). Computer-tailored systems can provide such objective feedback by incorporating the relevant expert knowledge and performing the calculations necessary to determine fat intake and compare it with recommendations.

People have a tendency to evaluate their own behaviour by comparing themselves with others (Buunk \& Gibbons, 1997). Most often, they do this in a 'selfserving manner' that is, they tend to compare themselves with people who they perceive to perform worse. This often results in a so-called optimistic bias, in that most people think that they perform better than most others (Weinstein. 1980). This is also true with regard to fat intake: many people seem to evaluate their fat intake not in comparison to dietary recommendations but by comparing their diet with what they think other people eat. In most cases, these 'others' are not a realistic representation of their peer group but a 'prototypical' group of high fat eaters. A majority of people therefore believe that their diet contains less fat than that of 'others'. Even though people may think that their fat intake does not meet the nutritional standards, as long as they believe that they eat less fat than most others, they may still not perceive a need to change. Computer-tailored feedback. however, provides the opportunity to give people feedback about their consumption levels compared to nutritional standards and peer group average intake levels and thus make people aware of the adequacy of their personal intake levels (Brug et al., 1999; De Bourdeaudhuij \& Brug. 2000). 


\section{For whom does computer tailoring work?}

Because most computer-tailored nutrition interventions to date have used (rather extensive) questionnaires for the personal survey and have provided respondents with written feedback, it has been argued that computer-tailored nutrition education may work only among more highly educated people and people who are already motivated to change their diet (Brug \& Van Assema. 2000). Indeed, health communication in general has been found to be more effective among more highly educated people (Preston, Baranowski, \& Higginbotham, 1988), and this may be even more true for written health communications. Non-motivated people (precontemplators) may not be willing to complete the survey or may not (seriously) read and process the tailored feedback, since they experience no 'need to change' and therefore no reason to participate in the intervention.

Most studies on the impact of computer-tallored nutrition education have indeed been conducted among self-selected samples and this typically results in over-representation of female, motivated and more highly educated respondents. indicating that, like health communications in general, computer-tailored nutrition education appeals more to highly educated, motivated women. However, one of our own studies was conducted in a workplace setting among a largely male employee population, and resulted in a $74 \%$ participation rate and significant reductions in fat intake in the tailored group (Brug et al., 1996), indicating that men can be interested in personalised feedback about their diet. Furthermore, a few larger studies have at least included sufficient numbers of men and less highly educated or non-motivated respondents. These studies provide the opportunity to test possible effect modifications of sex, stage of change and education. No sexintervention interactions have been reported for the impact of computer-tailored nutrition education. One study reported specifically on the impact of computertailored nutrition education among precontemplators and people with a relatively low educational level (Brug \& Van Assema, 2000). Computer tailoring proved to be more effective among precontemplators than general nutrition information. Furthermore, people in precontemplation made up $34 \%$ of the participants, indicating that many precontemplators do take an interest in personalised dietary feedback if and when it is offered to them. This finding suggests that, at least for dietary fat reduction, people in precontemplation may lack awareness rather than being uninterested/resistant to making changes. Computer tailoring was found to be as successful among people with lower education as it was among more highly educated people, and appreciation and exposure were even better among less highly educated respondents. Furthermore, the Health Works for Women and FoodSmart studies were conducted successfully among lower income and minority women (Campbell et al., 1999; Campbell, Tessaro, DeVellis, Benedict, Kelsey, et al., 2002). For poorly educated participants, literacy issues may interfere with the ability to use and understand tailored print material. However, for those with at least some reading ability, tailoring may help because there is less information to read and sift through, because information that is not personally relevant is eliminated. 
We have argued that the power of tailoring lies in its ability to make nutrition education more personally relevant. However, individualisation of nutrition education may not always be necessary to accomplish personal relevance. If the determinants of a specific dietary behaviour differ very little between people in a certain population, there should be no need to use computer tailoring. In such a population, well-designed generic materials that address these general determinants will be personally relevant for most of the target audience. Kreuter and colleagues found proof that when generic materials (by chance) fitted in well with the determinants and information needs of the respondents, their impact was as good as or better than that of tailored information (Kreuter, Oswald, Bull, \& Clark, 2000). This indicates that in populations or for dietary behaviours with only minor variations in behavioural determinants, there is no need to use individual tailoring techniques.

\section{The future of computer tailoring}

\section{Tailored print materials or interactive technology}

Most investigations into the impact of computer-tailored nutrition education to date have tested computer-tailored print materials (Brug \& Van Assema, 2000; Kreuter et al. 2000). Several limitations of printed tailored feedback, however. should be noted. Computer-tailored print materials only utilize part of the potential of computer tailoring. since interactivity and immediate feedback are not possible. Printed computer tailoring typically uses written surveys that are scanned into a computer, which then creates written feedback based on the individual survey results. The printed feedback, most often in the form of personal letters or individualised newsletters, is then sent by mail to the respondents. This procedure typically takes time and effort and requires at least a few days, but more often a couple of weeks, between completion of the survey and delivery of the feedback. The longer the period between survey and feedback, the greater the risk that the personal relevance of the feedback is diminished, since it may be possible that people change their beliefs. motivations or even their dietary behaviour in the time span between survey and feedback. Traditional printed computer-tallored feedback is also more expensive than generic nutrition education, since it requires at least some handling of the survey questionnaires and the feedback letters (Kreuter et al., 2000). On the other hand. it is possible that a strong point of printed feedback is its ability to be mailed or given directly to an individual from a trusted source. This may enhance its credibility and allows users to read it as many times as they wish and to share it with others.

A major limitation of computer tailoring is that it is difficult to mimic the positive characteristics of personal counselling beyond a certain point. Most computer-tailored interventions have not allowed for inclusion of direct interaction between the respondent and the nutrition education expert. Whereas computerized feedback 
has sometimes included tailored advice regarding social interaction with or social support from peers, this interaction is difficult to achieve without more intervention components directly aimed at involving social networks (Brug et al., 1999). As we have argued, the individualisation of messages may be one reason why computer-tailored nutrition education is effective. However, computer tailoring can also be criticised for its individual approach, since it lacks the social component that is present in interpersonal counselling (Brug et al., 1999). It has also been argued that personalised advice may not be effective since dietary habits are often not volitional or personally determined, since food is often bought or prepared by others. Attempts have therefore been made to conduct family-based tailored nutrition education, in which different family members received tailored feedback and were encouraged to discuss their feedback, especially with the person responsible for the cooking and the shopping. Although family-based tailored nutrition education has been found to be more effective then generic nutrition education (De Bourdeaudhuif \& Brug, 2000), a further study failed to prove the superiority of family-based tailoring over an individual tailored intervention (De Bourdeaudhuij et al., 2002).

Using interactive technology in computer tailoring may offer solutions to some of these issues: lower costs. better interaction, less time between screening and feedback, and opportunities for combining computer-tailored feedback with a socially supportive environment, e.g.. by offering opportunities to e-mail the 'expert' or participate in on-line discussion forums or chat sessions. Interactive technology allows participants to enter the answers to the survey questions directly into the interactive system by means of, for example, mouse clicks, keyboard, voice recording or touch screen video. Feedback is then given almost immediately on the (computer) screen (Oenema, Brug, \& Lechner, 2001). An early study using a webbased computer-tailored system showed that respondents who received interactive computer-tailored feedback appreciated their information more, were more aware of their fat intake levels and were more motivated to reduce their fat intake than respondents who received non-tailored information (Oenema et al., 2001). This study, however, did not attempt to compare the impact of the web-based feedback with tailored printed feedback.

The Internet and especially the World Wide Web are regarded as a very promising medium for health promotion interventions in general and may have great potential for the distribution of computer-tailored nutrition education in particular. In addition to individualised health promotion messages. Internet-based tailoring can incorporate access to vast information sources, communication with experts, peers, or early adopters of health promoting interventions, as well as links to providers and distributors of additional health promotion material (Street, Gold, \& Manning, 1997). On the other hand, we are faced with several challenges when using the Web for health promotion. The enormity of the Web, with its virtually unlimited amount of information. the limited possibilities to check the validity of the information. the sometimes doubtful sources of information. as well as the limitless opportunities to 'click' through to other web-sites on completely different topics, may all be barriers to bringing across credible and effective nutrition educa- 
tion messages (Oenema et al., 2001). Other potential pitfalls include people's tendency to 'surf the Internet rather than to spend concentrated time on one site, and the question whether people read differently and/or are less easily persuaded by electronic text than by traditional text on paper (Murphy et al., 2003). The real-life opportunities of Internet-based interactive tailored nutrition education therefore remain to be investigated.

\section{Future research}

The combination of greater effectiveness than general health education and the possibility of reaching larger numbers of people than interpersonal counselling makes computer tailoring a promising technique worthy of much further research. Several research gaps must be addressed to advance the field of computer tailoring. First, research is needed to test the impact of computer-tailored nutrition education by more objective outcome measures. To date, the available evidence is based on food consumption assessments with food frequency questionnaires and other self-reports. Studies are needed, and are currently being conducted, in which biomarkers, such as blood lipids and carotenoids, are used as effect indicators. Second, more research is needed to further reveal the processes responsible for the working mechanisms of tailored interventions, as it is not yet fully clear why tailored materials are more successful in influencing behaviour than non-tailored materials (De Vries \& Brug. 1999; Skinner et al. 1999). In this line of research. a number of so-called dismantling studies are underway or in the final planning phase. These studies compare the effects of tailored interventions that differ in the amount and/or content of the feedback provided. These studies should reveal which characteristics of tailored interventions increase its effectiveness, how far we should go with individualising feedback and when and where further differentiation no longer leads to a greater effectiveness or cost effectiveness. A study that recently started will test three tailored nutrition interventions. The first provides respondents with feedback about their intake levels of saturated fat. The second intervention gives additional normative information, that is, the respondents' intake levels are compared with recommendations as well as with the average intake levels of comparable others. The third intervention also provides respondents with suggestions on how to cut back on fat. This study is intended to show if personalised feedback about intake levels is sufficient to induce dietary change, or whether advice on how to make the required changes is necessary. In addition. studies are needed that test computer-tailored nutrition education against other state-of-theart intervention approaches, such as telephone counselling, social support interventions. and environmental interventions e.g., at point of purchase.

Further research is also needed to explore the opportunities for talloring to multiple health-related behaviours, based on different sources and/or communicated through different channels. Most studies on computer tailoring to date have investigated the potential of interventions aimed at changing one health-related behaviour. Computer-tailored interventions have been tested. for example. to encourage 
people to eat less fat, to stop smoking, to be more physically active, to participate in breast cancer screening, to encourage organ donor registration, or to seek protection from the sun (Brug \& De Vries, 1999: De Nooijer et al., 2002; Reubsaet, Brug. Kitslaar, Van Hooff, \& Van den Borne, in press; Skinner et al., 1999).

Some studies have targeted two or more health behaviours, such as consumption of fat, fruit and vegetables (Brug et al., 1998a; James et al., 2002). Attempts have been and are being made to develop broader computer-tailored interventions that address various life-style factors. There are several reasons for the development of life-style tailored interventions. Prevention of many contemporary health problems involves different life-style factors. Prevention of certain cancers may involve smoking cessation, dietary change as well as being alert to possible early cancer signs. Prevention of obesity and cardiovascular disease and probably also diabetes and certain cancers involve dietary as well as physical activity changes. The so-called WATCH project provided tailored information on multiple behaviours, f.e., diet, physical activity and colorectal screening. The tailored education resulted in improvements in all three cancer-preventive behaviours (James et al., 2002). A possible problem with tailored life-style interventions is that inclusion of more life-style factors necessitates more extensive surveys. People may be less willing to complete such surveys seriously or may be overwhelmed by too many recommendations for change. 'Phased' life-style tafloring systems are now being considered. in which participants first complete a brief screening instrument to detect the lifestyle factors that are most relevant to or of greatest interest to them personally. These factors are then surveyed more intensively, leading to the generation of computer-tailored feedback. Tailoring on the participants' choice of behavioural priority for change indeed appears to be a promising approach for tailored interventions (Campbell. Tessaro, DeVellis, Benedict, Kelsey, et al., 2000).

\section{Conclusion}

In conclusion, computer tailloring is currently one of the most promising and innovative approaches in nutrition education. Better exposure and more intensive cognitive processing as a result of individualisation and the self-evaluation properties of computer tailoring have been proposed as important causes of the effectiveness of computer-tailored nutrition education. However. little is known to date, and more research is needed, about when, why, where and for whom computer-tailored nutrition education is effective. 


\section{Web-based tailored nutrition education: Results of a randomised controlled trial}

\section{Abstract}

There is ample evidence that printed, computer-tallored nutrition education is a more effective tool for motivating people to change to healthier diets than generic nutrition education. New technology is now providing more advanced ways of delivering tailored messages, for example via the World Wide Web. Before disseminating a tailored intervention via the web, it is important to investigate the potential of web-based tailored nutrition education. The present study investigated the immediate impact of web-based computer-tailored nutrition education on personal awareness and intentions related to intake of fat, fruit and vegetables. A randomised controlled trial, with a pre-test-post-test control group design was conducted. Significant differences in awareness and intention to change were found between the intervention and control group at post-test. The taflored intervention was appreciated better, was rated as more personally relevant and had more subjective impact on opinion and intentions to change than the general nutrition information. Computer literacy had no effect on these ratings. The results indicate that interactive, web-based computer-tailored nutrition education can lead to changes in determinants of behaviour. Future research should be aimed at longer-term (behavioural) effects, and the practicability of distributing tailored interventions via the World Wide Web.

Published as: Oenema, A., Brug, J., \& Lechner, L. (2001). Web-based tailored nutrition education: results of a randomized controlled trial. Health Education Research, 16, 647-660. 


\section{Introduction}

Computer tailoring is a technique that has become quite popular in health education during the last decade (Kreuter et al., 2000). This is not surprising. since computer-tallored interventions were generally found to be more effective in changing relevant health-related behaviours than their non-tailored equivalents (Brug et al. 1999: Skinner et al., 1999: Strecher. 1999). Computer-tailored interventions mimic to some extent a classic tailoring technique: that of interpersonal counselling. In computer-tallored interventions, people are provided with information that is attuned to their personal characteristics, like health behaviours, motivations. attitudes and perceived barriers, thus making the information personally relevant. Since computer-tallored interventions document the diagnostic and educational expertise and techniques of the counsellor in a computer expert system, they lack the direct social support of interpersonal counselling. Computer tailoring. however, provides the opportunity to reach far more people, at far lower costs, than would be possible with interpersonal counselling. The combination of greater effectiveness than general health education and the possibility of reaching larger groups of people than interpersonal counselling makes computer tailoring an interesting method that is worth being explored both in greater depth and more extensively. Exploration in greater depth is required in order to further reveal the processes responsible for the working mechanisms of tailored interventions, as it is not yet fully clear why tailored materials are more successful in influencing behaviour than non-tailored materials (De Vries \& Brug. 1999; Kreuter et al., 1999; Skinner et al., 1999). More extensive exploration is needed in order to examine the possibilities for applying tailored messages to influence different health-related behaviours, using different information sources and communicating through different channels. The present study aims to contribute to the more extensive exploration. in that it explores the immediate impact of computer tailoring via a new channel.

The present paper reports the methods, results and implications of one of the first empirical evaluations of a so-called second-generation computer-tailored intervention: a nutrition education tool that is suitable for interactive use via the Worid Wide Web (WWW). Before presenting the study we will give a brief summary of why nutrition education is important and why computer-tailored. and therefore individualised, feedback may be especially effective for nutrition education.

\section{Diet and awareness in the Netherlands}

Diets low in saturated fat and high in fruit and vegetables are thought to reduce the risk of chronic diseases, like cardiovascular diseases and various cancers (Willet, 1994). Consumption of saturated fat is too high and intake of fruit and vegetables is far too low in the Netherlands (Voedingscentrum. 1998) as well as in most other 'Western' countries (Beer-Borst. Hercberg. Morabia. Bernstein, Galan, et al. 2000). In the Netherlands, fat consumption is responsible for approximately 37 percent of total daily energy intake, with $14 \%$ of total energy intake coming from 
saturated fat. These figures are substantially higher than the Dutch recommended values (30-35 \% energy from fat; $10 \%$ from saturated fat). The most recent Dutch national food consumption survey has shown that between 1988 and 1998, fruit and vegetable consumption decreased from 125 to 105 grams of fruit and from 141 to 123 grams of vegetables, while Dutch nutrition authorities recommend eating at least two pieces of fruit and 200 grams of vegetables per day (Voedingscentrum, 1998). Although the vast majority of the Dutch population eat too much fat and not enough fruit and vegetables, most people are not motivated to change to healthier diets (Brug et al., 1997). This lack of motivation to change has been attributed to the fact that most people are not aware of their unfavourable diets (Brug et al. 1997). A majority of Dutch adults are convinced that their diet is low in fat and provides sufficient quantities of fruit and vegetables while in fact, their intake of these food products is not in line with recommendations (Brug et al., 1994). This lack of awareness of personal (dietary) behaviour has also been found in other countries (Glanz et al., 1997) as well as for other complex health risk behaviours (Ronda, Van Assema, \& Brug, 2001) and has been identified as a major barrier in motivating people to change to healthier diets. Therefore, it is important to make people aware of their intake levels as a first step to behavioural change.

The importance of raising awareness is emphasised in Weinstein's Precaution Adoption Process Model (Weinstein, 1988). According to this model, awareness of risk behaviour emerges through three phases. If people are to become fully aware of a risk behaviour and are to become motivated to change this behaviour, they have to proceed through all three phases. Phase 1 is reached when people agree that they have heard of the (health) risk associated with a particular behaviour. for example that eating too much fat can cause cardiovascular diseases. Phase 2 is reached when people know that the risk behaviour is prevalent, i.e., when they are aware that many (other) people engage in this risk behaviour. Finally, phase 3 is reached when people acknowledge that they are engaging in the risk behaviour themselves (Weinstein. 1988). Where fat, fruit and vegetables are concerned, a majority of the Dutch population seems to have reached phase 2 , but not phase 3 . Most people in the Netherlands know that eating too much fat and too little fruit and vegetables, is bad for health, and they agree that many people eat too much fat and not enough fruit and vegetables. However, fewer than one-third of the population perceive their own diet to be too high in fat or too low in fruit and vegetables. According to Weinstein, the third stage of awareness can be achieved by giving personalised feedback on individual risk behaviour (personal feedback) as well as information about the risk behaviour of others (normative feedback) (Weinstein, 1988). As regards awareness of dietary habits, people should be provided with accurate information about their own fat, fruit and vegetable consumption levels, and those of others.

\section{Computer tailoring}

In tailored information, personal dietary habits can be compared with recom- 
mended intake levels (personal feedback). with the consumption patterns of peers (normative feedback) and with perceived risk behaviour. Furthermore, information can be provided which addresses beliefs and self-efficacy expectations with respect to healthier diets. Matching the information to personal characteristics can be achieved by linking up the results of a personal assessment or diagnosis with a message library, through a set of algorithms documented in a computer programme. The algorithms ensure that a set of messages tailored to the individual assessment is collected from the library. This set of messages forms the personally relevant information and can be presented in various ways, like for example a letter, a newsletter, or a magazine.

\section{The impact of computer-tailored interventions}

Several reviews of computer-tailored interventions have recently been published (Brug et al., 1999; Skinner et al., 1999: Strecher, 1999). Computer tailoring has proved successful in influencing smoking behaviours (Dijkstra, De Vries, \& Roijackers. 1998; Strecher, 1999), in encouraging participation in breast cancer screening (Rakowski et al., 1998: Skinner et al., 1994), and also in promoting healthy dietary habits (Brug et al., 1999).

The taflored nutrition interventions that have been described to date (Brug et al. 1999: Skinner et al., 1999) mainly aimed at reducing fat intake and increasing fruit and vegetable consumption. Results with regard to fat consumption have been quite consistent: tallored materials result in a significantly higher reduction of fat intake. Results for fruit and vegetable consumption, however, have been less straightforward. Some studies found a significantly higher increase in fruit and vegetable consumption in the tailored intervention groups (Brug et al., 1998a), whereas other studies did not find any intervention effects (Campbell, DeVellis, Strecher, Ammerman, DeVellis, \& Sandler, 1994: Lutz, Ammerman. Atwood. Campbeili, DeVellis, \& Rosamond, 1999). Most studies found that tailored materials were remembered, appreciated and read better than non-tailored materials (Brug et al., 1998a: Campbell et al., 1994). These results show that tailored nutrition interventions can have a positive influence on dietary change.

\section{First- versus second-generation tailored interventions}

Most computer-tailored interventions that have been used to date have provided people with printed materials. This is referred to as first-generation tailoring (Skinner et al., 1999: Strecher, 1999). As technology proceeds, a second-generation of more advanced ways of delivering tallored messages is feasible. The World Wide Web has been proposed as a very promising channel for delivering computer-tailored feedback. However, before disseminating interactive computer-tailored interventions via the web, the effectiveness of this method should first be established. The present study aimed at exploring the immediate impact of a web-based computer-tailored nutrition education programme for adults, on self-rated intake levels of 
fat. fruit and vegetables and on motivation to change these dietary behaviours, as compared with a non-tailored intervention.

We hypothesized that:

- The web-based tailored intervention would have a greater impact on self-rated intake and motivation to change than the non-tailored intervention:

- The web-based tailored intervention would be appreciated better than the nontailored intervention;

- Appreciation of the intervention would be higher among respondents who were more experienced in using the computer.

\section{Methods}

\section{Participants}

Participants were recruited from among the employees and students of various institutes for (adult) education. Potential participants received a letter prior to the study, explaining its purpose and general procedure. After this letter, the employees were sent an e-mail, to which they could reply to indicate their willingness to participate in the study. The students were asked during one of their classes if they were willing to participate in the study. The only exclusion criterion was insufficient understanding of Dutch. During recruitment, we made it particularly clear that people with no explicit interest in nutrition should also enrol.

Participants were offered a $€ 9$ gift voucher and could win a trip to Paris for two that was to be raffled among all the participants of the study.

\section{Study design and procedure}

A pre-test-post-test randomised controlled trial was conducted to compare the tailored intervention with a control condition. Appointments were made with the people that had indicated their willingness to participate in the study. Respondents were exposed to the interventions on site, either in classrooms or offices. In both cases, care was taken that respondents could not see or communicate with each other. After the participants had received information about the study and had given their informed consent, the procedure of the study was explained in further detail. The participants were randomly assigned to one of the conditions by drawing lots. Respondents in the intervention group then started going through the web-based tailored programme. i.e., filling out the assessment and reading the feedback. Respondents who were not experienced in using computers were given a brief instruction on how to proceed through the programme. After respondents had finished the programme, a written post-test questionnaire was handed out. Respondents in the control group were first asked to fill out a written baseline questionnaire. Subsequently a written general nutrition information letter was 
handed out to them to read, after which they were asked to fill out a post-test questionnaire. In the present study participants could not take a print out of the tailored information or a copy of the letter home with them.

\section{Measurement}

The baseline questionnaire was an 84-item self-administered questionnaire, consisting of three parts. The first part assessed information about age, sex, weight, height, educational level, living situation (alone/together), use of special diets and whether or not the respondent regularly did the cooking or the shopping for the household. The second part was a validated food frequency questionnaire, which assessed the frequency of use of 19 products or product categories (i.e., dairy products, bread spreads, cheese, meat, gravy, sweet snacks and savoury snacks) that contribute most to saturated fat intake, as well as the quantities and types of product (high fat, medium fat, or low fat). The answers to the food frequency questionnaire were used to calculate a score for fat, ranging from 0 to 80 . This score is a reflection of total saturated fat intake. One point resembles about two grams of saturated fat and thus a high score reflects a high saturated fat intake. The advantage of this screening instrument is that it is short and easy to administer, while it still enables the ranking of respondents according to individual fat intake and the detection of changes in individual fat consumption (Van Assema, Brug. Kok. \& Brants, 1992). The frequency and quantity of fruit and vegetable consumption were assessed by a total of ten questions on the frequency (how many days a week) and quantities of vegetables (cooked and raw) and fruit (citrus fruit, other fruit and fruit juice) usually consumed. This information was used to calculate the average number of servings of vegetables and fruit per day. The contents and validity of this food frequency questionnaire have been described in more detail elsewhere (Van Assema et al., 1992; Van Assema, Brug, Ronda, \& Steenhuis, 2001).

The third part of the questionnaire assessed the respondents' self-rated intake levels of fat, fruit and vegetables, which were used as a reflection of awareness of personal intake levels. Self-rated intake was assessed at two levels: self-rated intake and self-rated intake compared to others. Both were measured by means of one question (see Table 3.1). Stage of change, attitudes and self-efficacy related to reducing fat intake and increasing fruit and vegetable consumption were also assessed at baseline, as these factors were used to tailor the feedback (see Table 3.1),

The post-test questionnaire consisted of 49 items (intervention) or 46 items (control). The latter questionnaire was slightly shorter because some specific questions on the attractiveness and usability of the nutrition intervention could not be used in the control group. The post-test questionnaire consisted of two parts. The first part included the same self-rating and staging items as the baseline questionnaire, with extra items to assess intention to change fat, fruit and vegetable intake on seven-point scales (see Tables 3.1 and 3.2). The second part of the post-test questionnaire consisted of questions on the appreciation, relevance, credibility and 
Table 3.1. Overview of psychosocial questions

Self-rated intake

- How much fatfruit/vegetables do you think you eat? (self-rated intake)

- Do you think that the amounts of fat/fruit/vegetables you eat are larger or smaller than those of other people of your age and sex? (self-rated intake compared to others)

General attitude

- Do you think it is good or bad to eat less fat/more fruit/more vegetables?

Self-efficacy ${ }^{2}$

- Do you think it would be easy or difficult for you to eat less fat/more fruit/more vegetables?

Stages of change ${ }^{b}$

- Do you intend to start eating less fat/more fruit/more vegetables within half a year from now?

- Do you intend to start eating less fat/more fruit/more vegetables within a month?

- Do you already eat less fat/more fruit/more vegetables?

- Did you try to eat less fat/more fruit/more vegetables before?

- Did you continue eating less fat/more fruit/more vegetables since you started to do so?

Note. ${ }^{a}$ Measured on five point scales ranging from very little/much smaller - very much/much larger,

${ }^{b}$ Measured in a yes/no format.

use of the intervention (see Table 3.3). These questions had to be answered on 100$\mathrm{mm}$ visual analogue scales with 'completely agree' and 'completely disagree' as the two extremes. Finally. there were questions about the frequency and ease of computer use, and familiarity with the Internet (how often do you use a computer/the Internet? How easy is it for you to use a computer/find something on the Internet?). to be answered on six- and five-point scales respectively.

\section{The tailored intervention}

Generating a computer-tailored nutrition message requires three inter-related components: a theory-driven diagnostic tool to assess feedback goals for each participant, a message library containing feedback messages for all possible diagnoses and a computer programme that selects the feedback messages that correspond with each specific diagnosis (Brug et al., 1999). These same elements are necessary for a web-based tailored intervention, but a web-based intervention offers more flexibility as well as more opportunities for interaction. For example, it is not necessary to complete the whole diagnosis before feedback is given; not only the feedback, but also the diagnostic tool can be taflored by skipping questions that are not relevant to a respondent; the amount and specificity of the feedback can be tailored to the respondent's need for information at a specific time, by giving the respondent a choice to answer more questions and receive more specific information on a particular topic.

The diagnostic tool used for the present intervention was the baseline questionnaire described in the previous subsection. The questionnaire was divided into three separate parts, one for fat, one for fruit and one for vegetables, thus creating. three short questionnaires. 
The programme started with a home page describing what a tailored nutrition education programme is, for whom this particular programme was made, who produced it and how it should be used. After reading this home page, a respondent could start the programme by clicking on a button in the menu bar on the left side of the screen (see Figure 3.1). For the purpose of the present study, we asked respondents to follow the sequence in the menu bar, which starts with fat, followed by vegetables, fruit and recipe suggestions, although a random sequence is possible.

Each topic started with a short introduction on the subject, for example why low fat diets are important and that it is important for everybody to have diets low in fat. This introduction was followed by the diagnosis section. Questions appeared on the screen one by one, with the next question appearing as soon as the previous one had been answered. Questions that were irrelevant to a particular person were skipped. For example, vegetarians were not asked questions on meat consumption. Thus the questionnaire could differ in length, depending on the individual diet. After all relevant questions had been answered, the feedback appeared on the screen. in an attractive layout, with a coloured background and illustrations accompanying the texts. Respondents could proceed to the next topic by clicking the next button in the menu bar.

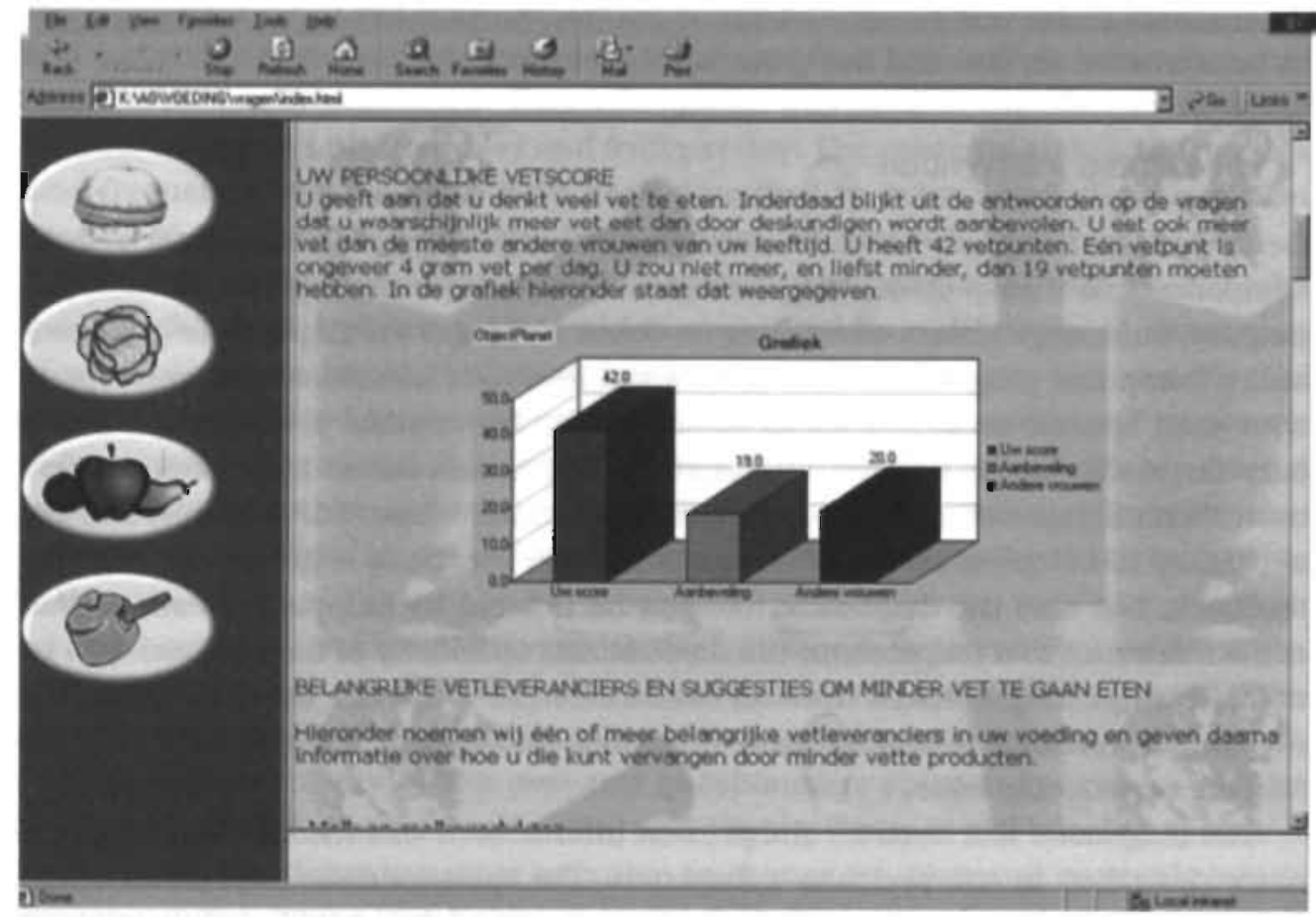

Figure 3.1. Part of the feedback section on fat. 
Each feedback section started with a short introduction and an overview of topics to be discussed. Feedback messages were given on the respondent's own estimation of fat intake and the fat score computed from the baseline questionnaire. The respondent's personal fat score was compared with recommended intake levels, and respondents who underestimated their fat intake were confronted with their misconception. If the fat score exceeded the average fat score of others of the same age and sex, the personal fat score was also compared with that of others. This information was visualised in a graph (see Figure 3.1). Subsequently, messages were provided on the most important sources of fat in the diet and low fat alternatives. Messages were available on the major sources of fat in the Dutch diet (milk products, bread spreads, cheese, meat. gravy and sauce, savoury snacks and sweet snacks), but respondents only received information on fat sources that featured prominently in their own diet.

The information on fat ended with a message tailored to stages of change, motivating people in precontemplation to reconsider their fat intake, encouraging respondents in contemplation and preparation to proceed to action, and reinforcing respondents in action and maintenance to continue with their changes. Messages differed for positive, negative or neutral attitudes and positive, negative or neutral self-efficacy expectations. Respondents who thought it would be difficult for them to eat low fat food in five particular situations (when in a restaurant, when invited by family or friends, when at a party, when hungry between meals), were given messages on how to face these difficult situations.

Feedback on vegetable intake was given by comparing the computed vegetable intake with the recommended quantity of 200 grams of vegetables each day. Respondents who did not meet the recommended levels were given suggestions on how to increase consumption by either eating vegetables more frequently or in larger portions. Suggestions were made to eat vegetables with other meals, as well as tips for easy ways to increase vegetable consumption. For those who did not meet the recommendation for vegetables, portion sizes for three different kinds of vegetables were given, both in words and photographs. The information on vegetable consumption also ended with a message tailored to stage of change.

Computed fruit consumption levels were compared with recommended levels and self-rated intake levels. The messages emphasised the importance of eating at least two pieces of fruit each day. Respondents who did not meet the recommendations were given suggestions on how to increase fruit intake, tallored to the time of day fruit was usually eaten. Again, the information ended with a message tailored to stages of change.

Finally, the recipe part offered 56 recipes for low fat and high vegetable main courses, desserts and snacks, which respondents could browse through. This part could be entered either by clicking the button in the menu bar or via links in the information sections on fat, fruit and vegetables. The message library has been tested in earlier studies (Brug et al., 1998a; Brug \& Van Assema, 2000) and was revised and extended for the present study.

A computer programme, written in Visual Basic, enabled the creation and stor- 
age of all essential parts of a tailored intervention (screening instrument, message library, tafloring algorithms, and layout of the final feedback). Java script routines generated the tailored messages and created a website consisting of HTML pages. The programme was created for use on the Internet, but for the purpose of the present study we had the programme installed locally on hard disk, to make sure people would use the programme and not surf to other sites. Internet explorer 5.0 was the browser used to run the programme. The web-based programme was pre-tested in two sessions and changes were made according to the findings of the pre-tests.

\section{Control condition}

Respondents in the control condition received a non-tailored nutrition information letter, with information derived from brochures of the Netherlands Nutrition Centre. The letter provided information on the importance of a healthy diet and on the fact that Dutch people eat too much fat and not enough fruit and vegetables, but that many people do not know they have unfavourable diets themselves. The section on fat listed the risks of high fat diets and emphasised that everybody who eats too much fat is at risk. Low fat alternatives were given for some high fat products that are very common in the Dutch diet. The fruit and vegetable part emphasised the positive consequences of eating more fruit and vegetables, followed by suggestions for increasing fruit and vegetable intake. The letter ended with two low fat recipes. The four-page letter was illustrated with cartoons.

\section{Statistical analysis}

Equality of study groups at baseline was tested by means of logistic regression analyses with the study group as the dependent variable and age, gender, consumption of fat, fruit and vegetables, self-rated intake and self-rated intake compared to others as the independent variables.

Analyses of variance were used to test for differences in intention to change to low fat and high fruit and vegetable diets between study groups at post-test. To test for differences between study groups in post-test self-rated intake levels analyses of co-variance were conducted, adjusting for the baseline self-rated intake levels. Since people with unfavourable diets should profit in particular from nutrition interventions, the same analyses were conducted separately for subgroups that did not meet recommended intake levels at baseline. Chi-square tests were used to test for differences in proportions of respondents who did not meet recommended intake levels in both study groups.

Differences in use, subjective estimation of impact and appreciation of the tailored nutrition information programme and the general nutrition information letter were tested using $t$-tests.

To detect differences in the appreciation of the tailored nutrition information programme between computer-literate and computer-illiterate respondents. Pear- 
son correlations were computed between computer literacy (calculated as the sum of frequency and ease of computer use, Pearson $r=.80$ ) and the attractiveness as well as ease of use of the programme.

Differences with $p<.05$ were considered to be significant. Analyses for intervention effects were one-tailed, since the superiority of the tailored intervention was hypothesized. The analyses were only conducted when no substantial violations of normal distribution were found.

\section{Results}

\section{Participants}

A total of 204 respondents participated in the study. Due to technical problems. the data of four subjects in the tailored intervention group were lost. Information on the fat consumption of two other respondents in the tailored intervention group was not recorded, although the subjects completed the whole programme. Two respondents only completed the section on fat. Of these four respondents the data on the completed and recorded topics were included in the analyses. Thus, most analyses were conducted on 96 subjects in the tailored intervention group and 102 subjects in the control group.

\section{Baseline measurements}

Approximately two-thirds of the respondents were female $(62 \%)$. Mean age was 44 years $(S D=9.7)$. Almost half of the respondents had a college or university degree (47\%), while $26 \%$ had completed medium level or lower professional education, $22 \%$ had graduated from high school and $5 \%$ had no education beyond primary school.

The average fat score was $18.5(S D=6.3)$. while the average vegetable intake and fruit consumption was 198.5 grams (2.5 servings, $S D=103.2$ grams $)$ and 2.2 pieces (2.2 servings, $S D=1.7$ pieces) per day respectively. Logistic regression analyses found no differences between the study groups in these average intake levels or in other key variables at baseline.

Fifty-two (54\%) respondents in the tailored intervention group and $44(43 \%)$ in the control group exceeded the recommended levels for fat intake, while 47 (49\%) and $45(47 \%)$ respondents in the tailored intervention group and $53(52 \%)$ and $53(52 \%)$ respondents in the control group did not meet the recommended intake levels for vegetables and fruit respectively. The percentage of respondents in each subgroup did not differ significantly between the study groups.

\section{Impact on self-rated intake and motivation to change}

Analyses of (co)variance showed significant differences in the intention to eat less 
fat and in self-rated fat intake compared to others (Table 3.2). Respondents in the tailored intervention group had a significantly greater intention to eat less fat [F[1. $196)=5.85, p=.008$ ] and were significantly less likely to think that they ate less fat than others $[F(1,195)=5.52, p=.010]$. Self-rated fruit intake $[F(1,194)=7.63, p=$ $.003]$ and self-rated fruit intake compared to others $[F[1,193]=2.97, p=.044]$ were significantly higher in the tailored intervention group. indicating that respondents in the tallored intervention group were significantly less negative about their fruit consumption.

\section{Use, subjective impact and appreciation}

Respondents in both groups read most of the nutrition information and both groups rated the information equally attractive to read (Table 3.3). The tailored nutrition information programme, however, was more likely to be used again [t(192) $=2.47, p=.008$ !

Respondents in the tailored intervention group were more likely to have changed their opinion about their diet $[t(193)=3.82, p=.000]$ and more likely to express the intention to change their diet $[t(195)=3.35, p=.001]$ as a result of the nutrition information.

Although both groups were equally positive in their answers to the questions whether the nutrition information was clear, credible and interesting, respondents in the taflored intervention groups rated the information about all three topics as more personally relevant $[t(180)=3.52, p=.001$ for fat: $t(184)=3.27, p=.001$ for vegetables: $t(187)=1.91, p=.029$ for fruit]. more specifically intended for them $[t(190)=6.92, p=.000$ for fat: $t(191)=6.76, p=.000$ for vegetables: $t(189)=4.91$, $p=.000$ for fruit], and newer to them $[t(172)=4.61, p=.000$ for fat: $t(171)=4.05$, $p=.000$ for vegetables; $t(172)=3.81, p=.000$ for fruit $\mid$ than did respondents in the control group.

Computer literacy did not correlate significantly with the attractiveness of the web-based tallored intervention (Pearson $r=.02$ ). indicating that the programme was rated equally attractive by both computer-literate and computer-illiterate respondents, but a significant negative correlation was found between computer literacy and ease of use of the programme (Pearson $r=-.27, p<.01$ ), indicating that the programme was more difficult to use for computer-illiterate respondents.

\section{Discussion}

The web-based tailored nutrition information programme we tested in this study resulted in some significant differences between the tailored intervention group and the control group as regards the self-rated intake levels of fat, fruit and vegetables, and in the intention to change to healthier diets. Effects occurred both in the total study population and in the sub-groups of respondents with unfavourable diets. Furthermore, the tailored intervention was evaluated more positively 
Table 3.2. Mean values (and SD) for key variables at baseline and post-test for the total study population and for respondents with intake levels of fat, fruit and vegetables not in accordance with recommendations

\begin{tabular}{|c|c|c|c|c|c|c|c|c|}
\hline \multirow[b]{3}{*}{ Variable: } & \multicolumn{4}{|c|}{ Total study population } & \multicolumn{4}{|c|}{$\begin{array}{l}\text { Respondents with intake levels not in accordance with } \\
\text { recommendations }\end{array}$} \\
\hline & \multicolumn{2}{|c|}{ Baseline } & \multicolumn{2}{|c|}{ Post-test } & \multicolumn{2}{|c|}{ Baseline } & \multicolumn{2}{|c|}{ Post-test } \\
\hline & $\begin{array}{l}\text { Tailored } \\
(n-96)\end{array}$ & $\begin{array}{l}\text { Control } \\
(n-102)\end{array}$ & $\begin{array}{l}\text { Tailored } \\
(n-96)\end{array}$ & $\begin{array}{c}\text { Control } \\
(n-102)\end{array}$ & Tailored" & Controle & Tailored & Control" \\
\hline Self-rated fat intakes & $0.03(0.73)$ & $-0.23(0.77)$ & $0.17(0.68)$ & $0.01(0.81)$ & $0.02(0.73)$ & $0.07(0.62)$ & $0.38(0.66)$ & $0.32(0.67)$ \\
\hline $\begin{array}{l}\text { Self-tated fat intake compared to } \\
\text { others }\end{array}$ & $-0.31(0.70)$ & $-0.44(0.77)$ & $-0.05(0.80)$ & $-0.33(0.74)^{b}$ & $-0.35(0.65)$ & $-0.16(0.68)$ & $0.19(0.82)$ & $-0.09(0.65)^{b}$ \\
\hline Intention to eat less fat ${ }^{d}$ & - & - & $0.72(1.21)$ & $0.29(1.26)^{b}$ & - & - & $0.96(1.15)$ & $0.61(1.20)$ \\
\hline Self-rated vegetable intakes & $0.20(0.71)$ & $0.37(0.73)$ & $0.08(0.74)$ & $0.30(0.76)$ & $-0.06(0.70)$ & $0.11(0.64)$ & $-0.19(0.77)$ & $0.04(0.73)$ \\
\hline $\begin{array}{l}\text { Self-rated vegetable intake com- } \\
\text { pared to others? }\end{array}$ & $0.18(0.74)$ & $0.30(0.78)$ & $0.08(0.82)$ & $0.27(0.72)$ & $0.00(0.81)$ & $0.04(0.76)$ & $-0.21(0.86)$ & $0.06(0.63)^{4}$ \\
\hline Intention to eat more vegetables ${ }^{d}$ & - & - & $0.22(1.33)$ & $0.10(1.21)$ & - & - & $0.64(1.15)$ & $0.15(1.23)^{5}$ \\
\hline Self-rated fruit intakes & $-0.49(0.91)$ & $-0.51(0.98)$ & $-0.27(0.93)$ & $-0.49(0.97)^{b}$ & $-0.91(0.85)$ & $-1.00(0.73)$ & $-0.78(0.90)$ & $-1.00(0.73)$ \\
\hline $\begin{array}{l}\text { Self-rated fruit intake compared to } \\
\text { others }\end{array}$ & $-0.31(0.93)$ & $-0.34(1.01)$ & $-0.16(0.89)$ & $-0.34(0.96)^{4}$ & $-0.67(0.88)$ & $-0.87(0.84)$ & $-0.64(0.77)$ & $-0.81(0.84)$ \\
\hline Intention to eat more fruit ${ }^{d}$ & - & - & $0.58(1.32)$ & $0.51(1.34)$ & - & - & $1.27(1.01)$ & $0.75(1.27)^{4}$ \\
\hline
\end{tabular}

Note. 4 Significant difference between tailored intervention and control group at post-test $p<.05$, bignificant difference between tailored intervention and control group at post-test $p<.01$, "Measured on a scale ranging from -2 to +2 (very low/much lower - very high/much higher), "Measured on a scale ranging from -3 to +3 idefinitely not - definitely). "Numbers of respondents in each sub-group are mentioned in the baseline measurement section, 'Respondents who do not meet the relevant recommendation. 
Table 3.3. Use, subjective impact and appreciation of the tailored and general nutrition information (means and standard deviation)

\begin{tabular}{|c|c|c|c|c|c|c|}
\hline & $\begin{array}{l}\text { Tailored } \\
(n-96)\end{array}$ & $\begin{array}{c}\text { Control } \\
(n-101)\end{array}$ & & & & \\
\hline I read all of the nutrition information & $7.0(11.6)$ & $5.1(11.9)$ & & & & \\
\hline $\begin{array}{l}\text { I would consult the programme/letter again if I had the } \\
\text { opportunity }\end{array}$ & $29.7(32.5)$ & $41.9(36.4)^{b}$ & & & & \\
\hline The nutrition information was attractive to read & $15.0(16.7)$ & $19.5(22.1)$ & & & & \\
\hline $\begin{array}{l}\text { I changed my opinion about my diet as a result of the nutrition } \\
\text { information }\end{array}$ & $40.2(28.5)$ & $56.9(32.9)^{\mathrm{k}}$ & & & & \\
\hline \multirow{3}{*}{$\begin{array}{l}\text { I intend to change my diet as a result of the nutrition } \\
\text { information }\end{array}$} & $35.2(28.6)$ & $49.6(31.8)^{6}$ & & & & \\
\hline & \multicolumn{2}{|c|}{ For fat } & \multicolumn{2}{|c|}{ For vegetables } & \multicolumn{2}{|c|}{ For fruit } \\
\hline & $\begin{array}{l}\text { Tailored } \\
(n=95)\end{array}$ & $\begin{array}{l}\text { Control } \\
(n-101)\end{array}$ & $\begin{array}{l}\text { Tailored } \\
(n-95)\end{array}$ & $\begin{array}{l}\text { Control } \\
(n-101)\end{array}$ & $\begin{array}{l}\text { Tailored } \\
(n=94)\end{array}$ & $\begin{array}{c}\text { Control } \\
(n-101)\end{array}$ \\
\hline The information was clear & $10.8(16.4)$ & $7.7(12.0)$ & $9.3(11.9)$ & $8.7(13.3)$ & $8.3(11.0)$ & $8.1(13.2)$ \\
\hline The information was of personal relevance to me & $24.9 \cdot(23.7)$ & $39.4(33.7)^{c}$ & $29.5(26.6)$ & $44.1(35.7)^{c}$ & $28.2(26.5)$ & $36,6(34.4)^{\circ}$ \\
\hline The information was specifically intended for me & $25.9(26.2)$ & $54.8(32.3)^{c}$ & $30.5(27.9)$ & $60.2(33.4)^{\mathrm{k}}$ & $29.6(27.5)$ & $51.3(34.1)^{c}$ \\
\hline The information was credible & $14.9(21.4)$ & $12.0(18.8)$ & $12.4(14.6)$ & $9.7(15.6)$ & $12.2(16.4)$ & $10.9(16.2)$ \\
\hline The information was new to me & $61.9(34.3)$ & $81.8(25.2)^{\mathrm{K}}$ & $66.8(32.0)$ & $83.1(23.2)^{6}$ & $68.5(30.5)$ & $83.3(22.9)^{c}$ \\
\hline The information was interesting & $21.8(22.2)$ & $25.5(25.3)$ & $26.7(26.5)$ & $24.7(25.5)$ & $24.5(25.1)$ & $23.0(24.1)$ \\
\hline
\end{tabular}

Note, Scores on visual analogue scales ranging from 0 (completely agree) to 100 (completely disagree). " Significant difference $p<.05$, b Significant difference $p<$ .01 , 'significant difference $p<.001$. 
on important criteria for effective dietary interventions (Contento et al., 1995): personal relevance, matched to the individual, and novelty of information. The tailored intervention was attractive to computer-literate as well as computer-illiterate respondents.

The present study is the first to assess the impact of web-based computer-tailored feedback and one of very few studies to assess the immediate impact of the intervention. Since almost all studies on computer-tallored nutrition education have assessed short-term ( 1 month) or longer term ( $>4$ months) changes as well as primarily behavioural changes (Brug et al., 1999), the present study is not readily comparable to these earlier studies. Nevertheless, similar to other studies on the impact of computer talloring, tailoring effects on intentions to change to healthier diets were found. The focus of the present study was specifically on increasing awareness of personal dietary intake, and tailoring effects were found on self-rated intake levels, which are a reflection of awareness. Since awareness of personal intake has been identified as a prerequisite for proceeding to dietary change, these results are certainly relevant and are in line with findings from a recent study by De Bourdeaudhuij \& Brug (2000).

From earlier studies we have learned that tailored nutrition interventions are generally more effective in inducing dietary change than non-tailored interventions. The present study provides some evidence that a second-generation, interactive. computer-tailored intervention also influences factors that are important determinants of dietary change. The web-based tailored intervention that was tested had, at least, a greater immediate impact and was better appreciated on important features than general nutrition information. Although this information on the immediate impact is promising, results are limited to effects on pre-behavioural determinants and we lack information on longer-term and behavioural effects. Our future research will aim at studying the longer-term effects of the intervention on awareness, intention and behaviour.

There are some further limitations to this study that should be taken into consideration in interpreting the results. The impact of the intervention was studied in a controlled situation. The programme was locally run and there were no possibilities of linking to other pages or sites outside the programme. Furthermore, we instructed the respondents to follow the programme in a predetermined sequence and to complete the whole programme. This gave us the opportunity to study the results of the programme, but in real life settings the programme may be used differently, there may be distraction from the programme to other sites and there may be other confounding factors which all could lead to different results. Generalisability of the results of this study to other populations is limited, because our sample was not representative of the entire Dutch population. Although we approached potential respondents by direct mail or through face-to-face contact. asking them to participate in our study, the sample was nevertheless self-selected. Even though we emphasised that anybody could participate in the study including people with no particular interest in food and nutrition, we may have selected a sample of respondents who were more than usually interested in nutrition and diet. 
Indeed the proportion of respondents with unfavourable diets in the present study was somewhat lower than that in the general Dutch population (Voedingscentrum, 1998), although we still attracted many people who did not meet the dietary recommendations. Further, we used an electronic questionnaire as part of the tailored intervention, and a written questionnaire in the control group. Studies investigating the comparability of web-based questionnaires to paper and pencil questionnaires. however, have found no differences between the two (Davis, 1999). but we cannot be certain whether this was also true in our study.

To our knowledge, no other studies on the impact of web-based computer tailored interventions have been published to date. The level of impact in the present study is therefore hard to judge. Some studies have reported on the impact and appreciation of non-tailored Internet-based programmes. These studies showed promising results and the authors were positive about the Internet as a channel to deliver health education messages (e.g., Celio, Winzelberg. Wilfley, Eppstein-Herald, Springer, et al. 2000; Winzelberg, Eppstein, Eldredge, Wilfley, Dasmahapatra, et al., 2000).

The advantages of a web-based, interactive, computer-tailored intervention over a more traditional print version are multiple. First of all, immediate feedback can be given, which makes it more likely that the information is indeed matched to the respondent's level of awareness, dietary pattern, beliefs and motivations at that particular time. In the more traditional settings, where printed materials were used, an interval of two weeks or more between assessment and feedback is not unusual. As beliefs and motivations may very well change in such a period of time, the information may no longer be tailored at the time respondents read their feedback. Furthermore, because of its more interactive properties, second-generation tailored feedback is even more similar to interpersonal counselling, which may enhance the effect of the intervention. Another advantage is that, once on the web, the failoring programme can reach a large group of people with a minimum investment in human effort and may therefore be cost-effective. A web-based computertailored intervention is flexible and can be updated continuously, thus making it possible to tailor to the most recent knowledge of, for example, determinants of dietary change or new products on the food market. As regards research applications. web-based tailored interventions can be very useful in experimental studies of the working mechanism of the tailoring process. Because of its flexibility it is relatively easy, for example. to change the assessment or change the messages, thus creating different conditions.

A disadvantage is that an intervention provided via the World Wide Web reaches only people who have access to the Internet. Furthermore information read from a screen may not be as thoroughly processed as information read as printed material.

The present study aimed to investigate the potential immediate impact of a web-based tailored nutrition intervention. as it is important to assess its effectiveness before further dissemination. We did not study how the programme could be used on the web. how to reach the target population via the web and how to 
ensure perceived credibility of the information when provided via the web. It is important, however, to investigate these issues in future research, since merely making a promising programme available via the Internet will probably not result in the use of the programme by the target audience. For example, it may not be easy to attract people to the web-site. This applies especially to people who are not aware of their unfavourable diets. They may initially not be interested in web-sites promoting dietary change, although studies on first generation computer-tailored feedback do indicate that even people who think their diets are in accordance with recommendations are interested in personal feedback and do volunteer to receive tailored messages (Brug \& Van Assema, 2000). Furthermore, when people do visit the website they should be encouraged to stay there long enough to complete the screening questions and to read the feedback and people should be convinced that the web-site is produced by a reliable source.

In spite of the limitations of the present study and the issues discussed, it can be concluded that a web-based tailored intervention can have an impact on determinants of dietary change and that the present findings warrant further studies on the impact and advantages of web-based tailored interventions in promoting healthy nutrition. 


\title{
Feedback strategies to raise awareness of personal dietary intake: Results of a randomised controlled trial
}

\begin{abstract}
Background - Feedback is a strategy that can be used to influence awareness of dietary habits. Feedback was applied in an interactive computer-tailored intervention and in printed self-test forms. Methods - A randomised controlled trial with a 3 (study groups) by 2 (higher vs. medium or lower educational level) design was conducted. Adult subjects $(N=304)$ were randomly assigned to a feedback group or the control group. Immediate impact on realism of self-rated intake levels of fat. fruit and vegetables were tested, as were intentions to change. Results - Self-rated fat intake compared to others was more realistic among respondents with a medium or lower educational level in the tailored intervention group. Self-rated fruit intake compared to others was more realistic in the tallored intervention group. Self-rated fat intake was more realistic in the tailored intervention than the self-test group. Intention to reduce fat consumption was greater in the tailored intervention group. Intention to eat more vegetables was greater in the tailored than in the self-test group. Subjects rated the tailored intervention as more effective, more personally relevant, more individualised and providing more new information. Conclusions Only the tailored intervention had an immediate impact on awareness and dietary change intention and was appreciated better than both other interventions.
\end{abstract}

Published as: Oenema A., \& Brug, J. (2003). Feedback strategies to raise awareness of personal dietary intake: results of a randomized controlled trial. Preventive Medicine, 36, 429-439. 


\section{Introduction}

Intake levels of (saturated) fat are above, while intake levels of fruit and vegetables are below recommendations in the Netherlands, as well as in most other Western countries (Beer-Borst et al., 2000; Ganji \& Betts, 1995). Lack of awareness of personal dietary habits has been identified as a major barrier in motivating people to change to healthier diets (Brug et al., 1994: Lechner et al., 1997). To overcome this barrier, it is essential to find and test strategies that increase awareness. Weinstein's Precaution Adoption Process Model (Weinstein, 1988) emphasises the importance of awareness in behavioural change, and proposes personalised and normative feedback to influence awareness. The present study applied personalised and normative feedback in two interventions: 1) an interactive computer-tailored programme providing extensive feedback based on a thorough diagnosis and 2) printed self-test forms, providing brief feedback based on a short and easy to administer self-diagnosis. The effects of both interventions were compared with those in a control group that received general nutrition information, in a randomised controlled trial. Before presenting and discussing the results, we introduce the rationale and theoretical foundation of the study.

Diets low in (saturated) fat and high in fruit and vegetables are associated with a decreased risk of cardiovascular disease and cancer (Willet. 1994). The Health Council of the Netherlands recommends diets with no more than 10 percent of energy intake from saturated fat and no more than 35 percent of energy intake from total fat. The latter officially only applies to people with or at risk for becoming overweight. This is, however, the majority of the population. Recommendations for vegetables and fruit are at least 200 grams (2.5 servings) and two pieces ( 2 servings) each day (Health Council of the Netherlands, 2001). According to the most recent national food consumption survey, intake levels of fat are substantially higher. while intake leveis of fruit and vegetables are substantially lower than these recommendations. Average intake of saturated fat constituted 14 percent of the energy intake, and that of total fat 37 percent. Average intake levels of vegetables and fruit were 123 (1.5 servings) and 105 grams (1 serving) respectively (Voedingscentrum, 1998). Contrary to these 'objective' findings, many people are convinced that their diets are healthy and therefore do not experience a need to change (Brug et al.. 1997; Glanz et al., 1997). The discrepancy between more objectively measured intake levels and self-rated intake levels can be defined as lack of awareness, which is a barrier for motivation to change.

The role of awareness is emphasised in Weinstein's Precaution Adoption Process Model as a prerequisite of contemplating behavioural change (Weinstein, 1988). The model describes three phases through which awareness of personal risk behaviour emerges. For a person to become fully aware (phase three) of a personal risk behaviour (e.g., my personal intake of saturated fat is too high), it is important that they have first heard of the health risk associated with that behaviour (phase one; e.g., high saturated fat intake can increase the risk of cardiovascular diseases), and that they know that the risk behaviour is prevalent (phase two; e.g.. know- 
ing that many other people eat too much saturated fat). In the Netherlands, phase two now seems to have been reached. People know that consuming too much fat and too little fruit and vegetables is detrimental to health and they are convinced that many (other) people eat too much fat and too little fruit and vegetables (Riedstra, Hardeman, Pruyn, Aarnink, Van der Feen de Lille, \& Jonkers, 1992). Risk perception research, however, has shown that although people might be convinced of the risk for others, they tend to think of themselves as being less at risk. This is referred to as unrealistic optimism and is often seen in judgments of personal risks (Klein \& Weinstein. 1997: Weinstein, 1988). Such an optimistic bias seems to be prevalent in estimating dietary behaviour as well: many people estimate that they eat less fat and more fruit and vegetables than their peers do. The estimation of 'personal intake compared to others' correlated strongly with self-assessments of 'personal intake', which in turn was found to be the best predictor of intention to change in studies by Brug (Brug \& Lechner, 1995; Brug et al., 1998b). As long as an optimistic bias about personal intake compared to others persists, transition from phase two to phase three will not occur.

In the Precaution Adoption Process Model, personalised feedback on individual risk behaviour (personal feedback) and information about the risk behaviour of others (normative feedback) are considered to be major determinants of the transition from phase two to phase three (Weinstein, 1988). Although the model was originally proposed in the context of precautions against health risks, or external hazards, we think that the model may also apply to the risk behaviours that may cause the actual health risk. Therefore, providing feedback would be an appropriate strategy to enhance awareness of personal risk behaviour and motivate people to change to healthier diets.

Providing personal and normative feedback on dietary habits requires a method that allows one to first assess the personal risk behaviour. Furthermore, since lack of awareness of dietary habits seems to be prevalent among all age groups and all educational levels in the Netherlands (Brug \& Van Assema, 2000) the method should be able to reach large groups of people. In individual counselling, feedback can be personalised to a very high extent, but it cannot reach large groups of people. At the other end of the 'personalisation continuum', mass media interventions can reach large groups of people, but with a minimum of personalised feedback. In the present study we tested two methods of providing feedback, which are somewhere in between these two extremes: computer tafloring and printed self-test forms. The principle of each method is described briefly below, followed by the specific hypotheses of the study.

Computer tailoring is a relatively new technique that has been applied and tested during the last decade for the purpose of influencing various health-related behaviours (Brug et al., 1999; Skinner et al., 1999: Strecher, 1999). Computer-tallored interventions were generally found to be more effective in influencing smoking behaviour (Dijkstra et al. 1998; Strecher, 1999). in encouraging participation in breast cancer screening (Rakowski et al. 1998: Skinner et al. 1994) and in promoting healthy dietary habits (Brug et al. 1999). Computer-tallored information is 
generated by means of three interrelated components: a theory-driven diagnostic tool to diagnose risk behaviour and related determinants, a message library containing feedback messages for all possible diagnoses, and a computer programme that selects, through a set of algorithms, the messages that match the diagnosis (Brug et al. 1999: Kreuter et al., 2000). The set of selected diagnosis-matched messages forms the personalised feedback. The contents of the tailored information depend on the theoretical perspective from which the tailoring programme is developed. For the purpose of influencing awareness of dietary habits, personal and normative feedback on intake levels can be provided. In addition to this, information addressing attitudes, social influence or self-efficacy expectations could be provided to guide people through further stages of change.

The computer taflored interventions that have been tested to date have mainly consisted of printed materials, like letters, newsletters or magazines. The computer-based character of the talloring technique, however, makes it suitable for use in more advanced applications, like interactive programmes on CD-ROM or the Internet. Technically, it is now possible to develop and implement these more advanced computer-tailored interventions, but before disseminating tailored (nutrition) interventions via the World Wide Web, it is important to evaluate the effects of this new application. An interactive web-based computer-tailored nutrition education programme is one of the methods we used to provide feedback in the present study.

Printed self-test forms are the second method we used for providing feedback. This intervention, however much shorter and less individualised than the computer tailored intervention, nevertheless offers the opportunity to provide people with dietary feedback and change information. An important advantage of self-test forms is that they are a relatively easy to develop and distribute tool for health educators in an effort to increase awareness of dietary intake of large groups op people. They have therefore the potential of being used widely in health education practice. This makes it worth-wile to examine the effects of this easy to use and implement feedback intervention next to a more extensive tailored intervention. Printed selftest forms are well-known and popular for their use in lifestyle magazines. These tests allow readers to learn about the 'type' they belong to, as regards, for example, emotional inteiligence or sociability, and what would be the best way for them to act if they belong to a particular type. Self-test forms. like these are partly intended for entertainment, and are therefore relatively easy to use, but they may still be to some extent suitable to classify people into appropriate categories. Self-test forms can be developed for health education topics as well. As such, self-test forms can be found in many self-help manuals, but can also be printed in popular magazines. or used as single forms. Self-test forms consist of a limited number of questions with a multiple-choice answering format. Each answer corresponds with a letter or a number. Summing up the numbers or letters received for each question gives a total score that classifies the respondent into a category. Brief information is then usually provided for each category. The contents of the questions and the information depend on the topic to be addressed in the self-test form. For the 
purpose of influencing awareness of personal intake of fat. fruit and vegetables the information could provide feedback on the assessed intake levels compared to the recommendations. The feedback should be different for each category. The self-test forms tested in this study were developed by the Netherlands Nutrition Centre (Voedingscentrum, 1999), and aimed to increase awareness of dietary habits in large groups of people, by providing dietary feedback and change information. The self-test forms were developed based on the results of studies in the Netherlands showing that lack of awareness was a major barrier towards dietary change le.g. Brug et al., 1994). The self-test forms had not been evaluated on their effects before the present study.

The purposes of the present study were 1) to explore the immediate effects on awareness of and intention to change personal intake of fat, fruit and vegetables of two methods of providing dietary feedback and 2) to study differential effects of the interventions in groups with different levels of education. Differential effects were studied because highly educated people might be more attracted to comprehensive written materials, whereas lower educated people might be more attracted to short and entertaining materials. We conducted a randomised controlled trial, in which the effects of the interventions were compared with those in a control group that received general nutrition information.

We hypothesized that:

- both feedback interventions would have a greater impact on awareness and intention to change than the general nutrition information:

- among the respondents with a higher educational level, the interactive computer-tailored intervention would have a greater impact on awareness and intention to change and would be more appreciated than the self-test forms and the general nutrition information:

- among the respondents with a medium or lower educational level, the self test forms would have a greater impact on awareness and intention to change and would be more appreciated than the computer-tailored intervention and the general nutrition information.

The present study provides information on the process of awareness raising as a prerequisite for dietary change and on the possible value of two feedback interventions in influencing awareness and intention to change intake of fat. fruit and vegetables.

\section{Methods}

\section{Participants}

Participants were 304 students and employees of adult education centres. In line with the demands of the Medical Ethics Committee, general letters explaining the 
purpose and procedure of the study were distributed among a large group of potential participants. Employees could enrol by replying to an e-mail message that was sent out after the letters had been distributed. Students were invited to participate in the study during one of their classes. We emphasised that anybody could enrol, regardless of their interest in nutrition. Recruitment activities were stopped when over 300 respondents had registered for participation. Respondents had to be between 30 and 65 years of age and sufficient understanding of Dutch was required. A $\ell 9$ gift voucher was offered to each participant and a trip to Paris for two was raffled among the participants of the study.

\section{Study design and procedure}

A pre-test-post-test randomised controlled trial with a 3 (two intervention groups and one control group) $\times 2$ (higher versus medium or lower educational level) design was conducted. Respondents from various educational levels were separately assigned to one of the conditions, to ensure an even distribution of educational levels over all three conditions. Two educational levels were distinguished by assigning respondents to either the higher educational level group (university degree or higher professional training) or the medium or lower educational level group (all education below higher professional training).

The experiment was carried out on the site where participants were working or attending their classes, i.e., in offices or classrooms. A maximum of 10 respondents participated in the study simultaneously, but care was taken that they could not see or communicate with each other.

Appointments as to where and when to participate in the study were made with the people who enrolled. When arriving at the agreed location, respondents, who had already been informed about the experiment by means of the letter, were asked to give their informed consent, after which the study procedure was explained in further detail. Lots were drawn to assign each participant to one of the three conditions. Respondents who were assigned to the tailored intervention group started by going through the interactive computer-tailored programme, i.e., filling out the assessment, which was at the same time the baseline questionnaire, and reading the feedback. Respondents with poorly developed computer skills received brief instructions on how to proceed through the programme. Respondents in the self-test form and control groups were first asked to fill out a printed baseline questionnaire. After completion of this questionnaire, self-test forms and printed general nutrition information letters were handed out to respondents in the self-test form and the control group. respectively. Respondents in all three study groups were asked to fill out a printed post-test questionnaire after they had completed and read the intervention materials. Participation in the study ended with filling out this last questionnaire. The whole procedure took 30 to 45 minutes. 
Demographic variables, intake levels of fat, fruit and vegetables and psychosocial variables were assessed using an 84-item self-administered questionnaire. The questionnaire started with assessing age, sex, weight, height, educational level. living situation (alone/together), use of special diets, and whether or not the respondent regularly did the cooking or the shopping for the household. A validated food frequency questionnaire was used to assess intake levels of fat, fruit and vegetables (Van Assema et al., 2001: Van Assema, Brug. Ronda, Steenhuis, \& Oenema, 2002). The first part of the food frequency questionnaire assessed the frequency of use of 19 product categories that contribute most to saturated fat intake, as well as the quantities and types of product (high-fat, medium-fat, or low-fat). The answers to the food frequency questionnaire were used to calculate a fat score, ranging from 0 to 80 , which reflects total saturated fat intake. This short and easy to administer screening instrument allowed us to rank respondents according to individual fat intake, and to detect changes in individual fat consumption (Van Assema et al., 2001). In the second part of the food frequency questionnaire, frequency (how many days a week) and quantities (amount per day) of fruit, fruit juice and vegetable consumption were assessed. This information was used to calculate the average number of servings of fruit and vegetables per day (Van Assema et al., 2002).

Awareness of dietary intake was assessed by measuring realism in self-rated intake compared to others, and in self-rated intake. Respondents were asked to rate their intake compared to others (much lower - much higher) and their personal intake (very low - very high) on five point scales. Measures for 'realism in comparison to others' and 'realism in self-rated intake' were constructed by comparing the self-ratings with objectively assessed intake levels, in terms of below, above or equal to average peer intake levels (peer intake levels were based on data from prior food consumption surveys) and in terms of below, above or equal to recommended intake levels. Respondents whose self-rated intake level corresponded with the objectively assessed intake level were classified as 'realistic', others as 'unrealistic'.

Transtheoretical stages of change were assessed. following the staging algorithm as described by Brug et al. (1997). Attitudes (do you think it is bad or good to eat less fat/more fruit/more vegetables) and self-efficacy (do you think it is difficult or easy to eat less fat/more fruit/more vegetables) were measured on five point scales (very bad - very good: very difficult - very easy) see also. Brug et al. (1998a). These variables were only used to tailor the feedback in the computer-tailored intervention.

The post-test questionnaire differed somewhat in length between the three conditions, because specific questions on attractiveness, usability and experience with self-tests were added in the tailored and self-test groups. Thus, the post-test in the tailored intervention condition had 49 items, that in the self-test condition had 55 and that in the control condition 46. The first part of the questionnaire assessed awareness in the same way as described for the baseline questionnaire. Furthermore, transtheoretical stages of change and general intention to change fat, fruit 
and vegetable consumption (seven-point scales) were assessed. The second part of the questionnaire measured appreciation, relevance, credibility and use of the intervention (see Tables 4.3 and 4.4). These questions were measured on visual analogue scales $(100 \mathrm{~mm})$, anchored by 'completely disagree' and 'completely agree: The questionnaire ended with items about experience and skills in using the computer and the Internet (How often do you use a computer/the Internet? How easy is it for you to use a computer/find something on the Internet?). on sixand five-point scales, respectively.

\section{The tailored intervention}

The components of a computer-tailored education programme are a theory-driven diagnostic tool to assess feedback goals for each participant, a message library containing feedback messages for all possible diagnoses, and a computer programme that selects the feedback messages that correspond with each specific diagnosis (Brug et al., 1999). The whole programme can be provided in printed form or. more advanced, interactively on the computer. In the present study an interactive web-based intervention was used. The intervention was applicable for use on the Internet, but in order to create a controlled situation. we had the programme installed locally on hard disc. The diagnostic tool was the baseline questionnaire described in the previous subsection.

The programme started with a homepage describing what a tailored nutrition education programme is, for whom this particular programme was intended, who had produced it and how it should be used. After reading this homepage, a respondent could start going through the programme by clicking the button for fat, fruit or vegetables in the menu bar on the left side of the screen. Although a random sequence was possible, we asked our respondents to follow the sequence in the menu bar, starting with fat and ending, with fruit. A fourth button gave access to a section with low-fat, high-vegetable recipe suggestions.

Each topic started with a brief general introduction on the subject, after which respondents could click through to the diagnosis section. The questions appeared on the screen one at a time, with the next question appearing as soon as the previous one had been answered. Irrelevant questions, for example questions on meat consumption for respondents who had already indicated that they never ate meat, were skipped. After all relevant questions had been answered, the feedback appeared on the screen, in an attractive layout, with a coloured background and illustrations accompanying the texts. Each feedback section started with a brief introduction and an overview of the topics to be discussed. In the section on fat. feedback was given on how the respondent's computed fat score compared to the recommended intake levels. Respondents who had underestimated their fat intake were confronted with their misconception. and those whose computed fat score exceeded the average fat score of others of the same age and sex were also given feedback on their personal fat score compared to that of others. This information was both written and visualized in a graph. Additional information was given on 
the most important sources of fat in the diet and on ways to replace these products by low-fat alternatives. The information ended with a message that encouraged people in precontemplation to reconsider their fat intake and those in contemplation and preparation to proceed to action, while respondents in action and maintenance were reinforced to continue with their changes. These messages differed for positive. negative or neutral attitudes and positive, negative or neutral self-efficacy expectations. Finally, there were messages on how to succeed in eating low-fat food in possibly difficult situations (when in a restaurant, when eating with one's family or friends, when at a party, when hungry between meals), for those respondents who perceived these situations as difficult.

In the fruit and vegetable sections, feedback was given on the computed intake compared with both the recommendations and the self-rated intake. Respondents who did not meet the recommended levels were given suggestions on how to increase consumption by eating fruit or vegetables either more frequently or in larger portions. Suggestions were also made on how to make it easier to eat fruit and vegetables in the recommended amounts every day. Photographs of recommended portion sizes of three kinds of commonly eaten vegetables were shown in the feedback for respondents who did not meet the recommended intake levels. The information on fruit and vegetable consumption also ended with a message tailored to the respondents' stage of change.

The recipe part included 56 recipes for low-fat, high-vegetable main courses. desserts and snacks, which respondents could browse through. The message library had been tested in earlier studies (Brug et al., 1998a; Brug \& Van Assema, 2000) and was revised and extended for the present study.

A computer programme, written in Visual Basic, enabled the creation and storage of all essential parts of the tailored intervention (screening instrument, message library, tailoring algorithms, and layout of the final feedback). Java script routines generated the tailored messages and created a website consisting of HTML pages. The programme was created for use on the Internet, but could also be used on CD-ROM or hard disk. Internet Explorer 5.0 was the browser used to run the programme. The web-based programme was pre-tested in two sessions and changes were made according to the findings of these pre-tests.

\section{The self-test forms}

The self-test condition involved two printed self-test forms, one for fat and one for fruit and vegetables. The tests used in the present study were developed by the Netherlands Nutrition Centre in collaboration with other Dutch organisations active in the field of health education and promotion. but had never been tested for their effects.

The self-test forms contained a brief introduction on the health benefits of eating either moderate amounts of fat or sufficient amounts of fruit and vegetables. The fat-test form consisted of 14 questions on the frequency with which low- or high-fat foods were consumed. For example, 'when I eat cheese, I eat low-fat cheese', 
with the answering options 'yes', 'no'. 'sometimes'. The low-fat answer would yield the lowest and the high-fat answer the highest score for the question. The self-test forms had a 'scratch card' format. The score on the question could be obtained by scratching open a box that covered a number. After the respondent had scratched open a box for each of the questions, the points that had been received could be added up to a total score with a minimum of 13 and a maximum of 60 points. A total score of less than 22 categorized the respondent in a group with a saturated fat intake in agreement with recommendations. A total score between 22 and 30 categorized the respondent in a group with room for improvement in saturated fat intake. Scores over 30 categorized the respondent in a group of high saturated fat consumers who were strongly recommended to reduce their fat consumption. For each category, feedback was given on how the total fat score (based on the brief assessment) compared with recommendations. In categories two and three, the feedback also referred to general suggestions for reducing fat consumption by either eating fewer products containing fat or replacing high-fat products by low-fat alternatives.

The fruit and vegetables self-test form used six questions to assess the usual frequency and amounts of the respondents' fruit and vegetables consumption. The points that had been received for each question, led to a total score that corresponded with a particular category: 1) sufficient amounts of fruit and vegetables, 2) a certain amount of fruit and vegetables but leaving room for improvements, or 3) insufficient amounts of fruit and vegetables. Each category was given feedback on how the assessed intake compared with recommendations, and referred to general suggestions on how to improve fruit and vegetable consumption, by eating fruit and vegetables with more than one meal and between meals.

The layout of both tests was in bright colours with photographs of low-fat products and fruit and vegetables. The tests had been validated and tested for their appreciation before the start of the present study (Voedingscentrum, 1999).

\section{Contral condition}

Respondents in the control condition received a non-tallored nutrition information letier, with information from brochures by the Netherlands Nutrition Centre. The letter provided information on the importance of a healthy diet and on the fact that Dutch people eat too much fat and too little fruit and vegetables, but that a lot of people do not know they are eating unhealthy diets themselves. The section on fat listed the risks of high-fat diets and emphasised that anybody who eats too much fat is at risk. Low-fat alternatives were given for some high-fat products that are very common in the Dutch diet. The fruit and vegetable part emphasised the positive consequences of eating more fruit and vegetables, followed by suggestions on how to increase fruit and vegetable intake. The letter ended with two low fat recipes. The four-page letter was illustrated with cartoons. 
Binary logistic regression analyses were conducted to test the equality of study groups at baseline. Age, gender, consumption levels of fat, fruit and vegetables, self-rated intake and self-rated intake compared to others were the independent variables in the equations.

The impact of the interventions on awareness (i.e., being realistic) was tested by means of logistic regression analyses, with 'realism in comparison with others' and 'realism in self-rated intake' at post-test as the dependent variables, and study group and the baseline measure of realism as the independent variables. To study group-by-education interaction effects, this interaction term was includied in the logistic regression analyses. Differences in $-2 \log$ likelihood (comparing the fit of a more complex to a more basic model) between the model with the interaction term and the more basic model without this term were computed. In the analyses where the model with interaction was significantly more predictive (i.e., the decrease in $-2 \log$ likelihood was significant), separate analyses were conducted for groups with medium or lower and higher educational levels. Otherwise, the basic model was used.

In logistic regression analysis with three groups, two dummy variables and one reference category are created, thus comparing two groups separately with one reference group. In our study, the control condition was the reference group. However, in order to detect possible differences between the two feedback interventions, additional analyses were conducted, with the self-test condition as the reference group.

Differences between the study groups in the intention to eat less fat and more fruit and vegetables were studied using two-way analyses of variance, with study group and educational level as factors, thus studying intervention and intervention-by-education interaction effects. Tukey was used as the post hoc comparison test.

People with unfavourable diets should profit most from nutrition interventions. In order to study the intervention effects in this specific subgroup the same analyses were repeated for the group of respondents that did not meet the recommended intake levels at baseline.

Differences in subjective impact, use and appreciation of the interventions were studied with two-way analyses of variance and Tukey post hoc comparison tests. The factors in these analyses were study group and educational level.

Differences with $p<.05$ were considered to be significant and differences with $p<.10$ as borderline significant. Analyses were two-tailed. 


\section{Results}

\section{Participants}

A total of 304 respondents participated in the study. The data of 300 respondents were valid for analysis. The information of four respondents in the tailored intervention group was lost due to technical problems. Another four respondents in the tallored intervention group had incomplete data sets; two respondents had completed the whole programme, but the information on fat consumption had not been recorded; two other respondents had only completed the part on fat, because it took them too long to complete the entire programme. For these respondents, the data of the completed and recorded topics were included in the analyses. Analyses. were conducted on 96 subjects in the tailored intervention group. 100 subjects in the self-test group and 102 subjects in the control group.

\section{Baseline measurements}

Mean age of the respondents was 44 years $(S D=9.7)$. Sixty percent of the respondents were female. Almost half of the respondents $(47 \%)$ had a higher educational level (university degree or higher professional training): the other half (53\%) had a medium educational level (medium-level professional training or high school) or a lower educationai ievel (lower-level professional training or primary school).

The average fat score was $18.5(S D=6.4)$ points, the average vegetable intake was 195 grams (2.4 servings, $S D=100$ grams) and the average fruit intake was 1.4 pieces ( 1.4 servings, $S D=1.2$ pieces) per day.

Fifty-four percent of the respondents in the tailored intervention group, $46 \%$ in the self-tesi group and $43 \%$ in the control group had diets that were not in agreement with the recommended fat intake levels. Forty-nine percent, $50 \%$ and $52 \%$ of the respondents in the failored, self-test and control groups, respectively, did not eat 200 grams of vegetables each day, while $47 \%, 51 \%$ and $52 \%$ in the tailored. self-test and control groups, did not meet the recommendation of two pieces of fruit each day.

None of these demographic variables, average intake levels, or key outcome variables differed significantly between the study groups.

\section{Impact on awareness}

Table 4.1 lists the percentages of realistic respondents at baseline and at post-test. In the analyses with 'realism in comparison with others' as the dependent variable, the model with interaction was only found to be significantly more predictive as regards fat intake. Separate analyses of the groups with medium or lower and higher educational levels revealed that only respondents with a medium or lower educational level in the tailored intervention group were significantly more likely to be realistic about their fat intake compared to others (odds ratio $[O R]=11.36$. 
Table 4.1. Percentages of realistic respondents at baseline and post-test

\begin{tabular}{|c|c|c|c|c|c|c|}
\hline & \multicolumn{2}{|c|}{$\begin{array}{l}\text { Tailoring } \\
(n=96)\end{array}$} & \multicolumn{2}{|c|}{$\begin{array}{l}\text { Self-tests } \\
(n-100)\end{array}$} & \multicolumn{2}{|c|}{$\begin{array}{l}\text { Control } \\
(n-102)\end{array}$} \\
\hline & Baseline & Post-test & Baseline & Post-test & Baseline & Post-test \\
\hline \multicolumn{7}{|l|}{ Fat intake } \\
\hline Compared to others & $30 \%$ & $49 \%$ & $45 \%$ & $44 \%$ & $45 \%$ & $39 \%$ \\
\hline Self-rated intake & $28 \%$ & $42 \%$ & $33 \%$ & $35 \%$ & $33 \%$ & $43 \%$ \\
\hline \multicolumn{7}{|l|}{ Vegetable intake } \\
\hline Compared to others & $43 \%$ & $44 \%$ & $52 \%$ & $53 \%$ & $49 \%$ & $49 \%$ \\
\hline Self-rated intake & $42 \%$ & $43 \%$ & $38 \%$ & $41 \%$ & $33 \%$ & $40 \%$ \\
\hline \multicolumn{7}{|l|}{ Fruit intake } \\
\hline Compared to others & $54 \%$ & $60 \%$ & $53 \%$ & $48 \%$ & $53 \%$ & $50 \%$ \\
\hline Self-rated intake & $46 \%$ & $54 \%$ & $53 \%$ & $56 \%$ & $55 \%$ & $57 \%$ \\
\hline
\end{tabular}

$95 \%$ conficence interval $[C I]=3.12-41.31$ ). The basic models revealed a borderline significant effect for 'realism in fruit intake compared to others', with respondents in the tailored intervention group more likely to be realistic $(O R=2.02, C I=0.96$ $-4.23)$.

In the analyses with the self-test group as the reference, respondents in the tailored intervention group were significantly more likely to be realistic about their fat intake compared to others $(O R=2.58, C I=1.14-5.85)$ and about their fruit intake compared to others $(O R=2.19, C I=1.04-4.60)$.

With 'realism in self-rated intake' as the dependent variable, none of the models with interaction were significantly more predictive than the basic model. Respondents in both feedback interventions were no more likely than respondents in the control group to be realistic about their self-rated fat, fruit and vegetable intake. In the analyses with the self-test group as the reference, respondents in the tailored intervention group were significantly more likely to be realistic about their selfrated fat intake $(O R=2.25, C I=1.01-5.09)$.

The subgroup of respondents that did not meet the recommendations showed similar effects for the same groups on the same variables.

\section{Intention to change}

Main effects on intention to change (Table 4.2) in the total study population were found for the intention to eat less fat and more vegetables. Intention to eat less fat was significantly greater in the tailored intervention group than in the two other groups and was greater in the control group than in the self-test group. Intention to eat more vegetables was significantly greater in the tailored intervention group than in the self-test group.

For the subgroup of respondents whose diets were not in agreement with rec- 
Table 4.2. Means and SD for intention to change, F-values for main and group-by-educational level interaction effects and post hoc comparison

\begin{tabular}{|c|c|c|c|c|c|c|}
\hline & \multirow[b]{2}{*}{ Tailoring } & \multirow[b]{2}{*}{ Self-tests } & \multirow[b]{2}{*}{ Control } & \multicolumn{2}{|c|}{ F-value } & \multirow[b]{2}{*}{$\begin{array}{l}\text { Post hoc } \\
\text { comparison }\end{array}$} \\
\hline & & & & Main effect & $\begin{array}{c}\text { Interaction } \\
\text { effect }\end{array}$ & \\
\hline Total study population & $n=96$ & $n=100$ & $n=102$ & & & \\
\hline Intention to eat less fat & $0.72(1.21)$ & $-0.14(1.30)$ & $0.29(1.26)$ & $11.08^{b}$ & 0.60 & $\mathrm{~T}>\mathrm{C}>\mathrm{ST}$ \\
\hline Intention to eat more vegetables & $0.22(1.33)$ & $-0.26(1.21)$ & $0.10(1.21)$ & $3.93^{4}$ & 1.51 & $\mathrm{~T}>\mathrm{ST}$ \\
\hline Intention to eat more fruit & $0.58(1.32)$ & $0.18(1.20)$ & $0.51(1.34)$ & 2,76 & 2.47 & \\
\hline \multicolumn{7}{|c|}{$\begin{array}{l}\text { Group of respondents with diets not in agreement with } \\
\text { recommendations }{ }^{d}\end{array}$} \\
\hline Intention to eat less fat & $0.96(1.15)$ & $0.22(1.15)$ & $0.61(1.20)$ & $4.05^{4}$ & 0.54 & $\mathrm{~T}>\mathrm{ST}$ \\
\hline Intention to eat more vegetables & $0.64(1.15)$ & $-0.10(1.25)$ & $0.15(1.23)$ & $5.08^{\mathrm{b}}$ & 2.09 & $\mathrm{~T}>\mathrm{ST}$ \\
\hline Intention to eat more fruit & $1.27(1.01)$ & $0.53(1.19)$ & $0.75(1.27)$ & $5.31^{\mathrm{b}}$ & 2.10 & $\mathrm{~T}>\mathrm{ST}, \mathrm{C}$ \\
\hline
\end{tabular}

Note. ${ }^{\mathrm{A}} p<.05,{ }^{\mathrm{b}} p<.01,{ }^{\mathrm{c}} p<.10,{ }^{\mathrm{d}}$ Numbers of respondents in each sub-group can be derived from the percentages presented in the baseline measurement section. 
ommendations, main effects were found for the intention to eat less fat, more vegetables and more fruit. Respondents in the tailored intervention group had a greater intention to change than respondents in the self-test group. For fruit, there was an additional borderline significant $(p=.08)$ difference in intention to change between the tailored intervention and control groups. There were no interventionby-education interaction effects for any of the intention items.

\section{Subjective impact, use and appreciation}

The subjective impact and usability measures (Table 4.3) only revealed main effects. Respondents in the tailored intervention group stated significantly more often than respondents in the two other groups that they, as a result of the intervention. had changed their opinion about their dietary habits $[F(2,290)=9.7, p=, 000 \mid$ and intended to change their diet $[F(2,291)=13.4, p=.000]$. Respondents in all three groups rated the information equally attractive to read, but the information in the self-test forms was read significantly less extensively than the information in the control group $[F(2,290]=4.3, p=.015]$. The tailored intervention programme was significantly more likely to be used again than the self-test forms and the nutrition information letter $[F(2,288] \mid=4.3, p=.014]$.

Of all items assessing appreciation of the intervention materials (see Table 4.4), a significant interaction effect was only found for newness of the information on fat and vegetables. Separate analyses for the groups with higher and medium or lower educational levels revealed that only the respondents with a medium or lower educational level in the taflored intervention group rated the information on fat and vegetables as more new to them than respondents in the two other conditions [for fat: $F(2,151]=12.8, p=.000$; for vegetables: $F 2,150$ ) $=10.5, p=.0001$. As regards the newness of the information on fruit, only a main effect was found, with the information provided in the tailored intervention rated as more new than the

Table 4.3. Subjective impact and use of the nutrition information in the three conditions (means, standard deviation and post hoc comparison)

\begin{tabular}{|c|c|c|c|c|}
\hline & $\begin{array}{l}\text { Tailored } \\
(n-96)\end{array}$ & $\begin{array}{l}\text { Self-test } \\
(n-100)\end{array}$ & $\begin{array}{l}\text { Control } \\
(n-101)\end{array}$ & Post hoc \\
\hline $\begin{array}{l}\text { Changed opinion about diet as a } \\
\text { result of the information }\end{array}$ & $59.8(28.5)$ & $42.5(31.5)$ & $43.1(32.9)$ & $\mathrm{T}>\mathrm{ST}, \mathrm{C}$ \\
\hline $\begin{array}{l}\text { Intend to change diet as a result of the } \\
\text { information }\end{array}$ & $64.8(28.6)$ & $42.4(31.6)$ & $50.4(31.8)$ & $\mathrm{T}>\mathrm{ST}, \mathrm{C}$ \\
\hline How much of the information read & $93.0(11.6)$ & $89.1(18.4)$ & $94.9(11.9)$ & $\mathrm{C}>\mathrm{ST}$ \\
\hline $\begin{array}{l}\text { Use the programme/read the informa- } \\
\text { tion again when possible }\end{array}$ & $70.3(32.5)$ & $57.3(33.0)$ & $58.2(36.4)$ & $\mathrm{T}>\mathrm{ST}, \mathrm{C}$ \\
\hline Altractiveness of information & $85.0(16.7)$ & $81.3(22.3)$ & $80.5(22.1)$ & \\
\hline
\end{tabular}




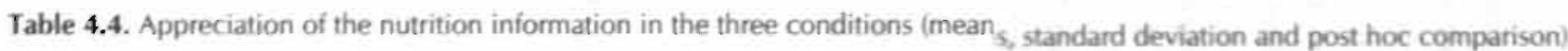

\begin{tabular}{|c|c|c|c|c|c|c|c|c|c|c|c|c|}
\hline & \multicolumn{4}{|c|}{ For fat } & \multicolumn{4}{|c|}{ For vegetables } & \multicolumn{4}{|c|}{ For fruit } \\
\hline & $\begin{array}{l}\text { Tailored } \\
(n=95)\end{array}$ & $\begin{array}{l}\text { Self-test } \\
(n-99)\end{array}$ & $\begin{array}{c}\text { Control } \\
(n-101)\end{array}$ & Post hoc & $\begin{array}{l}\text { Tailored } \\
(n-95)\end{array}$ & $\begin{array}{l}\text { Styf-test } \\
(n)=99)\end{array}$ & $\begin{array}{l}\text { Control } \\
\text { (n - 101) }\end{array}$ & Post hoc & $\begin{array}{l}\text { Tailored } \\
(n-94)\end{array}$ & $\begin{array}{l}\text { Self-test } \\
\text { (n - 98) }\end{array}$ & $\begin{array}{l}\text { Control } \\
(n-101)\end{array}$ & Post hoc \\
\hline $\begin{array}{l}\text { The information was } \\
\text { understandable }\end{array}$ & $\begin{array}{l}89.2 \\
(16.4)\end{array}$ & $\begin{array}{l}89.7 \\
(13.0)\end{array}$ & $\begin{array}{l}92.3 \\
(12.0)\end{array}$ & & $\begin{array}{l}90.7 \\
(11.9)\end{array}$ & $\begin{array}{l}91.4 \\
(10.4)\end{array}$ & $\begin{array}{l}91.3 \\
(13.3)\end{array}$ & & $\begin{array}{l}91.7 \\
(11.0)\end{array}$ & $\begin{array}{r}90.4 \\
(12.0)\end{array}$ & $\begin{array}{r}91.9 \\
(13.2)\end{array}$ & \\
\hline $\begin{array}{l}\text { The information was } \\
\text { interesting }\end{array}$ & $\begin{array}{l}78.2 \\
(22.2)\end{array}$ & $\begin{array}{l}73.0 \\
(24.7)\end{array}$ & $\begin{array}{l}74.5 \\
(25.3)\end{array}$ & & $\begin{array}{l}73.3 \\
(26.5)\end{array}$ & $\frac{58.8}{(28.2)}$ & $\begin{array}{l}75.3 \\
(25.5)\end{array}$ & & $\begin{array}{c}75.5 \\
(25.1)\end{array}$ & $\begin{array}{c}69.3 \\
(27.5)\end{array}$ & $\begin{array}{l}77.0 \\
(24.1)\end{array}$ & \\
\hline $\begin{array}{l}\text { The information given } \\
\text { was credible }\end{array}$ & $\begin{array}{l}85.1 \\
(21.4)\end{array}$ & $\begin{array}{l}85.1 \\
(16.9)\end{array}$ & $\begin{array}{c}88.0 \\
(18.8)\end{array}$ & & $\begin{array}{l}87.6 \\
(14.6)\end{array}$ & $\begin{array}{l}85.0 \\
(17.1)\end{array}$ & $\begin{array}{l}90.3 \\
(15.6)\end{array}$ & & $\begin{array}{l}87.8 \\
{[16.4]}\end{array}$ & $\begin{array}{l}85.6 \\
(16.1)\end{array}$ & $\begin{array}{l}89.1 \\
(16.2)\end{array}$ & \\
\hline $\begin{array}{l}\text { The information was } \\
\text { new to me }\end{array}$ & $\begin{array}{c}38.1 \\
(34.3)\end{array}$ & $\begin{array}{l}21.2 \\
(25.6)\end{array}$ & $\begin{array}{c}18.2 \\
(25.2)\end{array}$ & $\mathrm{T}>\mathrm{ST}, \mathrm{C}^{\prime}$ & $\begin{array}{l}33.2 \\
(32.0)\end{array}$ & $\begin{array}{l}21.3 \\
26.0)\end{array}$ & $\begin{array}{l}17.0 \\
(23.2)\end{array}$ & $\mathrm{T}>\mathrm{ST}, \mathrm{C}^{\circ}$ & $\begin{array}{l}31.5 \\
(30.5)\end{array}$ & $\begin{array}{l}21.9 \\
(25.4)\end{array}$ & $\begin{array}{l}16.7 \\
(22.9)\end{array}$ & $\mathrm{T}>\mathrm{ST}, \mathrm{C}$ \\
\hline $\begin{array}{l}\text { The information was } \\
\text { of personal relevance } \\
\text { to me }\end{array}$ & $\begin{array}{r}75.1 \\
(23.7)\end{array}$ & $\begin{array}{l}65.1 \\
(29.2)\end{array}$ & $\begin{array}{r}60.6 \\
(33.7)\end{array}$ & $T>S T, C$ & $\begin{array}{c}70.5 \\
(26.6)\end{array}$ & $\begin{array}{r}59.3 \\
(31.5)\end{array}$ & $\begin{array}{l}55.9 \\
(35.7)\end{array}$ & $\mathrm{T}>\mathrm{ST}, \mathrm{C}$ & $\begin{array}{l}71.8 \\
(26.5)\end{array}$ & $\begin{array}{l}61.2 \\
(32.5)\end{array}$ & $\begin{array}{l}63.5 \\
(34.4)\end{array}$ & $\mathrm{T}>\mathrm{ST}$ \\
\hline $\begin{array}{l}\text { The information was } \\
\text { individualised }\end{array}$ & $\begin{array}{l}74.2 \\
(26.2) \\
\end{array}$ & $\begin{array}{r}51: 6 \\
(31.7)\end{array}$ & $\begin{array}{r}45.2 \\
(32.3)\end{array}$ & $\mathrm{T}>\mathrm{ST}, \mathrm{C}$ & $\begin{array}{c}69.5 \\
(28.0) \\
\end{array}$ & $\begin{array}{r}51.9 \\
(31.9)\end{array}$ & $\begin{array}{r}39.8 \\
(33.4)\end{array}$ & $\mathrm{T}>\mathrm{ST}>\mathrm{C}$ & $\begin{array}{c}70.4 \\
(27.5)\end{array}$ & $\begin{array}{l}49.6 \\
(32.3)\end{array}$ & $\begin{array}{l}48.7 \\
(34.1)\end{array}$ & $\mathrm{T}>\mathrm{ST}, \mathrm{C}$ \\
\hline
\end{tabular}

Note. "These post hoc differences only apply to the groups with medium or fowe, level of education. All scales range from 0 (completely disagree) - 100 (completely agree). 
information in the other two groups $[F 2,288]=7.6, p=.001]$.

All study groups and educational levels rated the information on all three topics as equally credible, interesting and comprehensible. Main effects were found for the personal relevance of the information (for fat: $F(2,288$ ) $=6.1, p=.002$; for vegetables: $F 2,288)=5.7, p=.004$; for fruit: $F[2,287)=2.9, p=.054]$ and for the extent to which the information was individualised [for fat: $F(2,289$ ) $=23.7$. $p=.000 ;$ for vegetables: $F(2,289)=21.9, p=.000$; for fruit: $F(2,287)=14.2, p=$ .0001 . The information provided to the tallored intervention group was perceived as being of greater personal relevance and more individualised than the information provided to the two other groups. The information on vegetables was rated as more personally relevant in the self-test condition than in the control condition.

\section{Discussion}

The results of the present study, in which we investigated the immediate impact of two different methods of providing people with feedback about their fat, fruit and vegetable consumption, indicate that only the interactive computer-tailored nutrition education programme had a greater immediate impact on awareness and intention to change than the general nutrition information. Further, the tailored intervention was rated as more effective by respondents and was appreciated better than the control intervention. The hypothesised effects on awareness and intention to change for the self-test form intervention were not found. The additional analyses in which the two feedback interventions were compared did not show results in favour of the self-test forms either. The tailored intervention resulted in significantly more realism about the respondents' own dietary intake, a greater intention to change, more subjective impact and greater appreciation than the self-test form intervention.

The hypotheses that the tailored intervention would outperform the self-test form intervention only in the group with a higher educational level, whereas the reverse was expected for the group with a medium or lower educational level, were not supported by the data either. The few differences in intervention effects for different levels of education indicated that the respondents with a medium or lower educational level may have benefited more from the tailored intervention. This is in line with recent findings by Brug \& Van Assema (2000). It appears that extensive written materials, even in a digital form, appeal to people with a medium or lower educational level as well. It must be noted, however, that all participants in our study had a certain level of education. The results are not applicable to groups with no formal education at all.

Although we found some effects of the tailored intervention on awareness variables, the impact was limited to an increase in 'realism in comparison with others'. Based on the theoretical framework, we expected that the personal and normative feedback would have resulted in more realism in both awareness variables. The fact that the intervention caused more realism in comparison with others may in- 
dicate a first step toward a more realistic self-rated intake, but the next step, actually becoming more realistic in self-rated intake, may take more time. Whether or not this argument holds will have to be investigated in studies of the longer-term effects of feedback on awareness of personal intake levels.

The tallored intervention seemed to be most effective in influencing awareness variables and intention to change for fat intake, whereas effects on awareness and intention to change for fruit and vegetables were hardly found. These findings are in line with earlier studies that found tailored interventions to be more effective in changing (cognittions related to) dietary fat consumption than in changing fruit and vegetable intake (Brug et al, 1999). Possible explanations for the absence of an impact on awareness and intention to change for fruit and vegetables in this study are that at baseline respondents seemed already more realistic about their fruit and vegetable than about their fat intake. This higher level of baseline awareness may make it more difficult to accomplish changes by raising awareness. This higher baseline awareness level may also indicate that lack of awareness as a barrier toward motivation to change is less prominent for fruit and vegetable consumption than it is for fat consumption, which may explain the absence of an impact on intention to change. Other explanations may be found in the intervention itself, or in the sequence in which we asked the respondents to use the tailored information. The information about fruit and vegetables was not as extensive as the information on fat consumption. Furthermore, we asked respondents to start the tailored intervention programme with the information on fat consumption. This sequence may have resulted in a more intensive processing of the information on fat than that on vegetables and fruit. In future studies the information provided on fruit and vegetable consumption may have to be extended and the effect of sequence in which the information is used should be explored.

The subjectively perceived and reported effects of the tailored intervention were significantly better than those of the interventions in the two other groups. Furthermore, the tailored intervention was appreciated better on the important features of effective nutrition interventions (Contento et al., 1995), viz., personal relevance, individualisation and novelty of information. This finding is consistent with those of previous studies on computer-tailored nutrition education (e.g., Brug et al., 1996: Campbell et al., 1994). It is promising that these positive subjective and appreciation effects have now also been found for an interactive web-based computer-tailored intervention.

Even though there seem to be some similarities, the comparability of the results of the present study with those of previous studies is limited, because most studies have measured short-term ( 1 month) or longer-term (> 4 months) effects, and have mainly focused on behavioural change (Brug et al., 1999: Campbell et al., 1994) rather than on immediate effects on pre-behavioural determinants. Furthermore, only one earlier study assessed effects of tailored information on awareness as an important determinant of motivation to change behaviour (De Bourdeaudhuij \& Brug. 2000). Finally, the results of the present study are not readily comparable with those of other studies because the tailored interventions that have been re- 
ported to date have mainly been printed versions. The number of published studies into the effects of more advanced interactive and multimedia tailored interventions is now growing (e.g., Campbell et al., 1999; Oenema et al., 2001), but is still too small to make valid comparisons.

The tailored intervention is developed both for people who do and for those who do not have diets in agreement with recommendations, but the latter group should benefit most from the intervention. In our study, however, there was no greater impact in the group of people with unfavourable diets. A possible explanation for this comes from the body of literature on feedback (e.g., DiClemente, Marinilli. Singh. \& Bellino, 2001; Kluger \& DeNisi, 1996; Weinstein \& Klein, 1995) from which it becomes clear that the working mechanism of feedback on behavioural change is not always straightforward, especially not among people who receive unexpected feedback information. Studies on dietary feedback by Bowen (Bowen, Fries, \& Hopp, 1994). Brug et al. (1998a) and Van Assema (Van Assema, Brug. Dolders. Kok, \& Steenhuis, 1996) for example found that respondents in high-risk groups downgraded the accuracy, seriousness or importance of the feedback information. Processes like these, or other, unknown processes, may account for the limited results of the tailored intervention, especially in the subgroup of respondents with diets that are not in agreement with recommendations. Improved understanding of the responses to feedback and the underlying processes of feedback may make it possible to more specifically address groups at high risk and increase the impact of feedback information.

The lack of an effect of the self-test form intervention may be attributable to the fact that the feedback provided was too brief, too general and not seen as personally relevant or individualised. Thus, the self-test forms seem to lack the important features of successful nutrition education interventions. Additional research is needed to gain more insight into why this self-test form intervention failed to have an impact.

There were some limitations to the present study. Even though we tried to avoid selecting a sample of respondents who were already greatly interested in food and nutrition by emphasising that anybody could enrol in the study, we still had a self-selected sample of respondents who may have been interested in nutrition more than average. The fact that our sample included a higher proportion of respondents with diets in agreement with recommendations than the general Dutch population (Voedingscentrum, 1998) may reflect this. However, respondents with fat. fruit and vegetable intakes not in line with recommended intake levels were well represented in the study sample and all analyses were conducted separately for these respondents. These separate analyses showed similar results. Therefore. although the study sample may not have been representative for the entire Dutch adult population, the results of the present study also hold for people with less favourable diets. The interventions were tested in a highly controlled situation, which enhanced internal validity but probably lowered external validity. In a reallife setting, respondents could easily be distracted from properly using the talloring programme or filling out the self-test forms. which might lead to different effects. 
Furthermore, an electronic questionnaire was used as the baseline questionnaire in the tallored intervention group, whereas printed questionnaires were used in the two other groups. Studies comparing web-based questionnaires with paper-andpencil questionnaires did not find differences between the two (e.g., Davis, 1999). Although we cannot rule out that the different methods of data collection had some effect in our study, we did not find any differences in intake levels, or other key variables between the study groups at baseline. Since equal groups could be expected because of the randomisation procedure it seems that the different baseline data collection methods did not have a relevant effect on the results. Finally, although the way we conducted this randomised controlled trial allowed us to study immediate effects in a controlled situation, which was what we aimed for, we do lack information on long-term effects and effects on behaviour. The results should therefore be interpreted with caution.

The present study is one of very few that provide information on the immediate impact of two different feedback interventions on pre-behavioural determinants, as well as the first to specifically assess immediate impact on awareness of personal behaviour. It demonstrates that an intervention can exert an effect immediately after exposure. In this study we only found an immediate impact for the interactive computer-tailored intervention and not for the self-test form intervention. This leads us to conclude that the interactive computer-tailored intervention is a more promising tool for inducing important steps toward behaviour change. Future studies should aim at testing the longer-term effects of the interactive computertallored intervention on awareness, intention and behaviour, and the possibilities of implementing and disseminating this intervention via the World Wide Web. 


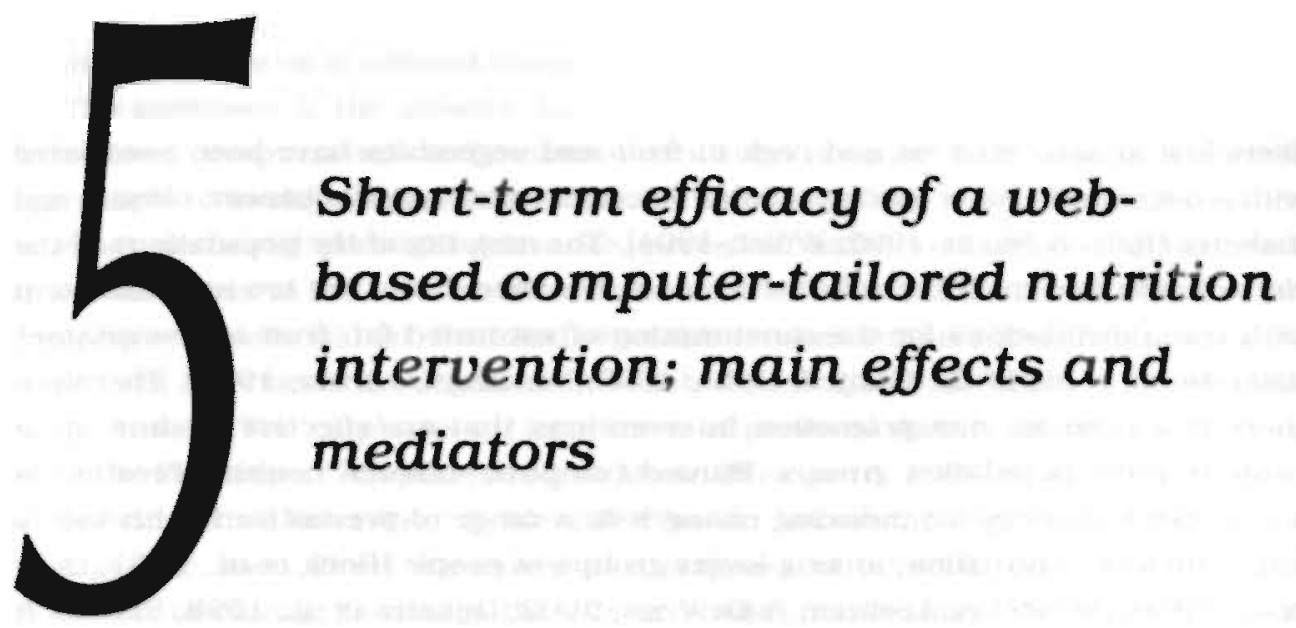

Abstract

This study evaluates the short-term efficacy and respondents' evaluations of a web-based computer-tailored nutrition intervention, aiming to decrease saturated fat intake and increase fruit and vegetable intake. Perceived personal relevance. individualisation and interestingness of the information were tested as mediators of the effects of the tailored intervention. Respondents $(N=782)$ were randomly assigned to a tailored intervention group, a generic nutrition information control group or a no information control group. Fat, fruit and vegetable intakes and behavioural determinants were measured at baseline and at three weeks post-intervention. Post-test group differences were determined by multiple linear regression analyses. The computer-tailored intervention produced significant effects for the determinants of fat, fruit and vegetable intake and for vegetable and fruit intake. The tailored information was rated as more personally relevant, individualised. interesting and new than the generic nutrition information. Perceived personal relevance, individualisation and interestingness were identified as mediators of some of the tailoring effects. The findings of the present study indicate that web-based. computer-tailored nutrition information can have a short-term effect on the determinants of fat, fruit and vegetable intake. The effect of the tailored information may be partly explained by the perceived personal relevance and individualisation of the information.

Accepted for publication as: Oenema, A., Tan, F., \& Brug, J. Short-term efficacy of a web-based computer-tailored nutrition intervention; main effects and mediators. Annals of Behavioral Medicine. 


\section{Introduction}

Diets low in saturated fat and high in fruit and vegetables have been associated with a decreased risk of cardiovascular diseases, some types of cancer, obesity and diabetes (Rolls \& Shide, 1992; Willet, 1994). The majority of the populations of the Netherlands and most other Western countries have diets that are not consistent with recommendations for the consumption of saturated fat, fruit and vegetables (Beer-Borst et al, 2000; Ganji \& Betts, 1995; Voedingscentrum, 1998). Therefore, there is a need for diet promotion interventions that are effective for and applicable to large population groups. Printed computer-tallored health education is a promising strategy for inducing changes in a range of preventive health behaviours, including nutrition, among larger groups of people (Bock et al., 2001; Brug et al., 1999: De Nooijer, Lechner, \& De Vries, 2002; Dijkstra et al., 1998; Kreuter \& Strecher, 1996: Rakowski et al., 1998: Skinner et al., 1999: Strecher, 1999). More advanced interactive, multi-media and Internet applications of computer-tailored interventions are now feasible, and may have great potential for tailored nutrition education. An Internet-based intervention may potentially reach even larger groups of people, may provide more accurately tailored information because the time span between the assessment and the provision of tailored information is reduced to a minimum, and requires less human effort for distribution than the more traditional printed interventions (Brug et al., 2003; Oenema et al., 2001: Strecher et al. 1999). Although tailored nutrition interventions that use interactive multimedia or Internet-based systems to deliver the information are increasingly becoming available, few of these interventions have been formally evaluated (Anderson et al., 2001: Brug et al., 2003: Campbell et al., 1999).

The mechanisms through which tailored interventions exert their effects require further investigation (Abrams et al., 1999; De Vries \& Brug, 1999; Kreuter et al. 1999; Skinner et al., 1999; Strecher, 1999). It has been suggested that tailored interventions may contain less redundant information, focus people better on the health message, and lead to more personal involvement in the health message (De Vries \& Brug, 1999). In research to date, tailored nutrition interventions have been found to be perceived as more personally relevant, more individualised, more interesting and more likely to be read completely than non-tailored materials (Brug et al., 1999: De Bourdeaudhuij \& Brug. 2000). Greater perceived personal relevance, individualisation and interestingness of the information may therefore be of influence to the effects of tailored information. According to the Elaboration Likelihood Model (Petty \& Cacioppo, 1986) information can be processed through a central and a periferal route. Information processing through the central route is more thoughtful and effortful, and therefore more likely to result in stronger and more enduring changes in attitude, intention and behaviour. Motivation to process information is an important condition for central route information processing. Motivation is induced by personal relevance of the information and may also be induced by individualisation and interestingness (Petty et al., 2002: Petty \& Cacioppo, 1986). It has, however, not been tested whether these factors actually do 
mediate the effects of tailored interventions.

The purposes of the present study were (1) to test the short-term impact of a web-based computer-tailored nutrition education programme that aims to encourage people to modify their saturated fat, fruit and vegetable intakes and (2) to study whether perceived personal relevance, perceived individualisation and perceived interestingness of the information mediate the effects of the tailored intervention. Awareness of personal fat, fruit and vegetable intakes and intention to change were important intermediate outcomes on which the effects of the tailored intervention were studied. The emphasis on awareness of personal dietary intake was derived from the Precaution Adoption Process Model (Weinstein, 1988) in which awareness of personal risk behaviour is identified as an important step toward behaviour change. We used direct measures of awareness of personal dietary intake and intention to change to assess the effects of the intervention. The study was conducted as a randomised trial with three study groups, comparing the tailored in. tervention with a generic nutrition information control group and a no information control group. The tailored intervention was expected to produce greater changes in the outcome measures than the generic information control group and the no information control group. Furthermore, the tailored information was expected to be perceived as more personally relevant. more individualised and more interesting than the generic nutrition information, and these attributes were expected to mediate the effects on awareness, intentions and behaviours.

\section{Methods}

\section{Participants}

Of the 2133 people that were invited to participate in the study, $904(42 \%)$ responded by returning the baseline questionnaire. Eight hundred and fifteen (90\%) of these responders indicated that they were willing to participate in the study. Thirty-three $(4 \%)$ responders who were willing to participate in the study did not meet the inclusion criterion of having access to a suitable computer. The remaining. 782 respondents were randomly assigned to one of the conditions (tailored intervention group $n=261$; generic information control group $n=260$ : no information control group $n=261$ ). Of the initial sample, $616(79 \%)$ respondents returned the post-test questionnaire: $188(72 \%)$ in the tailored intervention. $196.75 \%)$ in the generic information and $232(89 \%)$ in the no information group.

\section{Procedure}

Participants were recruited via seven employers in the southern part of the Netherlands. The settings included factories. institutes for health and social care and a local government institute. Announcements of the study were published in inhouse newsletters approximately two weeks prior to its commencement. In line 
with the requirements of the Medical Ethics Committee, the goal and procedure of the study were explained in a personal invitation letter that was sent to employees at the start of the study, accompanied by a baseline questionnaire. Respondents could enrol in the study by expressing their consent for participation on the first page of the baseline questionnaire and by completing this questionnaire. Some of the employers allowed their employees to participate in the study during working hours. Respondents had to be between 18 to 65 years of age, have a sufficient command of Dutch and have access to a personal computer with a CD-ROM drive, either at work or at home.

Eligible respondents were randomly assigned to one of the three conditions. Four weeks after the baseline questionnaire had been returned, respondents in the tallored and generic information groups received a CD-ROM with the assigned programme, or a letter explaining how to get access to the assigned programme on the work-site Intranet. Respondents in the no information control group received a letter that confirmed their participation to the study and explained that they would receive the next questionnaire in three weeks. Three weeks after the intervention materials had been sent, the post-test questionnaires were mailed to the participants. Respondents who did not return the post-test questionnaire within the requested time were sent a reminder letter. As an incentive for participation. respondents could win one of twenty $\ell 20$ gift vouchers that were raffled among participants who completed all parts of the study.

\section{Tailored intervention}

Generating and delivering a computer-tailored health intervention requires a theory-driven diagnostic tool, a database that stores the data obtained with this diagnostic tool, a database that stores messages corresponding with all possible diagnoses, a computer programme that links up each possible diagnosis with the appropriate message and a channel through which the selected set of messages is presented [Brug et al., 1999: Brug et al., 2003: Kreuter et al., 2000).

The diagnostic tool used to generate the computer-tailored programme in this study was similar to the baseline questionnaire as described in the measurement section, with additional assessments of attitides and self-efficacy expectations. Since the programme aimed to raise awareness of personal dietary intake, the message database included messages providing feedback about personal intake levels of fat, fruit and vegetables and how these intake levels compared with recommendations and the average intake levels of peers (Oenema \& Brug. 2003; Weinstein, 1988). Subsequent messages encouraged participants to consider making changes to their diet, to actually start making changes, or to maintain current eating patterns, tailored to their diagnosed intake levels and motivation to change. In order to increase participants' self-efficacy, information was provided about what changes to make and on how to make these changes. This information was tailored to the dietary choices people had reported in the diagnostic tool. Respondents who indicated that they were willing to change their fruit and vegetable intake were en- 
couraged to form implementation intentions, i.e., make specific action plans about where and when to eat fruit and vegetables (Orbell \& Sheeran, 2002). Information was given on how to make implementation intentions and sex- and age-tailored role model stories were provided to illustrate how implementation intentions could work. Respondents were also able to browse through an extensive list of low saturated fat, high vegetable recipe suggestions. The tailored intervention was an adapted version of the intervention that has been described in more detail elsewhere (Oenema et al., 2001).

The diagnostic tool had to be completed as an electronic questionnaire on a personal computer. Immediately after respondents had answered the questions about each topic (fat, fruit or vegetables) the tailored information relating to that topic emerged on the screen. The respondents could choose in which order to go through the topics. The information could be read directly from the screen or a print-out could be made. The intervention consisted of HTML pages, linked together to form a web-site. The web-site was developed for use on the Internet, but in order to create a controlled situation in which the sole impact of the programme could be studied, it was administered locally. Some of the respondents received the programme on a CD-ROM to be used on their PC at home or at work, while others had access to the programme through the work-site Intranet. The programme was run using the Internet Explorer 5.0 browser. Respondents could use the programme as often as they liked.

\section{Generic nutrition information}

The generic nutrition information was also delivered in a web-based form, and comprised information from standard brochures published by the Netherlands Nutrition Centre. The information stressed the health benefits of a low fat and high fruit and vegetable intake, and the fact that many people consume diets high in fat or low in fruit and vegetables, without being aware of this. The information emphasised that anybody, including the reader, could have a diet high in fat or low in fruit and vegetables and that they might consider making changes to their intakes. General suggestions were given on how to lower saturated fat intake or increase fruit and vegetable intake, based on common Dutch consumption patterns. The same list of recipe suggestions as in the tailored intervention was provided. The generic nutrition information was provided as a web-site consisting of HTML pages. with the same layout as the tailored intervention, to make the tailored and generic nutrition information programme appear as similar as possible. The programme was provided either on CD-ROM or through the work-site Intranet.

\section{Measurements}

A measurement instrument was developed, through which it was possible to measure awareness of personal intake levels, intention to change and actual intake of saturated fat, fruit and vegetables. Fat, fruit and vegetable intakes were measured 
at baseline and follow-up by means of validated food frequency questionnaires (Bogers, Van Assema, Kester, Westerterp, \& Dagnelie, accepted; Van Assema et al., 2001: Van Assema et al., 2002). The 35-item questionnaire for fat assessed the intake of food products that contribute most to saturated fat intake in the Netherlands (i.e., dairy products, bread spreads, cheese, meat, gravy. sweet snacks and savoury snacks). Answers to the items were converted into a fat score ranging from 0 to 80 , reflecting total saturated fat intake. The 14-item fruit and vegetable questionnaire assessed the intake of raw and cooked vegetables, fruit and fruit juice. Average daily intake of servings of vegetables and fruit were computed. Self-rated intake and intention to change were assessed at baseline and follow up, using single items for each behaviour [how much fat/fruit/vegetables do you think you eat (very little - very much); do you intend to eat less fat/more fruit/more vegetables (certainly do not - certainly do)]. Answering formats were five-point scales. These measures have been used in previous studies examining the relation between objectively and subjectively assessed intakes of fat, fruit and vegetables (Brug et al. 1994: Glanz et al., 1997; Lechner et al., 1997). Self-rated intake can be used as an indicator for awareness of personal dietary intake, by combining this measure with objectively assessed intake (Brug et al. 1998b). In the present study self-rated intake was combined with objectively assessed intake by statistically adjusting for objectively assessed intake in the analyses on self-rated intake. Demographic and general information such as height, weight, gender, age, educational level, ethnic background and household composition were obtained from the participants at baseline.

The post-test questionnaire for the respondents in both of the nutrition information groups contained an additional 21 items evaluating the nutrition information programme (Table 5.1). Questions about the programme included whether the information was attractive (very unattractive - very attractive), whether the programme was user-friendly (very difficult - very easy to use), whether receiving the information via the programme's delivery mode was enjoyable fvery unenjoyable - very enjoyable), whether the information was easy or difficult to understand (very difficult - very easy), whether the information was interesting (very uninteresting - very interesting) and how much of the information was read (none of it - all of it). Answering formats were seven-point scales. Specific questions about the fat, iruit and vegefable information assessed how individualised, personally relevant. new, credible and usable the information on each of these topics was perceived to be, through statements on seven-point scales (completely disagree - completely agree). Furthermore. it was assessed how often people had used the information programme and how much time on average they spent using the programme. The questions in this part of the questionnaire were adapted from the process questionnaire as proposed by Brug and colleagues (Brug et al., 1996).

\section{Statisțical analyșes}

Logistic regression analyses with drop out (yes/no) between baseline and post-test 
as the dependent variable, and study group. gender, age, educational level, body mass index (BMI), and baseline measures of self-rated intake, intention to change and intake levels of fat. fruit or vegetables as the independent variables, were conducted to identify predictors of drop out. Further logistic regression analyses were conducted with the study group as the dependent variable and age, gender, educational level, BMI. and baseline measures of self-rated intake, intention to change and intake levels of fat, fruit or vegetables as the independent variables, to examine the similarity of the final study groups at baseline.

The hypotheses of the present study were tested using analyses of variance and multiple linear regression analyses. The analyses were conducted for the total study population and then repeated for subgroups of respondents with baseline dietary intake levels not in agreement with recommendations (at risk) and respondents who at baseline were unaware of their high fat, or low fruit and vegetable intake (unaware). The subgroup analyses were performed since these groups should profit most from the intervention. Analyses of variance were used to study differences in evaluations and use of the tailored and the generic nutrition information. The variables included in these analyses are listed in Table 5.1. Multiple linear regression analyses were conducted to test for post-test group differences in outcome measures. For these multiple regression analyses, group was dummy coded with the tailored intervention group as the reference, since the purpose of the study was to compare the tailored intervention group with both other groups. Analyses were conducted with the post-test score of each single outcome indicator (i.e., selfrated intake, intention and objectively assessed intake) as the dependent variable and the dummies for group and the baseline score for that outcome indicator as the independent variables. The analyses on self-rated intake included the post-test intake measure as an additional independent variable, to create the indicator for awareness. These analyses were conducted among the responders to the post-test. and repeated for all participants at baseline in an intention-to-treat procedure.

Sets of three regression equations were used to test perceived personal relevance, individualisation and interestingness of the information as mediators, following the strategy of mediation analysis suggested by Baron \& Kenny (1986). For these analyses group was dummy coded into a treatment group variable, as one for the tailored information group and zero for the generic information group. In the first equation, each hypothesised mediator was regressed on the treatment group variable and the independent variables that were included in the multiple regression analyses on the outcome measures. In the second equation. the outcome measure was regressed on the treatment group variable and the above-mentioned independent variables. In the third equation, the dependent variable was regressed on the treatment group variable, the above-mentioned independent variables and the mediator. A variable can be identified as a mediator if the treatment group. variable affects the mediator in the first equation, the treatment group variable affects the dependent variable in the second equation and the mediator affects the dependent variable in the third equation. while the effect of the treatment group variable on the dependent variable has become significantly smaller than in the 
second equation. If the effect of the treatment group variable on the dependent variable is no longer significant when the mediator is controlled, mediation is complete (Baron \& Kenny, 1986). Significance of mediation was tested by calculating a $z$-score and $p$-value. Mediation analyses were conducted only for the outcome measures that were significantly affected by group in the regression analyses on the outcome measures.

The significance level was determined at $\alpha<.05$, and tests were two tailed. The analyses were conducted using SPSS10.

Table 5.1. Evaluation of the tailored and the generic information programmes on general aspects, and on the specific information on fat, fruit and vegetables (mean, standard deviation and F-value)

\begin{tabular}{|c|c|c|c|}
\hline & $\begin{array}{c}T \\
(n-168)\end{array}$ & $\begin{array}{c}\text { GI } \\
(n-176)\end{array}$ & $\begin{array}{c}\text { F-value } \\
(d f=1,341)\end{array}$ \\
\hline General programme aspects & Mean $(S D)$ & Mean (SD) & \\
\hline Attractiveness & $1.08(1.22)$ & $0.89(1.36)$ & 1.89 \\
\hline Ease of use & $1.36(1.41)$ & $1.50(1.35)$ & 0.88 \\
\hline Enjoyability & $1.51(1.15)$ & $1.26(1.38)$ & 3.49 \\
\hline Comprehensibility & $2.05(1.04)$ & $2.06(1.03)$ & 0.01 \\
\hline Interestingness & $1.34(1.31)$ & $0.93(1.44)$ & $7.78^{* *}$ \\
\hline How much information read & $2.01(1.51)$ & $2.13(1.31)$ & 0.55 \\
\hline \multicolumn{4}{|l|}{ Information on fat } \\
\hline Individualisation & $0.82(1.66)$ & $-0.64(1.78)$ & $61.53 \cdots$ \\
\hline Personal relevance & $0.85(1.65)$ & $-0.15(1.82)$ & $28.38 \cdots$ \\
\hline Novelty & $-0.43(1.90)$ & $-1.79(1.48)$ & $55.22 * \cdots$ \\
\hline Credibility & $1.45(1.37)$ & $1.70(1.47)$ & 2.70 \\
\hline Usefulness & $1.31(1.41)$ & $1.23(1.55)$ & 0.26 \\
\hline \multicolumn{4}{|l|}{ Information on vegetables } \\
\hline Individualisation & $0.79(1.66)$ & $-0.48(1.79)$ & $46.42 \cdots$ \\
\hline Personat relevance & $0.88(1.65)$ & $0.07(1.80)$ & $18.49 \cdots$ \\
\hline Novelty & $-0.55(1.82)$ & $-1.81(1.35)$ & $53.41^{\cdots}$ \\
\hline Credibility & $1.38(1.32)$ & $1.81(1.19)$ & $9.85^{*}$ \\
\hline Usefulness & $1.22(1.38)$ & $1.24(1.44)$ & 0.03 \\
\hline \multicolumn{4}{|l|}{ Information on fruit } \\
\hline Individualisation & $0.85(1.68)$ & $-0.40(1.94)$ & $40.29 * \cdots$ \\
\hline Personal relevance & $0.81(1.63)$ & $0.15(1.91)$ & $11.64 *$ \\
\hline Novelty & $-0.78(1.70)$ & $-1.68(1.41)$ & $28.34 \cdots$ \\
\hline Credibility & $1.57(1.25)$ & $1.85(1.13)$ & $4.65^{*}$ \\
\hline Usefulness & $1.29(1.37)$ & $1.40(1.39)$ & 0.55 \\
\hline
\end{tabular}

Note. $\mathrm{TI}$ - Tailored information, $\mathrm{Gl}$ - Generic information. All items measured on scales ranging from -3 to $+3 ; * p<.05, \cdots p<.01, \cdots p<.001$. 


\section{Results}

\section{Participants}

The sample of 616 respondents that was valid for analysis had a mean age of 42 years $(S D=9)$ : $43 \%$ were female and $94 \%$ had been born in the Netherlands. Eleven percent had a university degree, $30 \%$ had completed higher professional training. $23 \%$ had completed high school, $35 \%$ had completed intermediate or lower level professional training and $1 \%$ had completed primary school only.

Participants who dropped out did not differ significantly from those who participated in the study, on any of the characteristics tested or on the outcome measures. Respondents who dropped out were, however, more likely to be in the tailored intervention (odds ratio $[O R]=3.5,95 \%$ confidence interval $[C]=2.1-5.7$ ) and the generic information $(O R=2.9, C I=1.7-4.8)$ groups, than in the no information control group. There was no significant difference in drop out rate between the tailored and generic information groups. Comparison of the group compositions after drop out revealed that the groups did not differ significantly on the characteristics tested or on the outcome measures.

\section{Evaluation and use of the interventions}

The tailored intervention was rated as more enjoyable, interesting. personally relevant, individualised and new than the generic nutrition information (Table 5.1). The generic information on fruit and vegetables was rated as being more credible than the tailored information. The results on evaluation measures were found to be the same for the 'at risk' and 'unaware' subgroups. The tailored intervention programme was used less frequently $(M=1.8$ times, $S D=1.6)$ than the generic information programme $(M=2.6$ times, $S D=3.0) \mid F(1,346)=10.96 . p=.001\}$. Respondents in the tailored intervention group spent more time on using the programme $(M$ $=22$ minutes in each session, $S D=15$ ) than respondents in the generic information group $(M=18$ minutes in each session, $S D=19)[F(1,326)=4.49, p=.035]$.

\section{Awareness, intention to change and intake}

Baseline and post-test mean scores on the outcome measures, as well as standardized regression coefficients ( $\beta$-values) for the group effects at post-test, are shown in Table 5.2. As regards fat and vegetable consumption, group effects were found for self-rated intake and intention to change. indicating a lower level of awareness and a lower intention to change in the control groups than in the tailored intervention group. A group effect for vegetable intake indicated a (modestly) lower post-test intake level in the control groups compared to the tallored intervention group. No significant group effects were found for fruit intake.

The $\beta$-values from the regression analyses for the 'at risk' and 'unaware' subgroups are shown in Table 5.3. These subgroups showed similar. but stronger, 
Table 5.2. Baseline and post-test mean scores on the outcome measures for each study group. Standardized regression coefficients (8-values) from the multiple regression analyses on the outcome measures, with the tailored information group as the reference group

\begin{tabular}{|c|c|c|c|c|c|c|c|c|}
\hline & \multicolumn{3}{|c|}{$\begin{array}{c}\text { Baseline } \\
\text { Mean (SD) }\end{array}$} & \multicolumn{3}{|c|}{$\begin{array}{l}\text { Post-test } \\
\text { Mean (SD) }\end{array}$} & \multicolumn{2}{|c|}{ B-values } \\
\hline & TI & GI & NI & $\mathrm{TI}$ & GI & NI & GI & NI \\
\hline Fat & $n=182$ & $n=184$ & $n=222$ & $n=182$ & $n-184$ & $n=222$ & & \\
\hline Self-rated intake" & $-0.25(0.68)$ & $-0.16(0.70)$ & $-0.11(0.81)$ & $-0.12(0.76)$ & $=0.22(0.71)$ & $-0.18(0.73)$ & $-10^{\circ}$ & $-10 * 0$ \\
\hline Intention to change & $-0.23(0.92)$ & $-0.13(0.95)$ & $-0.21(1.01)$ & $0.01(0.97)$ & $-0.13(0.99)$ & $-0.24(1.00)$ & $-.09^{*}$ & $-12 \%$ \\
\hline Fat intake & $19.8(6.1)$ & $20.0(5.9)$ & $20.3(6.2)$ & $19.2(6.2)$ & $19.2(6.0)$ & $19.9(6.2)$ & -.02 & .02 \\
\hline Vegetables & $n=133$ & $n-142$ & $n=166$ & $n=133$ & $n=142$ & $n=166$ & & \\
\hline Self-rated intake" & $0.35(0.67)$ & $0.44(0.68)$ & $0.42(0.71)$ & $0.16(0.69)$ & $0.37(0.71)$ & $0.37(0.70)$ & $.14 * 0$ & $.13^{*}$ \\
\hline Intention to change" & $-0.38(0.78)$ & $-0.32(0.83)$ & $-0.31(0.85)$ & $-0.04(0.93)$ & $-0.25(0.81)$ & $-0.26(0.89)$ & $-13^{\circ}$ & $-14 * 0$ \\
\hline Vegetable intake & $1.8(0.8)$ & $1.8(0.8)$ & $1.9(1.0)$ & $1.9(0.9)$ & $1.7(0.8)$ & $1.8(0.9)$ & $-.08^{\circ}$ & $.07^{*}$ \\
\hline Fruit & $n=135$ & $n=140$ & $n=167$ & $n=135$ & $n=140$ & $n=167$ & & \\
\hline Self-rated intake & $-0.19(1.09)$ & $-0.26(0.95)$ & $-0.22(0.99)$ & $-0.19(1.05)$ & $-0.16(0.89)$ & $-0.26(0.91)$ & .06 & -.02 \\
\hline Intention to changed & $-0.10(0.88)$ & $0.02(0.90)$ & $-0.08(0.93)$ & $-0.01(1.01)$ & $-0.04(0.92)$ & $-0.13(0.85)$ & -.05 & .07 \\
\hline Fruit intake & $2.2(1.6)$ & $2.1(1.4)$ & $2.1(1.4)$ & $2.3(1.6)$ & $2.0(1.2)$ & $2.0(1.6)$ & -.06 & -.04 \\
\hline
\end{tabular}

Note. $\mathrm{Tl}$ - Tailored information, GI - General information, $\mathrm{NI}=$ No information. "Measured on scales ranging from -2 to +2 . Fat intake is expressed in fat points, vegetable and fruit intake in servings per day. ${ }^{\prime} p<.10,{ }^{*} p<.05,{ }^{*}{ }_{p}<.01$. 
Table 5.3. Standardised regression coefficients (B-values) for group effects in the multiple regression analyses on the outcome measures in the 'at risk' and 'unaware' subgroups. The tailored information group is the reference group

\begin{tabular}{|c|c|c|c|c|}
\hline \multirow{2}{*}{ 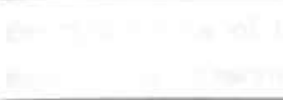 } & \multicolumn{2}{|c|}{ Gl } & \multicolumn{2}{|c|}{$\mathrm{NI}$} \\
\hline & At risk & Unaware $^{b}$ & At risk & Unaware \\
\hline Fat & $n=316$ & $n=271$ & $n-316$ & $n=271$ \\
\hline Self-rated intake & $-.16 \%$ & $-16 \%$ & $-.18 * 0$ & $-.14^{*}$ \\
\hline Intention to change & $-.14^{*}$ & $-14^{*}$ & $-.22 \cdots$ & $-.24 \cdots$ \\
\hline Fat intake & .02 & -.02 & .02 & .01 \\
\hline Vegetables & $n=361$ & $n=348$ & $n-361$ & $n=348$ \\
\hline Self-rated intake & $.14 * *$ & $.14^{*}$ & $.15 *$ & $.17 *$ \\
\hline Intention to change & $-.16 \%$ & $-.15^{\circ}$ & $-.20 \cdots$ & $-.20 *$ \\
\hline Vegetables intake & $-.11^{\circ}$ & -.07 & -.07 & -.06 \\
\hline Fruit & $n=226$ & $n-102$ & $n=226$ & $n-102$ \\
\hline Self-rated intake & .07 & .03 & -.01 & -.09 \\
\hline Intention to change & -.06 & $-.22^{*}$ & $-.16^{\circ}$ & $-.32 * \bullet$ \\
\hline Fruit intake & -.01 & -.20 & $-.15^{\circ}$ & $-.27^{*}$ \\
\hline
\end{tabular}

Note. GI - general nutrition information control group, $\mathrm{NI}$ - no information control group. "Subgroup of respondents with intake levels not in agreement with recommendations, bSubgroup of respondents who underestimate their fat intake and overestimate their fruit and vegetable intake, CNumbers of respondents (n) refer to all respondents in the analyses. ${ }^{*} p<.05,{ }^{*} p<.01,{ }^{*}{ }^{*} p<.001$.

group effects for the determinants of fat and vegetable intakes compared to the total study population. As regards fruit consumption, group effects were found for intention to change and fruit intake; respondents in both control groups had a lower intention to change and respondents in the no information control group had a lower fruit intake than those in the tailored intervention group.

Repeating the analyses in an intention-to-treat procedure, resulted in smaller $\beta$-values and slightly higher but still significant $p$-values. An exception are the effects on vegetable and fruit intake in that the few group differences that were found remained only borderline significant.

\section{Mediator analyses}

Correlations of the variables included in the mediator analyses are displayed in Table 5.4. Significant mediation was found for perceived personal relevance and individualisation on self-rated fat intake and for perceived personal relevance, individualisation and interestingness on intention to change fat intake. Perceived personal relevance and individualisation significantly mediated the effects for intention to change vegetable intake (Table 5.5). In the mediator analysis the group effect for vegetable intake was not significant $(\beta=.08, p=.09)$ and therefore mediator analysis could not be conducted for vegetable intake. Group did not significantly affect interestingness in the analysis on self-rated vegetable intake and therefore mediator analysis could not be conducted. 
Table 5.4. Pearson correlation coefficients of the variables in the mediator analyses

\begin{tabular}{|c|c|c|c|c|c|c|}
\hline & 1 & 2 & 3 & 4 & 5 & 6 \\
\hline \multicolumn{7}{|l|}{ 1. Fat intake } \\
\hline 2. Intention to reduce fat intake & $\begin{array}{c}.03 \\
n-588\end{array}$ & & & & & \\
\hline 3. Self-rated fat intake & $\begin{array}{c}29 * \cdots \\
n-588\end{array}$ & $\begin{array}{c}27 \cdots \\
n-588\end{array}$ & & & & \\
\hline 4. Perceived interestingness & $\begin{array}{l}-07 \\
n-328\end{array}$ & $\begin{array}{c}23 \cdots \\
n-328\end{array}$ & $\begin{array}{l}-.05 \\
n-328\end{array}$ & & & \\
\hline 5. Perceived personal relevance & $\begin{array}{c}.04 \\
n-328\end{array}$ & $\begin{array}{c}46 \cdots \\
n-328\end{array}$ & $\begin{array}{c}24 \cdots \\
n-328\end{array}$ & $\begin{array}{c}38 * \cdots \\
n-328\end{array}$ & & \\
\hline 6. Perceived individualisation & $\begin{array}{l}02 \\
n-329\end{array}$ & $\begin{array}{c}30 * \\
n=329\end{array}$ & $\begin{array}{c}22 \cdots \\
n-329\end{array}$ & $\begin{array}{c}33 \cdots \\
n-329\end{array}$ & $\begin{array}{c}69 * \cdots \\
n=329\end{array}$ & \\
\hline & 1 & 2 & 3 & 4 & 5 & 6 \\
\hline \multicolumn{7}{|l|}{ 1. Vegetable intake } \\
\hline 2. Intention to increase vegetable intake & $\begin{array}{l}-11^{*} \\
n-440\end{array}$ & & & & & \\
\hline 3. Self-rated vegetable intake & $\begin{array}{l}.44 \cdots \\
n=441\end{array}$ & $\begin{array}{l}-28+\cdots \\
n-440\end{array}$ & & & & \\
\hline 4. Perceived interestingness & $\begin{array}{c}.02 \\
n-250\end{array}$ & $\begin{array}{c}.24 \cdots \\
n-250\end{array}$ & $\begin{array}{l}-.03 \\
n-250\end{array}$ & & & \\
\hline 5. Perceived personal relevance & $\begin{array}{l}-.15^{*} \\
n=251\end{array}$ & $\begin{array}{c}.34 * \cdots \\
n-251\end{array}$ & $\begin{array}{l}-.23 \cdots \\
n-251\end{array}$ & $\begin{array}{l}42 \cdots \\
n-250\end{array}$ & & \\
\hline 6. Perceived individualisation. & $\begin{array}{l}-.06 \\
n=251\end{array}$ & $\begin{array}{l}30 \cdots \\
n=251\end{array}$ & $\begin{array}{l}-19 * \\
n=251\end{array}$ & $\begin{array}{l}.35 \cdots \\
n=250\end{array}$ & $\begin{array}{c}68 \cdots \\
n-251\end{array}$ & \\
\hline
\end{tabular}

Note. ${ }^{*} p<.05, \cdots p<.01, \cdots p_{i}<.001$.

Table 5.5. Standardised regression coefficient (B) and p-value for the treatment group variable (tailored vs. generic), uncontrolled and controlled for potential mediator variables

\begin{tabular}{|c|c|c|c|c|c|c|c|c|}
\hline & \multicolumn{2}{|c|}{$\begin{array}{l}\text { Uncontrolled } \\
\text { treatment group } \\
\text { effect }\end{array}$} & \multicolumn{2}{|c|}{$\begin{array}{c}\text { Controlled for } \\
\text { personal } \\
\text { relevance }\end{array}$} & \multicolumn{2}{|c|}{$\begin{array}{l}\text { Controlled for } \\
\text { individualisation }\end{array}$} & \multicolumn{2}{|c|}{$\begin{array}{l}\text { Controlled for } \\
\text { interestingness }\end{array}$} \\
\hline & B & $p$-value & B & $p$-value & B & $p$-value & B & p-value \\
\hline \multicolumn{9}{|l|}{ Fat } \\
\hline Self-rated intake & .09 & .04 & $.06^{\mathrm{a}}$ & .17 & $.03^{\mathrm{b}}$ & .49 & .09 & .05 \\
\hline Intention to change & .10 & .04 & $.00^{\mathrm{b}}$ & .95 & $.00^{b}$ & .94 & $.08^{a}$ & .13 \\
\hline \multicolumn{9}{|l|}{ Vegetables } \\
\hline Self-rated intake & -.15 & .00 & -.14 & .01 & -.14 & .01 & - & \\
\hline Intention to change & .14 & .02 & $.07^{b}$ & .22 & $.04^{b}$ & .49 & .11 & .05 \\
\hline Vegetable intake & .08 & .09 & - & & - & & - & \\
\hline
\end{tabular}

Note. aSignificant mediation p-values for $z$-scores $<.05$ in the analyses testing significance of the mediation effects, bSignificant mediation p-values for $z$-scores $<.01$. 


\section{Discussion}

The web-based tailored nutrition information resulted in a significant effect on the determinants of fat, fruit and vegetable intake, whereas effects on intakes were modest or absent. Participants evaluated the taflored information more favourably than the generic information. The results partly support our hypothesis that perceived personal relevance, individualisation and interestingness mediated the effects of the tailored intervention.

The results of the present study are largely consistent with the findings of other studies examining the effects of interactive and multi-media applications of computer-tailored nutrition interventions. Previous studies found tailoring effects on determinants of fat, fruit, vegetables and fibre consumption (Anderson et al., 2001: Campbell et al., 1999; Oenema et al., 2001), and on intakes of these nutrients and foods (Anderson et al., 2001). The effects on awareness and intention to change in the present study are promising. since these factors are prerequisites for moving to further stages of behaviour change (Weinstein. 1988). However, a tailoring effect on actual behaviour was also expected. In the present study, tailoring effects on behaviour were restricted to vegetables intake, and fruit intake only in the 'at risk' and 'unaware' subgroups. No effects on fat intake were found, whereas in prior studies evaluating the effects of printed tailored nutrition interventions the effects on fat intake have been found most consistently (Brug et al., 1999; De Bourdeaudhuij \& Brug, 2000).

The tailored information provided in the present study was derived from previously tested printed tailored information that had produced effects on fat intake. Therefore, it is unlikely that the lack of effect on fat intake was caused by insufficient behavioural change information. The difference in impact on fat intake between the web-based and printed tailored information may, however, be an indication that tailored information provided through an interactive channel is not as effective in influencing behaviour as printed tailored information.

Several advantages have been ascribed to the Internet as a channel for providing health information, including its greater flexibility, its interactive nature which requires active involvement of the recipient, the possibility of incorporating aspects of interpersonal communication in the information provided, the potential to reach large audiences, the immediate presentation of taflored information which may enhance the accuracy of the tailored information, and a greater openness in communication (Fotheringham, Owies, Leslie, \& Owen, 2000: Oenema et al., 2001; Stout, Villegas, \& Kim, 2001: Strecher et al. 1999). Printed media may, however. have advantages over the Internet as a medium for providing health information aimed at achieving behavioural changes. It may, for example, be easier to read printed information more thoroughly than information on the screen. and it may' be easier to reread printed information that is sent by mail than having to make the effort of starting up a programme on the computer or the Internet first. Furthermore, printed information is delivered in a predetermined form, whereas the appearance and delivery of web-based information may vary, depending on the 
settings and capacity of the computer used by the recipient. To our knowledge, no previous studies have tested the difference in effects on behaviour of taflored information provided through the Internet or through print media, but there is a need for research to examine this.

Another issue that may have caused the lack of an effect on fat intake is the time span between using the programme and filling out the post-test questionnaire. Only if respondents used the programme right after they had received it, would there have been sufficient time for behaviour change. Some respondents may, however, not have followed the instructions and used the programme later.

Finally, the one study to date that did find an effect of interactive tailored information on fat intake used multiple, weekly segments (Anderson et al., 2001). In the present study it was possible for respondents to use the programme as often as they liked. The frequency of use of the tailored intervention programme was on average 1.8 times, which may not have been frequent enough to result in improvement in behaviour that significantly differed from behaviour in the control groups. The finding that the generic information programme was used more frequent than the tailored intervention may indicate that it was easier and less time consuming to use this shorter programme.

Perceived personal relevance, perceived individualisation, novelty of information and interactivity have been identified as important features of effective nutrition education (Contento et al., 1995). Like previously tested tailored information (Brug et al. 1999: De Bourdeaudhuif \& Brug. 2000: Oenema et al. 2001), the present intervention does seem to incorporate these features. The Elaboration Likelihood. Model (Petty \& Cacioppo, 1986) posits that individualisation and personal relevance of the information may motivate to process information via the so-called central route, i.e., effortful and thoughtfully, resulting in relatively strong and sustainable attitudes that are more predictive for intention and behaviour. The present study found some evidence that these features do indeed mediate the effects of the tailored information. Tailoring effects on determinants of fat and vegetable intake were mediated by perceived personal relevance and perceived individualisation. The finding that not all talloring effects were mediated by the features studied suggests that computer tailoring involves more than just increasing perceived personal relevance and perceived individualisation. The fact that tailored information can be tailored to an individuals' motivational stage of change, which may cause less resistance to the message and make stage transitions more likely, has been suggested as another mechanism through which tailored information may exert its effects (Dijkstra \& De Vries, 1999: Strecher, Kreuter, Den Boer, Kobrin, Hospers, \& Skinner, 1994: Weinstein \& Sandman, 1992).

The finding that the tailored information was rated as less credible than the generic information has been reported before. Recipients of negative or unexpected feedback have been found to respond by doubting the accuracy and credibility of the feedback information (Bowen et al., 1994: Van Assema et al. 1996). In the present study, however, credibility ratings for tailored and generic information were both positive, though not to the same degree. Additional analyses revealed 
that, in line with the prior findings, respondents with a lower than recommended fruit intake were responsible for the lower credibility ratings of the tailored information. As regards the tailored information about vegetable consumption, all respondents, but particularly those with diets consistent with recommendations and aware of their intake, contributed to the lower credibility ratings. The programme's assessment of vegetable intake is brief, and feedback is based on this brief assessment. Respondents who consume sufficient amounts of vegetables may have felt that this was not accurate enough.

There are a number of limitations to the study that need to be mentioned. Since the present study comprised a self-selected study population, it may have included a relatively large proportion of people who were specifically interested in nutrition. Furthermore, respondents who were not interested in using a computer to obtain information about nutrition may have refused to participate, and potential participants who did not have access to a computer with a CD-ROM drive could not be included in the study. The drop out rates in the tailored information group and the generic information control group were larger than that in the no information control group. This may have been due to the effort required to use the information programme. Respondents who did not use the programme because of lack of time or technical problems may not have returned the questionnaire. Since the subjects who did not drop out did not differ on the outcome measures or other important characteristics at baseline, there is no proof that the drop out biased the results. Even though self-reported information on use of the tallored and generic information programmes was obtained, the study lacks objectively monitored information on how often respondents visited the programme, how long they stayed on pages and how many pages they went through. Finally, since this study only examined the short-term efficacy of the tailored information in a controlled situation, it cannot provide information about effectiveness in real life situations or in the long term.

The present study provides some evidence that a web-based computer-tallored nutrition intervention can have a short-term effect on awareness and intention to change fat, fruit and vegetable intake. The effects of the tailored information may be partly explained by the greater perceived personal relevance and individualisation of the information. Future studies should examine longer-term effects and compare web-based tailored information with tailored information provided through printed media. 


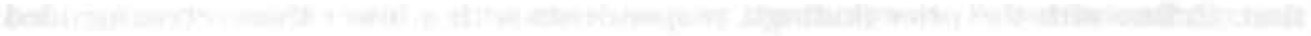

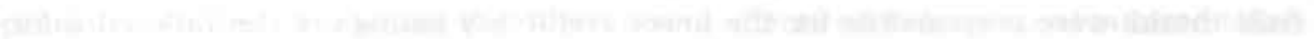

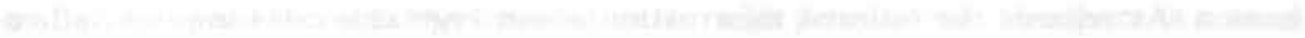
7.

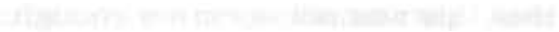




\title{
Long-term efficacy of a web- based computer-tailored nutrition intervention
}

\begin{abstract}
A web-based computer-tailored nutrition intervention was evaluated for the longerterm (6 months) effects on fat, fruit and vegetable intake and its determinants. In a randomised trial, the computer-tailored intervention was compared with web-based generic nutrition information and no information. Respondents with baseline intake levels not in agreement with recommendations were included in the analyses applying an intention-to-treat procedure. At post-test respondents in the tailored intervention group were more aware of their vegetable intake and had a greater intention to change their vegetable and fat intake than respondents in the control groups. Respondents in the tailored intervention group consumed significantly more vegetables than those in the no information control group. The tailored information induced cognitive processing. but impact on consumption levels was very limited.
\end{abstract}

Submitted as short report as: Oenema, A., \& Brug, J. Long-term efficacy of a web-based computertailored nutrition intervention. 


\section{Introduction}

Diets low in saturated fat and high in fruit and vegetables contribute to the preven. tion of chronic diseases (Rolls \& Shide, 1992; Willet, 1994). The majority of populations in Western countries have a higher saturated fat intake and a lower fruit and vegetable intake than recommended (Beer-Borst et al, 2000: Ganji \& Betts, 1995). There is thus a need for effective healthy diet promotion interventions that can reach many people.

Computer-tailored printed information has repeatedly been found effective in inducing dietary changes, especially in reducing fat intake (Brug et al., 1999). Interactive and multi-media applications of computer-tailored nutrition information are now feasible. Providing tallored nutrition information interactively via the Internet would yield many advantages for health education practice, and the channel is regarded as a promising one by researchers. Since the Internet has become a widely used source of health information, it has the potential to reach many people. Disseminating tailored interventions through the Internet would require a minimum of human effort and resources, making it possible to reach large groups of people at low costs. Furthermore, the process of providing tailored information could be made truly interactive, and tailored information would become readily avallable, as long as there is a computer online. Before providing an interactive computer-tailored intervention through the Internet, however, it is important to explore its efficacy.

Computer-based, multimedia and web-based applications of computer-tailored nutrition interventions have been found to be efficacious in modifying determinants of saturated fat, fruit, vegetable and fibre consumption in the short term (Anderson et al., 2001; Campbell et al., 1999; Oenema \& Brug. 2003) and in modifying intakes of saturated fat, fruit and vegetables in the short- and longer-term (Anderson et al., 2001).

The present paper reports on the longer-term effects of a web-based computertallored nutrition information programme on awareness, intention to change and intake of saturated fat, fruit and vegetables.

\section{Methods}

\section{Participants}

A total of 815 respondents (2133 invited) volunteered to participate in the study of which 782 were eligible to be included in the study. The eligible respondents were evenly distributed over the study groups. The response rate at post-test was $73 \%$ in the tailored intervention group. $76 \%$ in the generic information group and $83 \%$ in the no information control group. 


\section{Study design and procedure}

The computer-tailored intervention was compared with web-based generic nutrition information and no information in a randomised trial with a pre-test and a six month post-test.

Participants were recruited by sending a letter to employees at seven companies. and government and health organisations, explaining the purpose and procedure of the study. The employees were invited to enroll in the study by completing the informed consent form and the baseline questionnaire that were sent along with the invitation letter. Respondents who did not have access to a computer were excluded from the study. Four weeks after the baseline questionnaire had been completed, respondents received the assigned intervention programme, either on. a CD-ROM or through the worksite intranet. Respondents in the no information group received a letter explaining that they would be sent an information programme after returning the post-test questionnaire. Six months post intervention, questionnaires were mailed to the participants and initial non-responders were sent a reminder letter once. Participants could win one of twenty $€ 20$ gift vouchers. as an incentive to participate.

\section{Interventions}

The information programmes consisted of HTML pages interlinked to form a website. The tailored information programme provided personal and normative feedback on intake levels of fat, fruit and vegetables, and individualised suggestions on how to decrease saturated fat, or increase fruit and vegetable intake. The tailored information programme has been described in more detall elsewhere (Oenema \& Brug, 2003; Oenema, Tan, \& Brug, accepted). The generic information programme discussed the health benefits of diets low in fat and high in fruit and vegetables. and stressed that many people are not aware of their dietary intake levels. General suggestions were given for decreasing saturated fat and increasing fruit and vegetable intake. The generic information was also provided in a web-based form. to make the programmes appear as similar as possible. Both information programmes included an extensive list of low saturated fat and high vegetable recipe suggestions that could be browsed.

\section{Measures}

Fat, fruit and vegetable intakes were measured by means of validated food frequency questionnaires (Bogers et al., accepted; Van Assema et al, 2001: Van Assema et al., 2002). The questionnaire for fat assessed the intake of foods and food products that contribute most to saturated fat intake in the Dutch diet. This questionnaire was used to calculate a fat score, ranging from 0 to 80 , reflecting total saturated fat intake per day. The fruit and vegetable questionnaire included questions on intake of raw and cooked vegetables and fruit and fruit juice, allowing the average intake 
in servings of fruit and vegetables per day to be computed. Self-rated intake and intention to change were measured for all three dietary behaviours using single items on five-point bipolar scales ['how much fat/fruit/vegetables do you think you eat?' (very little - very much) and 'Do you intend to start eating less fat/more fruit/vegetables than you do now?" (certainly do not - certainly do)]. Self-rated intake adjusted for objectively assessed intake was used as an indicator of awareness (Brug et al., 1998b). Intakes and determinants were assessed at baseline and posttest. Gender, age, education, ethnic background, height, weight, and household composition were queried at baseline.

\section{Statistical analyses}

Binary logistic regression analyses were conducted to examine predictors of drop out, using drop out as the dependent variable and study group. gender, age, education, body mass index, and baseline measures of self-rated intake, intention to change, and assessed intake as the independent variables. These analyses were also conducted with the study group as the dependent variable, to examine the success of the randomisation.

Multuple linear regression analyses were conducted to test for group differences at post-test. Group was dummy coded with the tailored intervention group as the reference group. The post-test score of the outcome indicators (self-rated intake, intention to change and assessed intake) were the dependent variables, and the dummies for group and the baseline measure of the outcome indicator were the Independent variables. The analyses on self-rated intake included food-frequency scores at post-test as an additional independent variable, to create the indicator of awareness. An intention-to-treat procedure was applied, and only respondents with a 'need to change', i.e., those with baseline intake levels inconsistent with recommendations (the at risk group), were included in the analyses. The analyses were two-tailed and an $\alpha$-level of $p<.05$ was regarded as significant and of $p<.10$ as borderline significant. All analyses were conducted using SPSS10.

\section{Results}

\section{Participants}

The mean age of the respondents was $42 .(S D=9) .41 \%$ were female and $95 \%$ had been born in the Netherlands. Forty-one percent had a university degree or had completed higher professional training. 23\% had completed high school, $34 \%$ had completed a lower or intermediate professional training and $1 \%$ had completed primary school only. Fifty-two percent of the respondents were in the 'at risk' group for fat consumption, $85 \%$ for vegetable consumption and $52 \%$ for fruit consumption.

Participants who dropped out were more likely to be in the tailored (odds ratio 
Table 6.1. Baseline and post-test mean scores (SD) for the outcome measures. Standardised regression coefficients (B) from the multiple linear regression analyses with the tailored intervention group as the reference group

\begin{tabular}{|c|c|c|c|c|c|c|c|c|}
\hline & \multicolumn{3}{|c|}{ Baseline } & \multicolumn{3}{|c|}{ Post-test } & \multicolumn{2}{|c|}{ B } \\
\hline & $\mathrm{TI}$ & GI & NI & $\mathrm{TI}$ & GI & NI & GI & $\mathrm{NI}$ \\
\hline Fat consumption & $n=133$ & $n-128$ & $n=135$ & $n=133$ & $n=128$ & $n=135$ & & \\
\hline Self-rated intake & $=0.07(0.61)$ & $-0.06(0.65)$ & $-0.05(0.67)$ & $-0.09(0.66)$ & $-0.11(0.61)$ & $-0.02(0.61)$ & -.03 & -.01 \\
\hline Intention to change & $-0.20(0.88)$ & $-0.13(1.01)$ & $-0.21(0.93)$ & $-0.08(1.00)$ & $-0.25(1.06)$ & $-0.30(0.93)$ & $-.10^{*}$ & $-11^{*}$ \\
\hline Intake & $24.0(4.1)$ & $24.3(4.2)$ & $24.8(4.7)$ & $22.1(4.9)$ & $23.0(4.8)$ & $22.8(4.9)$ & .06 & 01 \\
\hline Vegetable consumption & $n=167$ & $n=183$ & $n=163$ & $n=167$ & $n-183$ & $n=163$ & & \\
\hline Self-rated intake & $0.25(0.62)$ & $0.36(0.66)$ & $0.33(0.65)$ & $0.17(0.61)$ & $0.32(0.65)$ & $0.33(0.67)$ & $.06^{\circ}$ & $10^{\circ}$ \\
\hline Intention to change & $-0.25(0.79)$ & $-0.37(0.83)$ & $-0.32(0.84)$ & $-0.05(0.86)$ & $-0.33(0.82)$ & $-0.35(0.84)$ & $-12 *$ & $.14 *$ \\
\hline Intake & $1.5(0.4)$ & $1.5(0.5)$ & $1.5(0.5)$ & $1.6(0.6)$ & $1.6(0.7)$ & $1.4(0.6)$ & .01 & $+10^{\circ}$ \\
\hline Fruit consumption & $n=103$ & $n=115$ & $n=101$ & $n-103$ & $n-115$ & $n=101$ & & \\
\hline Self-rated intake & $-0.80(0.83)$ & $-0.70(0.88)$ & $-0.64(0.92)$ & $-0.69(0.90)$ & $-0.63(0.83)$ & $-0.54(0.82)$ & .00 & .03 \\
\hline Intention to change & $0.14(0.91)$ & $0.13(0.89)$ & $0,17(0.91)$ & $0.03(1.00)$ & $0.11(0.85)$ & $0.16(0.91)$ & .05 & .06 \\
\hline Intake & $1.1(0.5)$ & $1.1(0.6)$ & $1.0(0.5)$ & $1.3(0.8)$ & $1.3(0.7)$ & $1.3(0.9)$ & -.05 & -.02 \\
\hline
\end{tabular}

Note. $\mathrm{N}$ - tailored information group, GI - general information group, $\mathrm{NI}=$ no information group. Scales for self-rated intake and intention to change ranged from -2 to +2 . Fat intake is expressed in fat points, vegetable and fruit intake in servings per day. ${ }^{\prime} p<.10,{ }^{*} p<.05,{ }^{*} p<.01$. 
$[O R]=1.91,95 \%$ confidence interval $[C I]=1.23-2.97)$ or generic $(O R=1.66, C I=$ $1.07-2.60$ ) information groups than in the no information group, and were more likely to be younger $(O R=0.97, C I=0.95-0.99)$. There were no significant baseline differences between the groups.

\section{Awareness, intention to change and intake}

At six months post-intervention, respondents in the generic information and no information groups had significantly lower mean scores for intention to change fat intake, compared to those in the tailored intervention group (Table 6.1). Respondents in the generic information and no information groups rated their vegetable intake higher, indicating a lower level of awareness, and had a significantly lower mean score for the intention to change compared to those in the tailored intervention group. Respondents in the no information control group had a significantly lower vegetable intake than those in the tailored intervention group. No significant group differences were found for fruit consumption.

\section{Discussion}

This study revealed effects of a web-based computer-tailored nutrition education programme on the intention to change fat intake. on awareness of and intention to change vegetable intake, and on the actual intake of vegetables among respondents with risk behaviour at baseline, six months after the intervention had been distributed.

The results of the present study are in line with the findings of a previous shortterm evaluation of the web-based tallored intervention, in that the effects were found primarily on the pre-behavioural determinants (Oenema et al., accepted). This indicates that the tailored information induced cognitive processing, but did generally not induce changes in behaviour that were greater than those in the control groups.

The results are modest compared to those of studies on the effects of printed tailored nutrition interventions. Printed tailored interventions have been found to induce changes in determinants and intake of fruit, vegetables and especially of fat, that lasted over time (Brug et al., 1999). The present intervention was an adaptation of an effective print-delivered tallored nutrition information system. Since the content of the present intervention was very similar to that of the print version. the modest effects on behaviour of this intervention may be associated with the channel through which the information was delivered. Even though providing tailored information interactively through the Internet may have advantages, it may also present barriers to behaviour change. Whereas providing tailored information via the Internet reduces the effort on the provider's side, it may require an increased effort from the recipient. A recipient has to start a computer, go online and find the site or use a CD-ROM, find out how to use the programme, make choices about 
the sequence used to go through the programme, read the information from the screen, decide whether or not to print it, resist the temptation of surfing to other sites and deal with possible technical problems. This requires a certain amount of motivation and effort that may prevent adequate processing of the tailored information. Furthermore, reading information from a computer screen may not be as easy and possibly not as thoroughly as reading print information. A tailored letter sent by postal mail may be read more thoroughly and repeatedly. since it may be easier and more convenient to read and re-read printed information.

The frequency with which the programme was used may have influenced its effects. since a study testing a computer-based tailored intervention that used multiple weekly information segments (Anderson et al., 2001) produced stronger effects than the intervention tested in the present study. In our study, the median use of the programme was once and this may not have been. frequent enough to result in behaviour changes.

The findings of the present study indicate that tailored information provided interactively does not necessarily produce the same effects on behaviour as information provided through more traditional print media. More research is needed into the effects of computer-tailored nutrition interventions provided through the Internet or other interactive channels, preferably compared with printed information.

The study population was self-selected and we used self-administered questionnaires to obtain the information. Even though such an approach is, at present. usual practice for nutrition intervention evaluation studies aimed at a healthy population, it may limit the validity and generalisability of the findings. We applied an intention-to-treat procedure in order to reduce the bias that could have been introduced by the selective drop out, but bias may have remained. 


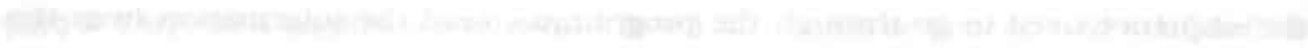

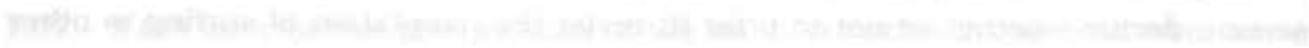

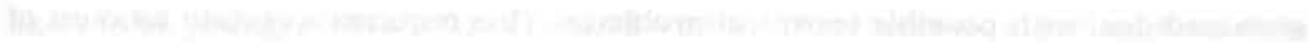

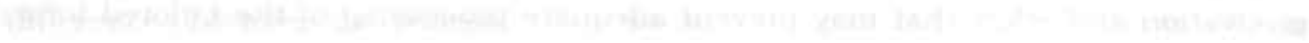




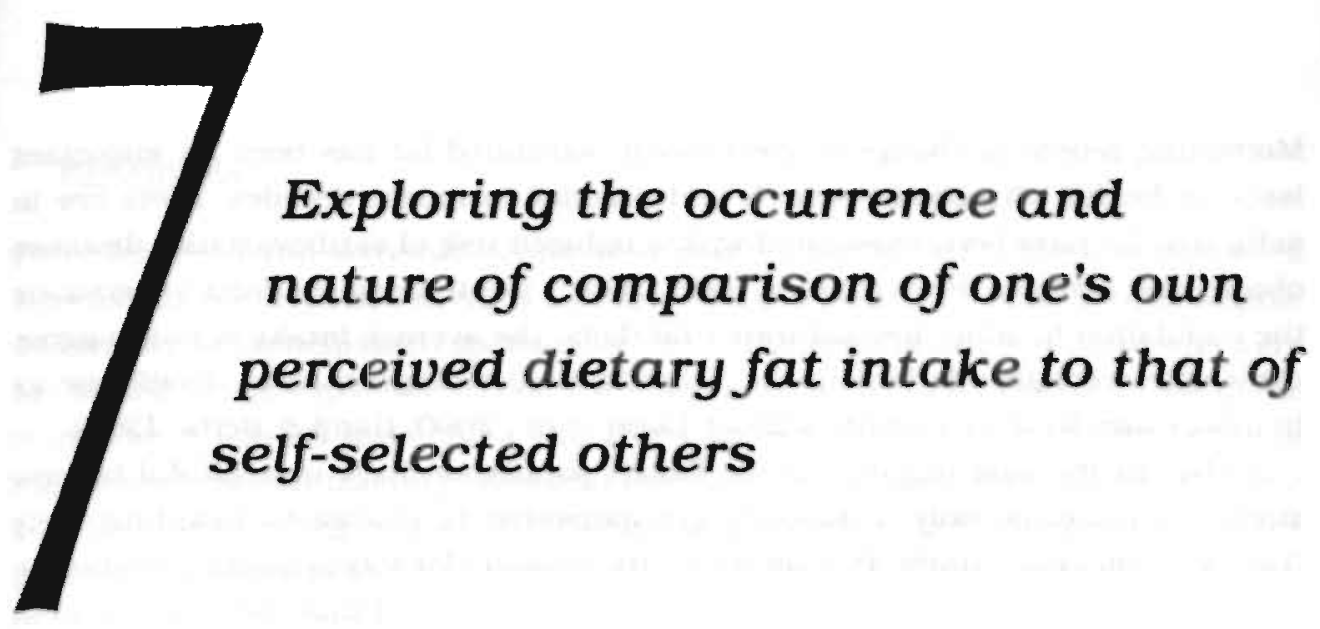

\begin{abstract}
Lack of awareness of personal dietary fat intake is associated with a low motivation to change to a diet lower in fat. An optimistic bias in the comparison of one's own fat intake to that of others is associated with this lack of awareness. Insight into the way people make interpersonal comparisons related to fat intake may contribute to a better understanding of dietary intake and its determinants. Such insight may provide suggestions for nutrition education interventions aimed at increasing awareness and intention to change. The present study explores interpersonal comparisons involved in evaluating personal dietary fat intake. One hundred and eighty-nine respondents participated in structured telephone interviews. The respondents reported that they use interpersonal comparison information related to fat intake to some extent and that they mostly compare themselves with close relatives and friends. Respondents who intended to change to a lower fat diet and younger respondents were more likely to compare themselves with others. Comparison targets were perceived to eat small, as well as large or average amounts of fat, which may indicate that people use positive as well as negative role models for making interpersonal comparisons in terms of dietary fat intake.
\end{abstract}

Published as: Oenema, A., \& Brug, J. (2003). Exploring the occurrence and nature of comparison of one's own perceived dietary fat intake to that of self-selected others. Appetite, 41, 259-264. 


\section{Introduction}

Motivating people to change to diets low in saturated fat has been an important issue in health education research and practice in recent decades. Diets low in saturated fat have been associated with a reduced risk of cardiovascular diseases, obesity and diabetes (Rolls \& Shide, 1992: Willet, 1994). Despite efforts to persuade the population to adopt low-saturated-fat diets, the average intake is not in agreement with recommendations in the Netherlands (Voedingscentrum, 1998) just as in most other Western countries (Beer-Borst et al., 2000; Ganji \& Betts, 1995).

Although the vast majority of the Dutch population have unhealthful fat-consumption patterns, only a minority are motivated to change to healthful diets (Van Wechem et al. 1998). Research into the reasons for this lack of motivation to change has revealed that many Dutch adults are convinced that their diets are not too high in fat and therefore they do not see any need to change (Brug et al. "1998b; Brug et al., 1994).

In line with the Precaution Adoption Process Model (Weinstein, 1988; Weinstein \& Sandman, 1992), that emphasises awareness of personal risk (behaviour) as a prerequisite for moving to further stages of change, this lack of awareness has been identified as a major barrier to the motivation to change complex dietary behaviours, such as saturated fat intake (Brug et al., 1994: Glanz et al. 1997). An optimistic bias in the comparison of ones own risk behaviour to that of others, i.e., believing that one engages in less risky behaviour than others do (cf. Weinstein \& Klein, 1995), seems to be a determinant for lack of awareness of personal risk behaviour, including a high dietary fat intake (Brug et al. 1998b: Weinstein, 1988).

Littie is known, however, about the occurrence and nature of the interpersonal comparisons that are made regarding dietary fat intake. Insight into these comparisons may (1) contribute to a better understanding of dietary behaviour and its determinanis and (2) provide suggestions for designing nutrition education interventions aimed at improving awareness, motivation to change and actual behaviour change. The aim of the present exploratory study is to obtain a basic understanding of interpersonal comparisons in relation to dietary fat intake.

The specific research questions were:

- Do people use interpersonal comparison information to evaluate their fat intake?

- To whom do people compare their fat intake?

- What diet-related comparison information do people use?

- What factors are associated with a tendency to engage in interpersonal comparison of dietary fat intake?

- Do people compare themselves mostly to others whom they perceive to consume small, large or average amounts of fat? 


\section{Methods}

\section{Participants}

Three-hundred and sixty-two people were reached at the 390 addresses that were selected for participation in the study and 189 (64 men, 125 women) were eligible and willing to participate (response rate 52\%). The main reasons of those who were not willing to participate were lack of interest and lack of time. The mean age of the respondents was 43 years $(S D=12)$. Thirty-six percent had a university degree or higher professional training, $42 \%$ had intermediate or lower professional training. $20 \%$ had finished high school and $2 \%$ had primary school only. Sixty percent of the participants had a paid job outside the home, $19 \%$ reported housekeeping as their main occupation, $10 \%$ were unemployed, $7 \%$ were retired and $4 \%$ were students. Most of the respondents lived with one or more others (84\%). The mean body mass index (BMI) of the respondents was $24.3(S D=3.6) \mathrm{kg} / \mathrm{m}^{2}$.

\section{Procedure}

The study was conducted in the spring of 2000. Data were collected by means of structured telephone interviews, which were conducted by trained telephone interviewers. Participants were recruited from a random sample of 390 addresses from the databank of home telephone numbers of the Dutch Telecom Company. In a letter that was sent to the residents at these addresses, it was explained that they had been selected for a study about food habits and that they would be phoned and asked to participate. The letter also explained that participation would mean answering questions, which would take about 15 minutes, and that people between the ages of 18 and 65 could participate. The interviewers called the selected addresses and asked whether there was someone in this age bracket who was at home and was willing to participate in the study. Those who volunteered to participate were either interviewed right away or were called again at a more convenient time. Addresses where the phone was not answered were called again, up to three times. Participation was on a voluntary basis and the respondents did not receive any incentive.

\section{The interview scheme}

An interview scheme with questions about fat intake, psychosocial determinants. interpersonal comparison aspects and demographics was constructed.

Fat intake was measured by means of a validated food frequency questionnaire that assesses frequency, quantity and type (high fat, medium fat, or low fat) of 12 products or product categories that contribute the most to saturated fat intake in the Netherlands (i.e., dairy products, bread spreads, cheese, meat, gravy, savoury snacks and sweet snacks). The answers were used to calculate fat points for each product or product category. These fat points added up to a fat score (ranging from 
12 to 60 ) that reflected total saturated fat intake (Van Assema et al., 1992; Van Assema et al, 2001).

The psychosocial questions assessed 'perceived fat intake' and 'perceived fat intake compared to that of others'. The questions consisted of two items on a fivepoint scale: 'Do you think you eat much or a little fat?' (very much - very little) and 'Do you think you eat more or less fat than others of your age and sex?' (much more - much less). Two new variables, 'awareness of personal fat intake' and 'awareness of personal fat intake compared to that of others'. were constructed by relating the 'perceived fat intake' and the 'perceived fat intake compared to that of others' to the calculated fat intake from the food-frequency questionnaire and the average intake levels of peers (the average intake level of peers was drawn from prior surveys). Respondents who thought to eat a little or very little fat. while the calculated fat points exceeded the cut-off point for the recommended intake level, were classified as underestimators, others as non-underestimators. Respondents who perceived their fat intake as less or much less than that of others of the same age and sex, while the calculated fat points exceeded the cut-off point for the average intake level of peers, were classified as biased comparers, others as non-biased comparers.

Willingness to eat less fat was measured with two items, with yes/no answering options: 'Do you intend to start eating less fat than you do now. within the next 6 months?' and 'Are you currently trying to eat less fat than you did before?' Respondents who answered yes to one or both of these questions were classified as intending to eat less fat, others as not intending to eat less fat.

The questions about interpersonal comparison. which were based on the results of focus group interviews, are listed in Table 7.1. First. four statements about. possible information sources for estimating personal fat intake, including information from comparison with others, were given (Table 7.1. part A). The respondents could indicate whether they did or did not use each type of information for estimating their personal fat intake. The questions regarding comparison targets, i.e., the persons with whom comparisons were made (Table 7.1, part B). were all preceded by briei introductions like: 'Research has shown that people sometimes compare themselves with others to get an idea of how much fat they eat'. The interviewers were instructed to give some explanation to the questions when necessary. The respondents were first asked, in an open-ended question, with whom they compared themselves in terms of fat intake. Subsequently, they were asked to indicate whether they compared themselves with persons specifically mentioned by the in * terviewer: The respondents who had mentioned to compare themselves with one or more of these comparison targets were asked to indicate on five-point scales how much fat (very much - very little) they thought their comparison targets ate. This information was used to assess whether the respondents compared themselves to people whom they perceived to consume small, average or large amounts of fat. To explore what kind of information people tend to use for making interpersonal comparisons, the respondents were asked to indicate on five-point scales to what extent they agreed (completely agree - completely disagree) with statements about (1) obtaining information from particular situations in which interpersonal com- 
A To estimate whether I eat much or a little fat, I compare my food habits with ......

.... My own ideas about how much fat is healthy (completely agree - completely disagree)

.... Nutritional guidelines about healthy amounts of fat (completely agree - completely disagree)

.... How much fat others eat (completely agree - completely disagree)

.... Nutrition information on packages (completely agree - completely disagree)

B Can you mention a person to whom you compare yourself in terms of fat intake sometimes? (Open-ended question)

- In terms of fat intake, do you compare yourself with: partner, relatives/a specific relative, colleagues/fellow students, friends, people in the street, people you see in shops or eating in the street, celebrities (yes, no)

- Do you think your partner, relatives, colleagues/fellow students, friends (depending on those mentioned in the first question) eat much or a little fat? (very much - very little)

C I get an idea of how much fat others eat by:

- Observing how they cook (completely agree - completely disagree)

- Observing what they buy (completely agree - completely disagree)

- Comparing the contents of other people's supermarket trolleys with those of my own (completely agree - completely disagree)

- Observing what they have in stock (completely agree - completely disagree)

- Observing what they eat (completely agree - completely disagree)

- Listening to what they say about their food habits (completely agree - completely disagree)

- Observing what they look like (completely agree - completely disagree)

Note. All scales are five point scales.

parison may occur and (2) obtaining information by observing specific aspects of the comparison target (Table 7.1, part C).

General and demographic information was obtained about household composition (single or two or more persons), regular cooking or shopping for the household, level of education, employment, weight, height and gender.

The whole interview scheme had been pre-tested for comprehensibility and feasibility, and it had been adapted on the basis of the results of the pre-test. Completion of the interview took approximately 15 minutes.

\section{Statistical analyses}

Frequencies were computed to describe the study population in terms of the proportions of respondents who had a diet not in agreement with recommendations. who underestimated their personal fat intake compared to recommended intake levels, who underestimated their personal fat intake compared to that of peers and who intended to change. Univariate binary logistic regression analyses were conducted with intention to change (coded 0 for no intention, 1 for intention) as the dependent and the objectively measured intake level, the subjectively rated intake level and the subjectively rated intake level compared to that of peers as the independent variables. The odds ratios (ORs) and $95 \%$ confidence intervals (Cls) were computed. 
Frequencies were computed to describe (1) the extent to which people reported using any of the possible information sources for estimating personal dietary fat intake, (2) the people who were mentioned as comparison targets and (3) the kind of comparison information that was used and the situations in which comparison information was obtained. Binary logistic regression analyses were used to identify factors that are associated with a tendency to engage in interpersonal comparison with respect to dietary fat intake. Five dependent variables, representing interpersonal comparison in general and comparison with partner, relatives, friends and colleagues specifically (coded 0 for no comparison, 1 for comparison), were separately regressed on a set of independent variables. These variables were gender 10 for male, 1 for female), age, body mass index (BMI), educational level (0 for low, 1 for high), household composition (0 for a single person, 1 for more people), awareness. of personal fat intake (0 for underestimators, 1 for non-underestimators), awareness of personal fat intake compared to that of others $(0$ for biased comparers, 1 for non-biased comparers), and intention to change. All the independent variables were entered in one step. Odds ratios and $95 \%$ confidence intervals were computed. Frequencies were computed to describe whether the specific comparison targets. were perceived to eat small, average or large amounts of fat.

An alpha $<.05$ was determined as the significance level.

\section{Results}

Objectively and subjectively measured intake levels, intention to change and associations - The average intake level of fat was 28 points $(S D=6)$ for the male participants and 24 points $(S D=6)$ for the female participants. Fifty-four percent of the respondents exceeded the fat intake levels of 26 points for men and 23 points for women, which are approximations of recommended intake levels. Forty-two percent of the respondents consumed more fat than the sex-specific mean intake level of 28 points for men and 25 points for women. Forty-two percent underestimated their fat intake and $32 \%$ underestimated their fat intake compared to that of others. Forty-six percent of the respondents intended to reduce their fat intake. There was no significant association between the objectively assessed fat intake and intention to change $(O R=1.02, C I=0.98-1.07)$. However, we found a significant association between self-rated fat intake and intention to change $(O R=1.4, C I=1.03-1.99)$ and a borderline significant association between self-rated fat intake compared to that of others and intention to change $(O R=1.6, C I=0.99-2.43)$.

\section{Do people use interpersonal comparison information to evaluate their fat intake?}

- Only fifteen percent of the respondents indicated that they used information about the fat intake of others as a means of evaluating their own fat intake. The respondents more often indicated using their own ideas about healthy amounts of fat $(76 \%)$, nutrition information on packages $(51 \%)$, and recommendations about fat consumption $(46 \%)$ as information sources in estimating how much fat they 
consumed (Table 7.1. part A).

To whom do people compare their own fat intake? - Seventy-one percent of the respondents, however, reported to compare themselves in terms of fat intake to one or more specific comparison targets. The partner was the most frequently mentioned comparison target (43\%). followed by relatives or a specific relative (36\%). people in the street/in shops (34\%), friends or acquaintances (34\%), colleagues or fellow students (25\%), neighbours (11\%) and celebrities (7\%) (Table 7.1, part B).

What diet-related comparison information do people use? - Eighty-one percent of the respondents indicated that they obtained comparison information by observing what others eat: $71 \%$ by observing what others buy: $67 \%$ by observing what others look like; $64 \%$ by observing how others cook; $54 \%$ by listening to what others say about their food habits: $44 \%$ by observing what products others have in stock: and $37 \%$ by comparing the contents of their own supermarket trolley with those of others (Table 7.1, part C).

Table 7.2. Odds ratios and $95 \%$ confidence intervals from logistic regression analyses identifying factors that are associated with a tendency to engage in different possible interpersonal comparisons

\begin{tabular}{|c|c|c|c|c|c|}
\hline $\begin{array}{l}\text { Dependent } \rightarrow \\
\text { Independent } \\
\downarrow\end{array}$ & $\begin{array}{l}\text { Comparison } \\
\text { in general } \\
(n-183)\end{array}$ & $\begin{array}{c}\text { Comparison } \\
\text { with partner } \\
(n-161)\end{array}$ & $\begin{array}{c}\text { Comparison } \\
\text { with relatives } \\
(n-181)\end{array}$ & $\begin{array}{c}\text { Comparison } \\
\text { with friends } \\
(n-180)\end{array}$ & $\begin{array}{l}\text { Comparison } \\
\text { with colleagues } \\
(n-156)\end{array}$ \\
\hline Gender & $\begin{array}{c}0.98 \\
(0.46-2.10)\end{array}$ & $\begin{array}{c}0.91 \\
(0.44-1.90)\end{array}$ & $\begin{array}{c}1.84 \\
(0.84-4.04)\end{array}$ & $\begin{array}{c}1.09 \\
(0.50-2.37)\end{array}$ & $\begin{array}{c}0.45 \\
(0.19-1.07)\end{array}$ \\
\hline Age & $\begin{array}{c}0.97 \\
(0.94-0.99)\end{array}$ & $\begin{array}{c}0.99 \\
(0.96-1.02)\end{array}$ & $\begin{array}{c}0.97 \\
(0.94-1.00)\end{array}$ & $\begin{array}{c}0.97 \\
(0.94-1.00)\end{array}$ & $\begin{array}{c}0.96 \\
(0.92-0.99)\end{array}$ \\
\hline BM! & $\begin{array}{c}1.00 \\
(0.90-1.10)\end{array}$ & $\begin{array}{c}1.00 \\
(0.92-1.10)\end{array}$ & $\begin{array}{c}0.96 \\
(0.87-1.05)\end{array}$ & $\begin{array}{c}0.99 \\
(0.89-1.09)\end{array}$ & $\begin{array}{c}0.96 \\
(0.84-1.08)\end{array}$ \\
\hline Education & $\begin{array}{c}0.93 \\
(0.45-1.95)\end{array}$ & $\begin{array}{c}1.08 \\
(0.55-2.12)\end{array}$ & $\begin{array}{c}1.03 \\
(0.51-2.09)\end{array}$ & $\begin{array}{c}2.06 \\
(1.01-4.19)\end{array}$ & $\begin{array}{c}1.86 \\
(0.84-4.09)\end{array}$ \\
\hline Family composition & $\begin{array}{c}1.13 \\
(0.45-2.81)\end{array}$ & $\begin{array}{c}1.90 \\
(0.61-5.98)\end{array}$ & $\begin{array}{c}0.84 \\
(0.31-2.27)\end{array}$ & $\begin{array}{c}1.12 \\
(0.40-3.13)\end{array}$ & $\begin{array}{c}0.97 \\
(0.32-2.95)\end{array}$ \\
\hline $\begin{array}{l}\text { Awareness of per- } \\
\text { sonal fat intake }\end{array}$ & $\begin{array}{c}0.74 \\
(0.34-1.65)\end{array}$ & $\begin{array}{c}0.98 \\
(0.48-1.98)\end{array}$ & $\begin{array}{c}1.29 \\
(0.61-2.74)\end{array}$ & $\begin{array}{c}1.20 \\
(0.56-2.59)\end{array}$ & $\begin{array}{c}0.85 \\
(0.36-2.02)\end{array}$ \\
\hline $\begin{array}{l}\text { Awareness of } \\
\text { personal fat intake } \\
\text { compared to that of } \\
\text { others. }\end{array}$ & $\begin{array}{c}0.70 \\
(0.28-1.78)\end{array}$ & $\begin{array}{c}1.73 \\
(0.78-3.82)\end{array}$ & $\begin{array}{c}0.82 \\
(0.36-1.85)\end{array}$ & $\begin{array}{c}0.63 \\
(0.28-1.43)\end{array}$ & $\begin{array}{c}1.23 \\
(0.48-3.13)\end{array}$ \\
\hline $\begin{array}{l}\text { Intention to eat } \\
\text { less fat }\end{array}$ & $\begin{array}{c}2.27 \\
(1.06-4.82)\end{array}$ & $\begin{array}{c}1.20 \\
(0.61-2.37)\end{array}$ & $\begin{array}{c}4.38 \\
(2.13-8.99)\end{array}$ & $\begin{array}{c}3.22 \\
(1.57-6.61)\end{array}$ & $\begin{array}{c}2.73 \\
(1.19-6.28)\end{array}$ \\
\hline
\end{tabular}

Note. The table should be read in columns. Each column includes the dependent variable with the set of independent variables in the equation, For dummy coding, see 'Statistical analyses' in the 'Methods' section. Bold values indicate statistical significance of the value. 
Table 7.3. Participants perceptions of the fat intakes of four specific comparison targets, in terms of small, large or average amounts of fat

\begin{tabular}{lcccc}
\hline & \multicolumn{4}{c}{ Comparison with } \\
\cline { 2 - 5 } Intake perceived as & $\begin{array}{c}\text { Partner } \\
(n-70)\end{array}$ & $\begin{array}{c}\text { Relatives } \\
(n-67)\end{array}$ & $\begin{array}{c}\text { Friends } \\
(n-56)\end{array}$ & $\begin{array}{c}\text { Colleagues } \\
(n-37)\end{array}$ \\
\hline Small & $43 \%$ & $30 \%$ & $13 \%$ & $24 \%$ \\
Largeb & $30 \%$ & $33 \%$ & $41 \%$ & $43 \%$ \\
Averagé & $27 \%$ & $37 \%$ & $46 \%$ & $32 \%$ \\
\hline
\end{tabular}

Note. "The respondent indicated that the comparison target consumed a little, or very little, fat; "The respondent indicated that the comparison target consumed much, or very much, fat; 'The respondent indicated that the comparison target consumed an average amount of fat.

Factors associated with a tendency to engage in interpersonal comparison - Respondents who intended to eat less fat and younger respondents were significantly more likely to compare themselves with others in general and with colleagues or fellow students (Table 7.2). Respondents with a higher educational level and those who intended to eat less fat were more likely to compare themselves with friends. Respondents who intended to eat less fat were also more likely to compare themselves with relatives.

Do people compare themselves with others whom they perceive to eat small, average or large amounts of fat? - Partners were dominantly perceived as consuming small amounts of fat, whereas the perceived amount for relatives was equally distributed among small, large or average amounts (Table 7.3). Friends' consumption levels were predominantly perceived as average or high, and those of colleagues as high.

\section{Discussion}

The present study was a preliminary exploration of the occurrence and nature of interpersonal comparison regarding dietary fat intake. The results indicate that comparison with others is indeed used to evaluate personal dietary fat intake, although this is only revealed by focussing on specific comparison targets or comparison situations and not from general self-reports about the use of interpersonal comparison information. It seems possible to identify people who are more likely than others to be chosen as comparison targets and to recognize sources for obtaining comparison information that are more frequently used than others. It also seems possible to distinguish people who are more likely than others to make interpersonal comparisons of dietary fat intake.

The Social Comparison Theory (SCT) (e.g., Buunk \& Gibbons, 1997; Festinger, 1954: Suls \& Wheeler, 2000; Wood. 1989) posits that people have a natural urge 
to evaluate their opinions, abilities and performances, preferably in relation to an objective standard. If no objective standard is available, people will use less objective information such as social comparison information to evaluate their opinions. abilities and performances. Social comparisons will most likely be made with similar others, i.e., people who are similar in terms of age, gender, lifestyle, and so on. Social comparison can serve several goals, such as improving a particular ability or performance (self-improvement), feeling good about oneself (self-enhancement) and getting accurate information about a particular personal ability or performance (self-evaluation). The finding that the occurrence of interpersonal comparison became more obvious from the answers to specific questions than from general self-reports may reflect that respondents were hesitant to admit that they compare themselves to others, or that they were not readily aware of the interpersonal comparisons they make. The hesitation to admit comparing oneself to others has been found in earlier studies as well, and it appears to be a common feature in studies of social comparison based on self-reports (cf. Wood, 1996). Wood and colleagues (Wood, Taylor, \& Lichtmann, 1985), for example, found that most people's first reaction when they were interviewed was to say that they do not compare themselves to others. Later in the interview, however, respondents spontaneously described situations that clearly indicated social comparisons.

In line with predictions of the SCT (e.g., Wood, 1989), the respondents in our study compared themselves mostly to their partners, their relatives and their friends, i.e.. people who are quite close to them and who may be quite similar to them. Celebrities, although often used in advertising, were not very likely to be mentioned as comparison targets by our respondents. Most celebrities may be perceived as dissimilar to the self, and according to the SCT, comparison with such dissimilar others is unlikely to occur.

The results of the present study indicate that interpersonal comparison occurs with targets who are perceived to eat small amounts of fat, which may make them positive role models, as well as with targets who are perceived to eat large amounts of fat, i.e., negative role models. The closest people (partners) seem to be more often perceived as consuming small amounts of fat, whereas more distant persons (colleagues) are more likely to be perceived as consuming large amounts of fat. On the basis of the present study, however, we cannot determine what the effects of comparison with positive or negative models on fat intake or determinants of fat intake will be. Future studies should examine this.

The present study was initiated based on the assumption that comparison to others would lead to biased estimates of personal intake, causing a low motivation to change. However, respondents who expressed an intention to eat less fat were found to be more likely to report that they compared themselves to others than respondents with no intention to change. Earlier studies have found that people are more likely to engage in social comparison activities in periods of change or uncertainty (cf. Gibbons \& Buunk, 1999). Furthermore, for people who intend to change, eating less, fat is more likely to be a topic of personal interest, which may lead them to more deliberately seek and process information regarding dietary fat 
intake. This may include social comparison information (Petty \& Cacioppo, 1986).

The SCT distinguishes three motives for social comparison: self-improvement, self-evaluation and self-enhancement (Wood, 1989). Our data suggest that it is especially people who intend to change who compare themselves with others. Self-improvement may therefore be the primary motive for comparison in the population we studied. This finding may be interesting for nutrition education interventions. People who intend to change may be the ones most receptive to interventions that use social comparison information. The best-known intervention strategy based on comparisons with others is modelling or observational learning (Bandura, 1986). Role models are traditionally presented as targets who perform better than the intended observers, in that they are well-skilled. possess the desired attitudes and engage in the desired behaviour. These better-performing models may be appropriate for people who intend to change. In other phases of the behavioural change process, however, people may be more interested in comparison with others who perform equally well or slightly worse (e.g.. Tigges, 2001). Thus, insight into interpersonal comparison regarding dietary fat intake may make it possible to find appropriate comparison targets for people in different phases of the behavioural change process.

There are some limitations to the present study. The study was cross-sectional, so no causal inferences can be made. Data were collected retrospectively, which means that recalling interpersonal comparison situations may not have been accurate and that respondents who were not aware of having made interpersonal comparisons in the past may not have been able to recall them at all. The present study was based on a random sample of the Dutch population, but non-response may have led to a selection bias. Nevertheless, the proportion of respondents who exceeded the recommended intake levels were similar to the Dutch adult population at large (Voedingscentrum, 1998). Despite its limitations, the present study contributes to a basic understanding of the occurrence and nature of interpersonal comparisons that are made regarding dietary fat intake. 


\section{An exploration of the effects of positive and negative role model stories on awareness, intention and fruit intake}

\section{Abstract}

There is a need for health education interventions to motivate people to adopt more healthful diets. Lack of awareness of personal dietary intake is a barrier for the intention to change, and interventions should be developed aimed at increasing awareness. Social comparison information was explored for its usefulness and efficacy in increasing awareness, motivation and behaviour change related to fruit intake. Social comparison information was provided through positive and negative role model stories. The study was conducted as a randomised controlled trial, with a three-conditions-between-subjects design. Post-test measurements were immediately and two weeks after the intervention. Respondents were undergraduate university students $(N=132)$, who had lower than recommended fruit intake levels. No significant group effects were found on any of the outcome measures. at both post-tests. There was more identification with the model and greater perceived credibility of the information in the negative role model group. Despite the lack of effects on the outcome measures, there are some implications that may be of relevance for health education practice. More research in use and application of the principle of social comparison information in health education interventions is needed. 


\section{Introduction}

There is compiling evidence that diets low in saturated fat and high in fruit and vegetables may contribute to the prevention of cardio-vascular diseases, obesity. non-insulin dependent diabetes and certain types of cancer (Joshipura et al., 2001: Key et al., 2002: Rolls \& Shide, 1992; Willet, 1994). Nevertheless, in most Western countries many people eat more saturated fat and less fruit and vegetables than recommendations and many of them do not intend to change (Beer-Borst et al. 2000; Ganji \& Betts, 1995: Kearney, Gibney, Martinez, De Almeida, Friebe, Zunft. Widhalm. \& Kearney, 1997: Van Wechem et al., 1998; Voedingscentrum, 1998). Therefore, there is a need for health education interventions that are able to motivate people to change to more healthful diets and to achieve actual changes in dietary practices. The present study aims to explore the usefulness and efficacy of social comparison information as a strategy in promoting healthy dietary behavfour. Based on insights from modelling (Bandura, 1986) and Social Comparison Theory (Festinger, 1954; Wood, 1989), social comparison information was provided in positive and negative role model stories. This study explored the effects of both types of role model stories on fruit intake. Fruit intake was selected as a target behaviour, since fruit is generally considered to be an important ingredient for a healthy diet and because the recommended intake of fruit is clearly defined as two pieces per day. This facilitated the development of role model stories from which the target behaviour could be interpreted clearly and unambiguously.

\section{Role model stories as a possible strategy to improve fruit intake}

Lack of awareness of personal dietary intake has been associated with a low motivation to change to a more healthful diet (Brug et al., 1994; Glanz et al., 1997). People who are not aware of their own dietary intake will not perceive a possible need to change, will be less likely to intend to change and may not be likely to be attentive to healthy diet promotion information. In terms of the Precaution Adoption Process Model (Weinstein \& Sandman, 1992; Weinstein \& Sandman, 2002). people who are not aware of their personal dietary intake are in the so-called unengaged stage. People in that stage know that unhealthful diets may pose a risk to health. They may also know that many other people have unhealthful diets. However, they do not acknowledge to consume unhealthful diets themselves (Weinstein, 1988). In order to become motivated to change and proceed to action, these people first need to become aware of their own unhealthful diet. Thus, it is important to develop interventions aimed at increasing awareness. An optimistic bias in the perception of one's own risk behaviour compared to that of others, has found to be associated with lack of awareness of dietary intake (Brug et al., 1998b). That is, the more fruit people think to eat, compared to others, the more they overestimate their fruit intake. Providing social comparison information, i.e., information about the behaviour of a particular peer, could be a way to modify perceptions of one's own fruit intake and induce an intention to change. Role model stories are a well- 
accepted way to provide this kind of social comparison information.

Role model stories most often present positive role models (models who perform better than the intended observers) to induce change. According to modelling theory a role model should be attractive for the intended observers and the modelled behaviour should be perceived as relevant and as rewarding (Bandura, 1986). However, social comparison theory posits that negative role models (models who perform worse than the intended observer) may also induce motivation and behaviour change. Providing social comparison information may be regarded as a form of performance feedback (Klein, 2003). A person who is exposed to role model information may infer that he or she is doing better, equally well, or worse than the role model. Providing a positive role model may be perceived as negative feedback by the observer, whereas providing a negative role model may be perceived as positive feedback. This positive feedback (induced by a negative model) may lead to motivation and action, whereas negative feedback (induced by a positive model) could also lead to motivation and behaviour change. Negative feedback may, however. also put self-evaluation maintenance strategies in effect, such as downgrading the accuracy of the information or downgrading the personal importance of performing a particular behaviour (Klein, \& Weinstein, 1997: Klein, 2003). In conclusion, positive role models may not necessarily or not always induce motivation and behaviour change, and negative models may well be able to induce motivation and behaviour change. Therefore, it is worth to explore both positive and negative role model stories for their potential to be included in interventions aimed at inducing healthy diets.

The aim of the present study is to explore the effects of positive and negative role model stories on awareness, intentions to change and intakes of fruit, among students with a fruit intake lower than the recommended minimum of two pieces of fruit a day. The effects of the role model stories were compared to a control group that received a role model story irrelevant to fruit consumption.

\section{Methods}

\section{Design and participants}

The study was conducted as a randomised controlled trial, with a three-conditionsbetween-subjects design (positive role model story vs. negative role model story vs. control). Measurements were at baseline and immediately and two weeks after exposure to the role model information. Participants were first-year undergraduate students in health sciences at Maastricht University, in their first semester. Of the 350 students who were invited for the study, 313 participated. Of these students 132 (34\%) were identified as having fruit intakes below the recommended intake level and those students were included in the study. Nine respondents were excluded from the analyses because of incomplete data sets. Forty respondents remained in the positive model group, 39 in the negative model group and 44 in the 
control group. Response-rate at post-test 2 was $55 \%$ in the positive model group. $69 \%$ in the negative model group and $48 \%$ in the control group.

\section{Procedure}

Students were informed about the study and invited to participate during a lecture, which was attended by approximately 350 students. The study was conducted during regular study group meetings, consisting of 10 to 12 students and a tutor. The study materials were distributed through the tutors, who were instructed on when and how to distribute the materials. Participants randomly received either positive or negative gender specific role model stories (see materials). Each student received a package containing the baseline questionnaire, an envelope with the comparison information (marked as 1), and an envelope with the first post-test questionnaire (marked as 2). Students were instructed to start with completing the baseline questionnaire that ended with the instruction to open envelope 1 and read the information thoroughly. Subsequently, respondents were instructed to open envelope 2 and complete the post-test questionnaire. The tutors took care for an orderly and quiet atmosphere and for the completion of the study elements in. the right order. All materials were handed in to the tutor and were subsequently collected by the researchers. Completing the questionnaires and reading the information took about 20 minutes. Two weeks later, the students were sent an e-mail with a link to the second post-test questionnaire that could be completed online. Respondents who did not return this questionnaire within the requested time were sent a reminder e-mail. After the study was completed, respondents were debriefed through a letter sent by e-mail.

\section{Materials}

The interventions consisted of role model stories in printed form. The stories were compiled from real ilfe interviews with first-year students conducted by the researcher. There were female and male versions of each model story, which were provided to female and male students correspondingly. Taken the female version as an example, the role model siories were as follows. The student first introduced herself and told about how it was to start studying in Maastricht. Then she told about how much fruit she consumed and the reasons to do that. The positive role model told that she consumed at least two or three pieces of fruit each day, because it was tasty, healthy, provided energy, prevented weight gain, that it was easy to eat fruit and how she managed to eat sufficient amounts of fruit every day. She met the Dutch guideline of eating iwo pieces of fruit each day. The negative role model told that she did not eat fruit very often. consuming a maximum of two pieces of fruit a week, and sometimes less. She rather ate something else than fruit, easily forgot to: eat fruit and thought it was inconvenient to eat. She thought that it was healthy to eat fruit, however acknowledged that she did not meet the guideline of two pieces of fruit a day. The model in the control condition told about what social and sports. 
activities she planned to do in Maastricht. All stories were similar in length.

\section{Measurement}

Fruit consumption was measured using a validated short fruit consumption questionnaire that assessed the frequency and amount of intake of fruit juice and six main categories of fruit (Bogers et al., accepted; Van Assema et al. 2002). The average intake of pieces of fruit per day was computed from this questionnaire. Selfrated intake and self-rated intake compared to others was measured using single items ['how much fruit do you think you eat' and 'how much fruit do you think you eat compared to others of your age and sex'] on five point scales ['very little - very much" and 'much less - much more']. These ratings, which were statistically adjusted for objectively assessed intake, were used as an indicator for awareness (Brug et al. 1998b). Intention to eat more fruit and intention to eat at least two pieces of fruit per day were measured with single items on five point scales ['do you intend to eat more fruit than you do now' and 'do you intend to eat at least two pieces of fruit per day', with answering options 'surely do not - surely do']. Fruit intake was measured at baseline and at the second post-test, while self-rated intake and intentions were measured in all three measurements. Gender, age, height and weight were assessed at baseline.

The first post-test included additional questions to check the successfulness of the manipulation. comparison and identification with the role model and evaluation of the intervention. The manipulation was checked in the experimental groups by means of the question. 'Do you think the model performed better or worse than you do with respect to fruit intake?'. Two questions assessed the extent to which the respondent compared him/her self to the model. I'To what extent did you compare yourself with the model' and, 'To what extent did you compare yourself with the model concerning fruit intake' (the latter was only asked in the experimental groups)]. Three questions assessed identification with the model, ['Did you recognise something of yourself in the model'. 'Do you think you are comparable with the model' and 'Did you see similarities between yourself and the model'l. Finally, use and appreciation of the information was assessed with several items, I'How thoroughly did you read the information' and 'How enjoyable/interesting/credible did you think the information was']. All items had five point bipolar answering scales with end labels that corresponded with the specific question (e.g., very uninteresting - very interesting).

\section{Statistical analyses}

Binary logistic regression analyses were conducted to identify possible predictors for drop out at post-test 2. Drop out was regressed on group. gender, body-mass index and baseline measures of fruit intake, self-rated fruit intake, self-rated fruit intake compared to others, intention to eat more fruit and intention to eat two pieces of fruit per day. Logistic regression analyses with group as the dependent 
variable and the before-mentioned independent variables minus group, were conducted to examine the successfulness of the randomisation.

Linear regression analyses were conducted to examine associations between intentions, fruit intake and self-rated fruit intake. Intentions were regressed on fruit intake and self-rated fruit intake in univariate regression analyses, and on fruit intake and self-rated fruit intake combined in multiple regression analyses. A binary logistic regression analysis was conducted to examine the association between over-estimation of fruit intake and comparison with others. A measure for over-estimation of fruit intake was computed, by classifying respondents who rated their fruit intake as neither a lot or a little, or a lot as optimists (coded 1). others as non-optimists (coded 0 ). This variable for over-estimation was regressed on self-rated fruit intake compared to others.

Analyses of variance were conducted to check the successfulness of the manipulation and to detect group differences in comparison and identification with the role model, the extent to which the information was read and evaluative ratings. Multiple linear regression analyses were conducted to test group differences in outcome measures at post-test 1 and at post-test 2 separately. Two dummy variables for group were computed, with the control group as the reference. The analyses included the post-test score of the outcome measures (self-rated fruit intake, selfrated fruit intake compared to others, intention to eat more fruit. intention to eat two pieces of fruit per day and fruit intake) as the dependent variable and the dummies for group and the baseline measure of the outcome indicator as the independent variables. In the analyses on self-rated intake and self-rated intake compared to others at post-test 1, baseline fruit intake was added as an independent variable to create the indicator for awareness (i.e., self-rated intake adjusted for 'real' intake (Brug et al. 1998b)). To create an indicator for awareness at post-test 2, fruit. intake at post-test 2 was included as an independent variable in the analyses on self-rated intake and self-rated intake compared to others. An intention-to-treat procedure was employed, to account for drop out at post-test 2.

\section{Results}

\section{Participants}

Mean age of the respondents was 19 years $(S D=2)$ and $72 \%$ were female. The average intake of fruit was 1.2 pieces per day $(S D=0.45)$. Forty-six percent of the respondents over-estimated their fruit intake. Sixty-four percent had a positive intention toward increasing fruit intake and $39 \%$ had a positive intention toward eating two pieces of fruit per day. Respondents who dropped out were significantly less likely to be in the downward comparison group than in the upward comparison group (Odds Ratio $[O R]=0.27,95 \%$ confidence interval $[C]=0.10-0.77$ ) or the control group $(O R=0.20, C I=0.07-0.56)$. Furthermore, drop out was associated with fruit intake. The group that dropped out had a higher average fruit intake 
Table 8.1. Standardised regression coefficients ( $B$ values) for assessed fruit intake and self-rated fruit intake in univariate and multiple linear regression analysis

\begin{tabular}{llcc}
\hline & & Univariate & Multiple \\
\hline Intention to eat more fruit & Fruit intake & -11 & .01 \\
& Self-rated intake & $-.24 * *$ & $.24 *$ \\
Intention to eat two pieces of fruit per day & Fruit intake & $.24 * *$ & .00 \\
& Self-rated intake & $.44 * \cdots$ & $.44 * *$ \\
\hline
\end{tabular}

${ }^{*} p<0.5, \cdots p<.01, \cdots p<.001$

than the group that did not drop out $(O R=4.14, C I=1.42-12.10)$. The test for successfulness of the randomisation revealed that baseline fruit intake was higher in the negative model group $(O R=4.49, \mathrm{CI}=1.25-16.18)$ than in the positive model group.

\section{Associations between intentions, fruit intake and self-rated fruit intake}

Fruit intake was not significantly associated with the intention to eat more fruit (Table 8.1), however self-rated fruit intake was, both in the univariate and the multiple regression analyses. The negative association indicates that a higher selfrated fruit intake was associated with a lower intention to change.

Fruit intake was significantly associated with the intention to eat at least two pieces of fruit each day, but only in the univariate analysis. Self-rated fruit intake was significantly positively associated with the intention to eat at least two pieces of fruit per day in the univariate and multiple regression analyses, indicating that a higher self-rated intake was associated with a greater intention to eat two pieces of fruit each day. There was a significant association between optimism and selfrated intake compared to others $(O R=6.0, C I=2.9-12.1)$. The greater the amount of fruit respondents thought to eat compared to others, the more likely they were to over-estimate their intake.

\section{Manipulation check, comparison with the model and identification with the model}

The positive role model was perceived as performing better than the receiver with respect to fruit intake, whereas the negative role model was perceived as performing worse (Table 8.2). The performance ratings differed significantly between the groups. Although there was a certain level of comparison with the role model, especially with respect to fruit intake, there was no difference between the groups in the extent to which the respondents compared themselves with the role model. The negative model was perceived as significantly more comparable, more similar and providing more recognition of the self than the positive model. 
Table 8.2. Means (SD) for manipulation check and extent of comparison and identification with the role model, $F$-value and Tukey post hoc comparison test

\begin{tabular}{|c|c|c|c|c|c|}
\hline & $\begin{array}{c}\text { PM } \\
(n-40)\end{array}$ & $\begin{array}{c}\text { NM } \\
(n=39)\end{array}$ & $\begin{array}{c}C \\
(n-44)\end{array}$ & $F(d f)$ & Post Hoc \\
\hline $\begin{array}{l}\text { Perceived performance of } \\
\text { the role model concerning } \\
\text { fruit intake }\end{array}$ & $1.40(0.71)$ & $-0.69(0.89)$ & & $\begin{array}{l}65.82^{\cdots} \\
(2,76)\end{array}$ & $\mathrm{PM}>\mathrm{NM}$ \\
\hline $\begin{array}{l}\text { Extent of comparison with } \\
\text { model concerning fruit } \\
\text { intake }\end{array}$ & $0.48(1.26)$ & $0.62(1,16)$ & & $\begin{array}{r}0.13 \\
(2,76)\end{array}$ & ns \\
\hline $\begin{array}{l}\text { Extent of comparison with } \\
\text { model in general }\end{array}$ & $0.05(1.13)$ & $0.28(1.23)$ & $-0.09(1.07)$ & $\begin{array}{c}1.11 \\
(2,120)\end{array}$ & ns \\
\hline $\begin{array}{l}\text { Perceived comparablity to } \\
\text { role model }\end{array}$ & $-0.53(1.06)$ & $0.10(1.19)$ & $0.05(0.99)$ & $\begin{array}{r}4.17^{*} \\
(2,120)\end{array}$ & $\mathrm{PM}<\mathrm{NM}, \mathrm{C}$ \\
\hline $\begin{array}{l}\text { Perceived similarity with } \\
\text { role model }\end{array}$ & $-0.40(1.23)$ & $0.26(1.23)$ & $0.00(0.94)$ & $\begin{array}{r}3.85^{\circ} \\
(2,120)\end{array}$ & $\mathrm{PM}<\mathrm{NM}$ \\
\hline $\begin{array}{l}\text { Recognition of self in role } \\
\text { model }\end{array}$ & $-0.20(1.07)$ & $0.59(1.27)$ & $0.30(1.05)$ & $\begin{array}{r}4.95 \cdots \\
(2,120)\end{array}$ & $\mathrm{PM}<\mathrm{NM}$ \\
\hline
\end{tabular}

Note. PM - positive model group, NM - negative model group, C - control group. All scales ranged from -2 to $+2{ }^{*} p<.05, \cdots p<.01, \cdots p<.001$.

\section{Evaluation}

In all three study groups the information provided in the model stories. was rated as equally neutral for importance and enjoyability and equally negative for interestingness (Table 8.3 ). The credibility of the role model story presented in the positive model group was rated significantly lower than in the other groups. There were no significant differences in thoroughness of reading the information.

\section{Awareness, intention to change and intake}

There were no significant group effects on any of the outcome measures at both post-tests, except for a borderline significant group effect for the positive model group on self-rated fruit intake at post-test 2 (Table 8.4). Respondents in the positive model group had a higher level of awareness than those in the control group.

\section{Discussion}

The present study did not illustrate significant effects of positive or negative role model stories on awareness of personal fruit intake. intention to change and fruit consumption. Stronger identification with the model and greater perceived credibility of the information was found for the negative role model story. 
Table 8.3. Mean (SD) for evaluation of the intervention materials, F-value and Tukey post hoc comparison test

\begin{tabular}{|c|c|c|c|c|c|}
\hline$\sqrt{2}+x^{2}$ & $\begin{array}{c}\text { PM } \\
(n-40)\end{array}$ & $\begin{array}{c}\text { NM } \\
(n-39)\end{array}$ & $\begin{array}{c}C \\
(n-44)\end{array}$ & $F(d t)$ & Post Hoc \\
\hline Perceived credibility of story & $0.33(1.27)$ & $1.37(0.67)$ & $1.02(0.82)$ & $\begin{array}{c}12.18 \cdots \cdots \\
(2,119)\end{array}$ & $\mathrm{PM}<\mathrm{NM}, \mathrm{C}$ \\
\hline $\begin{array}{l}\text { Perceived importance of } \\
\text { subject }\end{array}$ & $0.08(0.90)$ & $0.03(0.87)$ & $-0.02(0.85)$ & $\begin{array}{c}0.14 \\
(2,119)\end{array}$ & ns \\
\hline $\begin{array}{l}\text { Perceived attractiveness of } \\
\text { information }\end{array}$ & $-0.20(0.85)$ & $-0.37(0.91)$ & $-0.02(0.66)$ & $\begin{array}{c}1.86 \\
(2,119)\end{array}$ & ns \\
\hline Enjoyability & $0.00(0.91)$ & $0.05(0.86)$ & $0.18(0.69)$ & $\begin{array}{c}0.56 \\
(2,120)\end{array}$ & ns \\
\hline $\begin{array}{l}\text { Thoroughness of reading the } \\
\text { information }\end{array}$ & $0.64(0.84)$ & $0.74(0.72)$ & $0.50(0.76)$ & $\begin{array}{c}1.04 \\
(2,119)\end{array}$ & ns \\
\hline
\end{tabular}

Note. PM - positive model group, NM - negative model group, C - control group. All scales ranged from -2 to $+2 \cdots p<.001$.

Table 8.4. Means (SD) of the outcome measures for each group at baseline, post-test one and posttest two. Standardised regression coefficient (B) for group from the regression analyses, with the control group as the reference

\begin{tabular}{|c|c|c|c|c|c|}
\hline & \multirow{2}{*}{$\begin{array}{c}\text { Positive } \\
\text { model group } \\
(n-40)\end{array}$} & \multirow{2}{*}{$\begin{array}{c}\text { Negative } \\
\text { model group } \\
(n-39)\end{array}$} & \multirow{2}{*}{$\begin{array}{l}\text { Control } \\
\text { group } \\
(n-44)\end{array}$} & \multicolumn{2}{|c|}{ B } \\
\hline & & & & PM & NM \\
\hline \multicolumn{6}{|c|}{ Self-rated intake compared to others ${ }^{a}$} \\
\hline Baseline & $-0.23(0.73)$ & $-0.08(0.94)$ & $-0.32(0.98)$ & & \\
\hline Post-test 1 & $-0.30(0.76)$ & $-0.15(0.84)$ & $-0.48(0.95)$ & 0.06 & 0.07 \\
\hline Post-test 2 & $-0.33(0.84)$ & $-0.11(0.86)$ & $-0.34(0.96)$ & -0.03 & 0.02 \\
\hline \multicolumn{6}{|c|}{ Self-rated intake } \\
\hline Baseline & $-0.58(0.98)$ & $-0.55(1.08)$ & $-0.77(0.99)$ & & \\
\hline Post-test 1 & $-0.48(0.85)$ & $-0.49(1.02)$ & $-0.68(0.98)$ & 0.05 & -0.01 \\
\hline Post-test 2 & $-0.67(1.00)$ & $-0.45(0.98)$ & $-0.64(0.97)$ & $-0.10^{\circ}$ & 0.00 \\
\hline \multicolumn{6}{|c|}{ Intention to eat more fruit" } \\
\hline Baseline & $0.73(0.91)$ & $0.64(1.11)$ & $0.70(1.00)$ & & \\
\hline Post-test 1 & $0.77(0.92)$ & $0.62(1.21)$ & $0.61(0.89)$ & 0.07 & 0.02 \\
\hline Post-test 2 & $0.47(1.01)$ & $0.49(1.17)$ & $0.48(1.02)$ & -0.01 & 0.03 \\
\hline \multicolumn{6}{|c|}{ Intention to eat two pieces of fruit" } \\
\hline Baseline & $0.13(1.14)$ & $0.15(1.23)$ & $-0.05(1.22)$ & & \\
\hline Post-test 1 & $0.47(0.99)$ & $0.26(1,12)$ & $0.34(0.91)$ & 0.01 & -0.10 \\
\hline Post-test 2 & $-0.05(0.18)$ & $0.10(1.23)$ & $-0.05(1.10)$ & -0.06 & -0.01 \\
\hline \multicolumn{6}{|l|}{ Fruit intake } \\
\hline Baseline & $1.09(0.45)$ & $1.33(0.43)$ & $1.18(0.51)$ & & \\
\hline Post-test 1 & - & - & - & & \\
\hline Post-test 2 & $1.28(0.64)$ & $1.37(0.57)$ & $1.28(0.52)$ & 0.07 & -0.04 \\
\hline
\end{tabular}

Note. PM - standardised regression coefficient for the positive model group NM - standardised regression coefficient for the negative model group. "Scales ranging from -2 to +2 , "pieces of fruit. " Borderline significant effect $p=.075$. 
Providing social comparison information through positive role models i.e., modelling) is quite common in health education, including nutrition education. The popularity of modelling is understandable, since in theory, the method of modelling seems to be able to influence many determinants, such as risk perception, skills, knowledge and social influence (Bartholomew et al., 2001). Furthermore, there are many and appealing ways to provide role models and role model information, such as printed role model stories or real-life, video or cyber role models and role model information. Despite the extensive use of modelling in nutrition education interventions, there is hardly any literature on the effects of providing role model stories as such. This may be due to the fact that the effects of role model stories on determinants and behaviour have seldom been studied in isolation, but have mainly been used as a component in multi-method interventions. The present study aimed to explore the mere effect of modelling. However, modelling did not have an effect among this study population on changing fruit intake and its determinants. Due to the lack of published studies, it is difficult to relate the present results to other studies. Published studies using role model stories as an intervention strategy are mainly in the area of HIV/AIDS-prevention and used combinations with other. strategies, which limits the comparability to our study. A study that tested the effects of a role model story in the domain of genetic counselling for cystic fibrosis found modest effects. This study consisted of a quasi-experiment comparing a brochure with traditional information and a role model story to a brochure with traditional information only. Effects were found for the brochure with the role model story on perceived risk and severity of the disease and on perceived barriers, but not in uptake of carrier testing for cystic fibrosis (Leonard, Bartholomew, Swank, \& Parcel. 1995).

Providing social comparison information through a negative role model is new in the field of nutrition education, but some evidence suggests that negative role models may also motivate to take action (Klein. 2003; Lockwood, Jordan, \& Kunda, 2002). The present study did not find such an effect.

The absence of an effect may be a preliminary indication that providing social comparison information in the form of positive or negative role model stories is not an effective strategy to induce changes in fruit intake and its determinants, among students with lower than recommended fruit intake levels. However, there are also some methodological issues in this study that may have contributed to the lack of effects.

The final study sample was quite small. A large effect size would have been needed to detect any effects. with this small sample size. Such a large effect may not to be expected from role model stories alone. Self-rated intake in the positive model group shows a trend toward significance and in a larger sample this may have reached significance. The less than optimal success of the randomisation may have been of influence to the effects found for fruit intake. even though we adjusted the analysis on fruit intake for the baseline fruit intake level.

An intention-to-treat procedure was employed to compensate the bias that may have been introduced by the high and selective drop out at the second post-test. 
However, by doing so, possible effects may have been diluted. Reasons for drop out were not systematically studied. A possible reason may be lack of interest in the topic of fruit intake, as indicated by the neutral perceived importance of the topic. However, perceived importance could not be identified as a predictor for drop out.

A low rating of importance of the topic as well as the neutral to negative ratings for attractiveness and enjoyability of the role model stories may, however, pose a problem in another respect. First of all, a low level of importance, attractiveness and enjoyablity of the information may have falled to attract sufficient attention to the message. Secondly, positive and negative role model stories may be most likely to produce an effect when the topic is of interest and of relevance for a person (Bandura, 1986; Lockwood et al., 2002). Important prerequisites for the role model stories to be effective may not have been met sufficiently. Making the topic more personally relevant and important and making the role model information more attractive may improve the quality of the role model stories, which may make them more successful in producing an effect.

An interesting finding is that there was more identification with the negative role model than with the positive role model. This is, however, commonly found in research on upward and downward comparison information for persons who do not perform well on a certain aspect (e.g., Buunk, Ybema, Gibbons, \& Ipenburg, 2001). This may teach us that people who engage in health risk behaviour (the people who should profit most of it) are not necessarily attracted to positive role model stories.

The aim of the present study was to examine the effects of positive and negative role model stories on awareness of personal fruit intake, intention to change and fruit intake. Despite the lack of an effect on the outcome measures and the methodological limitations, we think that the study incorporates some interesting aspects. When a topic is not of personal interest or relevance for an individual, effects of a modelling intervention may be limited. Persons who engage in health-risk behaviour are not necessarily motivated or attracted to a model that is performing the desired behaviour. More research is needed into how to make derivations and useful applications of insights from social comparison/social cognition research for nutrition education interventions. This may increase the opportunities for developing more powerful interventions based on the principle of social comparison. 


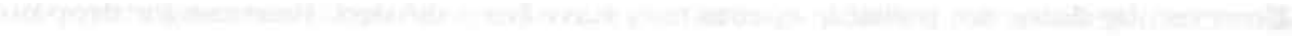

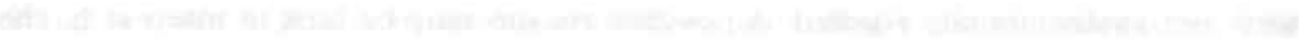

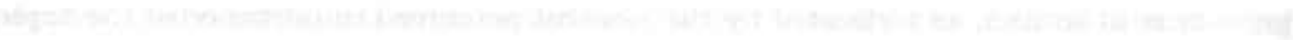




\section{General discussion}

In this thesis intervention strategies to decrease saturated fat intake and increase fruit and vegetable intake were evaluated. The interventions focussed on increasing awareness of personal intake of fat, fruit and vegetables since this was found to be an important barrier for the intention to change (Chapter 1). Increasing awareness would, therefore, be an important step toward behaviour change. Feedback was identified as a method for increasing awareness and was provided through printed self-test forms and through web-based computer-tailored nutrition information. The role of social comparison in dietary intake was explored. and social comparison information in the form of role model stories was investigated for its potential to induce changes in awareness, intentions and behaviour. The interventions were first evaluated in a small-scale controlled setting. The intervention that produced effects in this preliminary evaluation was subjected to a next phase of evaluation in which short and longer-term effects were examined. In this chapter, the results of the studies presented in this thesis will be summarised and integrated, methodological and intervention related issues will be discussed as well as implications for practice and future research. 


\section{Summary and integration of the results of the studies}

\section{Efficacy of self-test forms}

Self-test forms are a brief and easy to administer self-diagnostic tool, through which feedback can be provided. Self-test forms were chosen as a strategy to provide feedback, since, because of its briefness, they may appeal to population groups that are not interested in extensive reading. Furthermore, self-test forms are relatively easy to distribute among large groups of people. A possible disadvantage of self-test forms is that only brief feedback can be provided, based on a rough assessment.

The test of the immediate effects of existing and already widely distributed selftest forms revealed no effect on awareness or intention to change, compared to generic nutrition information (Chapter 4). Respondents' evaluations of the self-test forms were generally similar to that of the generic nutrition information. Since the self-test forms had no additional effect and were not more positively evaluated than generic information, they were not subjected to the next phase of efficacy evaluation.

\section{Efficacy of web-based computer-tailored nutrition information}

Computer tailoring was chosen as a strategy to induce changes in awareness, Intentions and behaviour, because it is an excellent technique for providing personal feedback to larger groups of people. Furthermore, the effectiveness of printed tailored feedback had been established in earlier studies (e.g.. Brug et al., 1999; Chapter 2). Technology has now made more advanced interactive, computer-based. and Internet-based applications of computer talloring possible. These new modes may make it possible to deliver computer-tailored feedback to large groups of people for lower costs than print versions. However, the efficacy of these more advanced applications needs to be established first.

The test of the immediate effects of the computer-tailored intervention (Chapters 3 and 4) revealed some effects on awareness and intention to change, compared to generic nutrition information and to self-test forms. These effects were found for the total study population and for the risk groups, that are, respondents with unfavourable diets at baseline. Further analyses revealed that respondents of all educational levels (related to awareness especially respondents with a medium or lower level of education) profited from the tailored intervention and that the tailored information was rated as more personally relevant, individualised and new than the generic information. Furthermore, respondents in the tailored intervention group were more likely to state that they would use the programme again. The web-based intervention was also appreciated by respondents with a lower level of computer literacy (Chapter 3). Based on these immediate effects, it was concluded that the tailored intervention had immediate effects on awareness and intention to change, and was thus subjected to the next phase of evaluation. 
The next phase of evaluation comprised testing the short- (three week) and longer-term (six month) effects of the computer-tailored intervention on awareness, intention to change and actual behaviour change (Chapters 5 and 6). The evaluation of the short-term effects revealed a higher level of awareness of fat and vegetable intake, a greater intention to change fat, fruit and vegetable intakes and a marginally higher vegetable intake, compared to generic web-based nutrition information and no information. Mean fruit intake was higher in the tailored intervention group among the 'at risk' subgroup and among respondents who were not aware of their low fruit intake at baseline ('unaware'). The tailored intervention was perceived as more individualised, personally relevant, interesting and new than the generic information. The evaluation of the longer-term effects also demonstrated group differences in favour of the tailored intervention for awareness, intention and intake of vegetables, and for intention to change fat intake. However, level of awareness had declined and intentions to change were no longer positive. Therefore, the findings of this phase of efficacy evaluation revealed that the computer-tailored intervention was effective in increasing awareness and intentions in the short-term. Effects on behaviour were limited to small effects for vegetable intake, and for fruit intake among the 'at risk' and 'unaware' subgroups. Perceived personal relevance, individualisation and interestingness of the information were identified as mediators for some of the effects of the computer-tailored intervention.

\section{Social comparison information}

In previous research, an optimistic bias in the comparison of one's own intake of fat, fruit and vegetables to that of others, was found to be associated with lack of awareness (see Chapter 1). However, little is known about the occurrence and nature of such interpersonal or social comparisons regarding dietary intake. An exploratory study was conducted to gain more insight into these issues, related to dietary fat intake. The study showed that many respondents could give examples of situations in which they compared themselves with others, whom they compare themselves with, and what aspects they observe in others. Comparisons were mainly with close others such as partners, relatives and friends. Respondents who intended to change, and younger respondents, were more likely to compare themselves with others. The results of this study gave preliminary insight in interpersonal comparisons related to dietary fat intake.

\section{Efficacy of positive and negative role model information}

Role model stories are a means of providing social comparison information in interventions. According to theory. positive and negative role model information could both have an effect on motivation and behaviour. Positive role model stories provide information about a person who performs better than the observer on a certain aspect, whereas negative role model stories provide information about someone who performs worse than the observer. To explore the potential effects 
of positive and negative role model information on awareness, intention to change and behaviour, a study was conducted in which the effects of such role model stories were examined in relation to fruit intake (Chapter 8 ). The test for immediate and short-term effects showed no effects for the positive or the negative role model stories on any outcome measures.

\section{Conclusion}

Of the interventions studied, the computer-tailored intervention was efficacious in increasing awareness and intention to change. Thus, this intervention was capable of inducing important progress toward behaviour change. Behaviour change was only induced marginally by the tallored intervention. Perceived personal relevance and individualisation of the information were found to be factors of importance to the effects of the tallored information.

The two studies related to social comparison information were a first attempt to study social comparison processes related to dietary intake. Their findings are limited and more research is needed. More insight in the social comparison processes related to (awareness of) dietary intake, would contribute to a better understanding of dietary behaviour and its determinants, and may provide suggestions for interventions that aim to modify these determinants.

\section{Methodological issues}

The conclusions should be evaluated in light of the methodological choices made and possible methodological limitations. The major methodological issues related to the studies presented in this thesis are the study design, measurement, sampiing and drop out and implementation and exposure to the intervention.

\section{Design}

All evaluation studies presented in this thesis were conducted as randomised triais in more or less controlled settings. Using experimental study designs allows ascribing observed effects to the intervention with the greatest level of certainty (Windsor, Baranowski, Clark, \& Cutter, 1994). Experimental designs are recommended for the evaluation of interventions. especially in the early phases of development (M. Campbell et al., 2000; Rossi et al., 1999). Through randomisation. equal study groups should be created, which rules out several threats to internal validity. Although strong evaluation designs were used in the studies presented, drop out and unsuccessful randomisation may have threatened internal validity in some of the studies. Furthermore, exposure to the assigned interventions could not be controlled in the studies evaluating the short- and long-term efficacy of the tailored intervention. 


\section{Measurement}

The first measurement issue relates to self-reported data. Self-reported data are sometimes referred to as 'soft' data, since they may be subject to socially desirable and inaccurate responses, which may lead to systematic bias in the information obtained. Most people are aware of the necessity of consuming a diet low in fat and containing plenty of fruit and vegetables, which may contribute to answering dietary questions in a 'correct' manner. On the other hand, participants may not know or remember exactly the frequency and amount of food items they consumed in the past month. Hence, there may be an under-reporting of fat intake and an over-reporting of fruit and vegetable intake. Using biomarkers as an indicator of dietary intake, like serum cholesterol levels for saturated fat intake, or vitamin $\mathrm{C}$ or carotenoids for fruit and vegetable intake, could help to overcome bias due to inaccurate self-reports and socially desirable responses. The logistic complexity, higher costs, and higher burden on participants that may lead to lower participation rates may, however, not outweigh the possible gains of obtaining more accurate information through biomarker evaluation, especially not in the earlier phases of intervention evaluation. For the psychosocial measures and subjective evaluation of interventions, it is not possible to use any means of data collection than self-reported data.

The choice of an appropriate measure and instrument for measuring a dietary outcome is an issue in all nutrition intervention studies (Contento, Randell, \& Basch, 2002). There is no gold standard for measuring dietary intake in large population groups. For each study, a trade-off between accuracy, validity and feasibility of a measurement tool has to be made. Physiological measures (i.e., biomarkers) may be the most objective or 'hard' measures for assessing dietary intakes, but the least feasible to use in research among larger groups. Multiple twenty-four hour recalls, or multi-day food diaries, may also have good validity, but are time consuming for respondents and therefore likely to result in low response rates. Moreover, such elaborate food consumption measurements may be an intervention in itself and may obscure the possible effects of the intervention tested. Food Frequency Questionnaires (FFGs) are generally short instruments that stil provide acceptable validity for assessing dietary intake. The feasibility of these FFgs, and their ability to detect changes over time makes them frequently used measurement tools in nutrition intervention evaluation studies. In the present study. FFgs that were validated among the Dutch adult population were used. The FFQ assessing fat intake was validated relative to 7 day diet records, which are accepted as a reliable and relatively valid instrument. Pearson correlation between the FFQ and the 7 day diet records were 0.71 and 0.69 for total fat and saturated fat respectively (Van Assema et al. 2001). This FFQ may be considered as sufficiently valid, since a correlation of 0.5 between the reference instrument and the FFQ is regarded as valid (Plesko, Cotugna, \& Aljadir, 2000). The Pearson correlation for test-retest reliability of a previous shorter version of this FFQ was .71 (Van Assema et al., 1992), and for the present version a Pearson correlation of .72 has been reported 
(Ronda, 2003). Spearman correlation between the fruit and vegetable questionnaire and 7 day diet records was .51 for fruit and .35 for vegetables (Van Assema et al. 2002). More recently, the fruit and vegetable questionnaire was validated relative to plasma concentrations of vitamin $\mathrm{C}$ and carotenoids. Spearman correlations were significant at .26 and .24 for vegetable intake and .37 and .39 for fruit intake related to vitamin $\mathrm{C}$ and carotenoids, respectively. Correlations of this magnitude are similar to those found in other biomarker studies. Spearman correlation for test-retest reliability ranged from .73 to .81 for vegetables and from .80 to .62 for fruits at one month and one year intervals (Bogers et al., accepted). The validity and reliability of both instruments may be considered acceptable, however not optimal. Both instruments are able to rank people according to fat fruit and vegetable intake. The instruments may, therefore, be limited in their sensitivity to detect small intervention effects.

In the studies presented in this thesis, self-rated intake and intention to change were the important intermediate outcome variables. Self-rated intake adjusted for actual intake was used as an indicator for awareness (Brug et al., 1998b). The intermediate variables were measured with single items. Single item assessments are associated with lower reliability and lower sensitivity, therefore changes may be harder to detect.

Recall bias refers to the fact that people with particular characteristics may be more able to recall certain events than others. Recall bias may particularly occur in retrospective assessments of events. The assessment of intake levels may be vulnerable to some recall bias, since dietary intake was assessed over the past month. Respondents who are more interested in diet and nutrition may, for example, have a better recall of their food consumption pattern. It is, however, expected that respondents with characteristics that could induce recall bias were equally distributed among the study groups. Recall bias may have affected the results found in the study on the occurrence and nature of social comparison related to dietary intake (Chapter 7). In this study respondents had to recall comparisons they made in the past. Respondents who intended to change may be more involved in the issue of dietary fat intake, and may therefore have been more able to recall events in which they had made comparisons regarding fat intake. If recall bias occurred, the greater comparison with others of respondents who intended to change may be overestimated, due to a better recall. In order to get a more accurate impression of the interpersonal comparisons related to dietary intake, instruments that overcome the shortcomings of retrospective methods in measuring social comparison should be used. Wheeler \& Miyake (1992), for example, suggested and tested such a method, a so-called event-contingent self-recording method. In this method social comparisons are recorded the moment they occur. Respondents are instructed to write down as exactly as possible whom they compared with, in what situation. related to what subject and so on, immediately after the comparison occurred. In this approach, respondents can also be asked to report how they felt before and after the comparison, and how they responded to the comparison in terms of thoughts or behaviour. 


\section{Recruitment of study participants and drop out}

The target group for the studies conducted in this thesis was the general Dutch population between 18 and 65 years of age. Many general approaches to recruit members of this target group, such as advertisements in newspapers, would probably attract people with specific interest in diet and nutrition, resulting in a selected population. With an approach in which people are invited personally, regardless of their interest in diet and nutrition, we attempted to decrease such selection. We therefore chose to recruit respondents through companies and education settings that would locate eligible respondents in the required age range, of various educational levels and occupation. This approach also provided the opportunity to create a controlled research setting on work-sites, which facilitated the conduct of the more controlled studies. Furthermore, by recruiting respondents through companies or education settings, the likelihood of people having access to a computer was high and it was anticipated that participation rates would increase if participants were allowed to participate during working hours. Work sites are potential places for implementing the interventions. Conducting the studies in these environments could provide some indication of feasibility for implementation. In spite of the personal approach to recruitment, participation rate was relatively low. The fact that in most of the studies presented respondents had more favourable dietary intakes than the general Dutch population indicates that our recruitment procedures also resulted in a selective study population.

In the studies examining the short- and longer-term efficacy of the computertailored intervention, there was a higher than expected drop out rate and we found evidence for a selective drop out according to study group. Respondents in the groups receiving nutrition education in some interactive form were more likely to drop out than those receiving no information. The effort required of participants to use the interactive programmes may have been a reason for the drop out. Interviews that were conducted among the drop outs in the tailored intervention group revealed that the most common reasons for drop out were lack of time, not having used the programme or forgetting to send in the questionnaire. Selection and drop out both limit the external validity. Drop out may also threaten internal validity. We attempted to overcome the selective drop out problem by conducting intentionto-treat analyses.

\section{Implementation success; exposure to the intervention}

Effective implementation and sufficient exposure to an intervention is of vital importance for an intervention to be effective. Only when an intervention was imple. mented as intended and respondents were exposed to it, can effects be ascribed to the intervention (Glasgow, 2002: Windsor et al., 1994). On the other hand, lack of effects of an intervention can sometimes be ascribed to insufficient implementation or exposure to the intervention. Concluding that an intervention was ineffective, when the case may have been that it was not implemented as intended or exposure 
was too low, would be a major error and is referred to as a type III error (Basch, Sliepcevich, Gold, Duncan, \& Kolbe, 1985). Therefore, monitoring of intervention implementation and exposure is of much importance. In the more controlled studies implementation of the intervention was according to plan and exposure to the intervention was under control. For the intervention to be effective, respondents had to read most of the information that was provided. The amount of the information read was measured using self-reports. Even though these self-reports give some indication of the use of the intervention, more advanced ways of measuring exposure and attention to specific parts of the information is feasible in laboratory settings. Technology makes it possible to more objectively assess the extent to which information is read, which parts are read in particular and how the brain is activated when reading the information, by means of tracking eyeball movements. electro encefalograms (EEG) or even functional magnetic resonance imaging (fMRI) (Kessels, Ruiter, \& Brug, submitted; Smidts, 2002). These techniques may provide more information on exposure related to effects, cognitive and affective processing of information and possible dose-response relationships.

In the field experiment described in the studies in Chapters Five and Six, the distribution of the intervention and instructions how to use it was according to plan, but actual use and exposure to the intervention was beyond control. Use of the intervention was assessed by means of a self-reported process evaluation questionnaire. Of the respondents who completed this questionnaire $84 \%$ reported having used the programme at least once. Due to drop out, not all respondents that were supposed to use the programme actually used it. Thus, actual rate of exposure to the intervention was $60 \%$. This indicates that exposure to the intervention may have been less than optimal. Furthermore, exposure to the different elements of the tallored intervention may have been insufficient. The tailored feedback information started with personal feedback on intake, which was specifically meant to raise awareness. The fact that effects on awareness and intention to change were found. may indicate that most respondents were af least exposed to these first parts of the feedback. On the other hand, the lack of an effect on behaviour may be caused by lack of exposure to the further information that was aimed at inducing behaviour change, by increasing self-efficacy and suggesting how to change.

Taking the approach of delivering the computer-tailored intervention either on a CD-ROM or through the worksite Intranet had the advantage that people who did not have access to the Internet could also participate in the study. However. it limited the opportunities of monitoring the proceeding through the programme. exposure to the different parts of the programme, and length of time spent on each page. Providing the intervention via a web-server on the Internet, would have offered the opportunity to trace how respondents proceeded through the programme. which parts of the intervention were completed, the amount of time spent on each page, and the frequency of visits to the site. 
To detect changes in determinants or behaviour, it is important to conduct posttest measurements at intervals allowing enough time for changes in (intermediate) outcomes to occur and to persist. The studies presented in this thesis comprised three time intervals for post-test measurement: immediately after exposure, three weeks post-intervention and six-months post intervention. Testing immediate effects of an intervention may be seen as a form of pilot testing. which provides more information about potential efficacy than qualitative pilot-testing. The immediate effects of the tailored intervention gave reason for a more extensive effect evaluation. A three-week follow-up was expected to be long enough to detect early changes in behaviour. Although at three weeks the effects of the tailored intervention on determinants were also found, only limited effects on behaviour were detected. This indicates that the intervention may not have been capable of producing behaviour change. It may, however, also be the case that the interval between exposure and follow-up was still too short. Especially when people did not use the programme soon after they had received it, there was not much time to induce behaviour change. The lack of an effect on dietary intake six months post-intervention does, however, indicate that the intervention did fail to produce substantial behaviour change.

\section{The interventions}

The quality and contents of interventions and the mode of delivery may be of influence to the effects of an intervention. In this section potential intervention-related barriers to efficacy of the feedback interventions will be discussed, and suggestions to improve the interventions are given. This section starts with comparing the two feedback interventions and than proceeds to a discussion of the delivery mode of the tailored intervention, responses to feedback and the step from intention to behaviour.

\section{Feedback interventions}

The feedback provided in the tallored intervention was much more refined than that in the self-test forms and the tailored information was written in such a way that the recipient was personally addressed. The level of specificity of the feedback information provided through the computer-tailored information seemed to be sufficient to increase awareness and intention to change. The results made it apparent that the feedback provided in the self-test forms was not sufficient to increase awareness and intention to change. This may be due to the feedback being too brief and general, or because the self-test forms were not perceived as individualised. which was found to be of importance for the effects of the tallored intervention. Increasing the level of specificity and personalisation of the self-test forms may 
increase the efficacy of such interventions.

The web-based computer-taflored intervention produced effects on awareness and intention to change, but effects on behaviour were limited and not evident for fat intake. This is in contrast with the results of studies evaluating the efficacy of print delivered computer-tailored interventions (e.g., Brug et al., 1999). The content of the web-based tailored intervention was an adaptation and extension of an effective print-delivered tailored information system. Since the content of the webbased intervention was similar to that of the print version, the absence of effects on behaviour may be due to the interactive channel through which the information was provided.

Another issue concerning computer tafloring relates to the provision of feedback. Providing personal and individualised feedback is the core element of computer tafloring. There are, however, many ways in which people can respond to feedback (Kluger \& DeNisi, 1996). More insight in these responses to feedback in terms of subsequent behaviour may provide the opportunity to improve computer-tailored interventions. Finally, since the tailored intervention did not result in effects on behaviour, stronger strategies to induce the step from intention to behaviour may be required, than those used in the web-based computer-tailored intervention.

\section{The channel of delivery of the tailored information}

The Internet and especially the WWW is highly appreciated as a way to provide health education information. The potential high reach, instant availability, costeffectiveness, and the possibility to include interactive, interpersonal and video and audio elements to make the information presentation more attractive, are seen as potential advantages of the medium. Because of these advantages, the WWW is considered to be a suitable channel to provide computer-tailored information (Brug et al.. 2003). Although the Internet seems to hold great promise for (computer-tailored) health education. little is known about the potential efficacy of webdelivered behaviour-change interventions. Therefore, it is not surprising that the importance of evidence based Internet interventions has been advocated repeatedly (Eng, Gustafson, Henderson, Jimison, \& Patrick, 1999; Robinson, Patrick, Eng, \& Gustafson, 1998).

The studies described in this thesis are among the first to formally evaluate a web-based computer-tailored nutrition intervention. The results of the studies presented may be a reason for a slightly more reserved view about the health education potential of the WWW, since it may also incorporate some important barriers to effectively communicate behaviour change information.

First, the Internet may improve possibilities to distribute health information. but it may reduce exposure to the content of the message. The Internet may be a 'fleeting' medium, used to quickly find and scan information and then move on to other pages. Therefore, people may read and process information provided through the WWW more shallowly. Further, reading information from a computer screen is still not as convenient as reading print information for many people, a factor 
which may hinder careful reading and processing of the information (Murphy et al., 2003).

Second, the WWW and other interactive media enable provision of feedback immediately after completion of an assessment. This immediate feedback is likely to increase the accuracy of the tailored information. since it is more likely that the feedback indeed matches the recipient's behaviour and cognitions at that moment. However, providing feedback immediately may reduce the credibility and the authority of the feedback as well. Recipients may not 'trust' personal feedback that is provided instantly after completion of an assessment.

Third, the Internet enables provision of repeated, ipsative feedback for relatively low costs. Some evidence points in the direction that the effect of interactive interventions using the Internet or other computer based information delivery systems may be dependent on repeated feedback sessions (Anderson et al., 2001; Baranowski, Baranowski, Cullen, Marsh, Islam, et al. 2003). However, attracting people repeatedly to the same web-site and keeping them long enough to obtain and read all relevant feedback information is an additional challenge.

Fourth, although the Internet is appreciated as a channel for tailored health information, this does not necessarily mean that people will use computer-tailored web-based health education. Actual use means starting the computer, logging in on the Internet, searching for the right web-site, opening the appropriate programme, finding out how to use the programme, etc. As was mentioned before. the relatively high drop out rate in the groups that received the intervention in the studies presented in this thesis may indicate that using the intervention was indeed too much of an effort. Other evaluation studies of interventions provided through the Internet also showed low actual usage, and diminished usage over time (Napolitano. Fotheringham. Tate. Sciamanna, Leslie, et al. 2003; Ritterband, Gonder-Frederick, Cox, Clifton, West, \& Borowitz, 2003).

Fifth, the way in which information through interactive media is ideally provided, is through brief and to-the-point information segments, backed up with interactive video and audio elements. It is, however, not known if such brief information segments provide enough information to be persuasive. In the present study, we 'translated' an existing feedback library file from a print delivered taflored information system to an interactive web-based intervention. This procedure may have resulted in insufficient utilisation of potential effect inducing features of interactive programmes, i.e., the text segments may, for example, have been too long, with too little interactivity. More insight into the optimal length of information segments. amount of interactivity, use of colours, audio and video elements may help to further improve web-based interactive computer tailoring.

\section{Feedback as a method for increasing awareness}

Personal and normative feedback was provided as a method for increasing awareness of personal risk behaviour and personal intake levels were compared with the recommended intake level. The expected working mechanism of feedback would 
be that through the comparison of personal intake with a standard, people would become more aware of the possible inadequacy of their intake levels, which would result in a higher motivation to improve intake. The feedback provided through the tailored intervention indeed seems to have resulted in a higher level of awareness and greater intention to change. These findings are encouraging. However, little is known about how exactly the feedback resulted in these effects. Feedback is a complex strategy to change and widely used in health education interventions. The working mechanism of feedback in the process of behaviour change is not very well understood (DiClemente et al. 2001). Effects of feedback interventions on performance have found to be quite variable (Kluger \& DeNisi, 1996) and to be dependent on factors such as the nature of the behaviour that needs to be improved. whether or not performance goals were set, the perceived importance and commitment to this goal, the appraisal of the feedback, self-efficacy, capabilities to improve the behaviour, the number of feedback sessions, whether or not the 'self is threatened by the feedback, whether the feedback relates to objective standards or to social information, and so on (Carver \& Scheier, 1998; Klein. \& Weinstein, 1997: Klein. 2003: Locke \& Latham, 1990). More insight into how personalised feedback affects cognition, motivation and behaviour may provide the opportunity to improve efficacy of computer-tailored feedback interventions.

\section{The gap between intention and behaviour}

The computer-tailored intervention evaluated in the studies presented was largely unsuccessful in helping people to bring their intention into action. As mentioned earlier, this may have resulted from insufficient exposure to or insufficient processing of the information provided by the intervention. It may, however, also be the case that the intervention did not include a strong enough strategy to overcome the 'intention - behaviour gap', since the intervention primarily aimed at overcoming the lack of awareness barrier. Most behavioural determinant models are motivational models and do predict the intention to perform a particular behaviour rather well. Even though intention is considered to be the most proximal determinant to behaviour, an intention to engage in a behaviour does not always result in performance of that behaviour (e.g., Norman \& Conner, 1995: Sheeran, 2002). Gollwitzer, among others, argued that effective goal pursuit consists of two phases, a motivational phase and a volitional phase (Gollwitzer. 1999). Whereas the motivational phase is concerned with thinking about and deciding to achieve a particular goal, the volitional phase consists of planning actions in order to achieve that goal. Gollwitzer's central concept in this volitional phase is that of implementation intentions, i.e., specific plans about how, when and where to act in order to achieve the behavioural goal or enact the behavioural intention (Gollwitzer, 1999; Gollwitzer \& Brandstātter, 1997). Suggestions to make implementation intentions were included in the tailored interventions about fruit and vegetables, but our study design did not allow us to evaluate the effects of the implementation intentions specifically. More research is needed to understand how this strategy can be incorporated ef- 
fectively in computer-tailored interventions in order to enhance efficacy.

There may also be other strategies to induce the step from intention to behaviour that can be incorporated in computer-tailored interventions. Dietary behaviour is a complex behaviour. consisting of several sub-behaviours, that can be performed and modified in hundreds of different ways and that have to be repeated day after day. It is, therefore, not possible to prescribe exactly how an individual has to change. It requires self-management skills to consume a healthy diet day after day. Including instructions for self-management strategies in the tailored information could be another possibility to improve the effects on behaviour change of the computer-tailored intervention tested.

\section{Implications}

\section{Implications for nutrition education practice}

The web-based tailored intervention was efficacious in increasing awareness and intention to change fat, fruit and vegetable intakes. This intervention was also evaluated most positively by the respondents, including users of lower education levels and with low computer literacy. This intervention is now ready to be used on a larger scale for the purpose of increasing awareness and intention to change. preferably combined with methods that induce the step from intention to behaviour.

The Internet is a challenging and attractive medium for delivering nutrition information, but it is not a panacea and has its limitations. Use of this medium should be carefully considered and effects of interventions provided through it should be evaluated for their effects, even when it was an effective intervention in print form. As the studies presented in this thesis highlight, the effect of the information provided through an interactive channel may not be the same as that of equivalent information provided through traditional print media.

Efficacy evaluation of interventions in early phases of development is important. Even though a pilot-study into immediate effects may seem costly and time consuming, it provides an indication for potential effects. It provides richer information than mere qualitative pilot testing. Small-scale efficacy evaluation is expected to be cost-effective in the long run, since only interventions that have the potential to be effective will be implemented on a large scale. For that purpose it is necessary that efficacy evaluation occurs before large-scale implementation is initiated rather than afterwards " as was the case with the self-test forms.

\section{Suggestions for further research}

The studies presented in this thesis were among the first to explore the effects of a more advanced application of a computer-tailored nutrition intervention. More research into the effects of tailored interventions provided through interactive media is necessary. to compile evidence for the efficacy of computer-tailored be- 
haviour change interventions provided through the Internet or other interactive media. In addition to this, research is needed into how people use and process information provided through the Internet or other 'modern' interactive media and how information through this media can best be presented in order to be persuasive. Furthermore, the apparent discrepancy in effects between studies using print computer-tailored feedback and computer-tailored feedback provided through interactive media warrants a systematic comparison between both delivery modes in a randomised controlled trial.

Feedback is the core element of the computer-tailoring technique. More insight into how feedback facilitates (or hinders) motivation and subsequent behaviour would assist in improving the efficacy of computer-tailored behaviour change interventions. Computer-tailored interventions can further be improved by including strategies that more strongly induce the step from motivation to behaviour. Research is needed on how such strategies can optimally and effectively be included in interventions.

The study presented in Chapter 5 contributes to the still small body of evidence on how computer-tailored interventions exert their effects. More insight in these working mechanisms is needed to facilitate improvement of computer-tailored interventions.

The research into social comparison processes presented in this thesis is preliminary. To more fully appreciate the potential value of social comparison processes in understanding or changing dietary behaviour, further research is warranted. Research aimed at understanding the processes of social comparison related to dietary intake, should use methods that are less dependent on retrospective interviewing. Such an approach could also provide more insight into how social comparison affects subsequent behaviour. 


\section{References}

Abrams, D.B., Emmons, K.M., \& Linnan, L.A. (1997). Health behavior and health education: The past, present, and future. In K. Glanz, F.M. Lewis, \& B.K. Rimer (Eds.). Health behaviour and health education: Theory, research, and practice (pp. 453-478). San Francisco: Jossey-Bass.

Abrams, D.B., Mills, S., \& Bulger, D. (1999). Challenges and future directions for taklored communication research. Annals of Behavioral Medicine, 21, 299-306.

Ajzen, I. (1988). Attitudes, personality and behavior. Milton Keynes: Open University Press.

Anderson, E.S., Winett, R.A., Wojcik, J.R., Winett, S.G., \& Bowden. T. (2001). A computerized social cognitive intervention for nutrition behavior: Direct and mediated effects on fat, fiber, fruits and vegetables, self-efficacy, and outcome expectations among food shoppers. Annals of Behavioral Medicine, 23, 88-100.

Armitage, C.J.. \& Conner. M. (2000). Social cognition models and health behaviour: A structured review. Psychology and Health, 15, 173-189.

Bandura, A. (1986). Social foundations of thought and action. Englewood Cliffs: PrenticeHall.

Bandura, A. (1997). Self-efficacy: The exercise of control. New York: W.H. Freeman and Company.

Baranowski, T., Baranowski, J., Cullen, K.W., Marsh. T., Islam. N., Zakeri, I., HonessMorreale, L., \& deMoor, C. (2003). Squire's Quest! Dietary outcome evaluation of a multimedia game. American Journal of Preventive Medicine, 24, 52-61.

Baranowski, T., Cullen, K.W., \& Baranowski, J. (1999). Psychosocial correlates of dietary intake: Advancing dietary intervention. Annual Review of Nutrition, 19, 17-40.

Baron, R.M., \& Kenny, D.A. (1986). The moderator-mediator variable distinction in social psychological research: Conceptual, strategic, and statistical considerations. Journal of Personality and Social Psychology, 51, 1173-1182.

Bartholomew, L.K., Parcel, G.S., Kok, G., \& Gottlieb, N.H. (2001). Intervention Mapping: Designing theory- and evidence-based health promotion programs. Mountain View: Mayfield.

Basch. C.E., Sliepcevich, E.M., Gold, R.S., Duncan, D.F., \& Kolbe, L.J. (1985). Avoiding. 
type III errors in health education program evaluations: A case study. Health Education Guarterly, 12, 315-331.

Beer-Borst, S., Hercberg. S., Morabia, A., Bernstein, M.S., Galan, P., Galasso, R., Giampaoll, S., McCrum, E., Panico, S., Preziosi, P., Ribas. L., Serra-Majem, L., Vescio, M.F., Vitek, O., Yarnell, J., \& Northridge, M.E. (2000). Dietary patterns in six European populations: Results from EURALIM. a collaborative European data harmonization and information campaign. European Journal of Clinical Nutrition. 54, 253-262.

Blalock, S.J., DeVellis, B.M., Patterson, C.C., Campell, M.K., Orenstein, D.R., \& Dooley, M.A. (2002). Effects of an osteoporosis prevention program incorporating taflored educational materials. American Journal of Health Promotion, 16. 146-156.

Block, G., Miller, M., Harnack, L., Kayman, S., Mandel, S., \& Cristofar, S. (2000). An interactive CD-ROM for nutrition screening and counseling. American Journal of Public Health, 90, 781-785.

Bock, B.C., Marcus, B.H., Pinto, B.M., \& Forsyth. L.H. (2001). Maintenance of physical activity following an individualized motivationally tailored intervention. Annals of Behavioral Medicine, 23, 79-87.

Boer, H., \& Seydel, E.R. (1995). Protection motivation theory. In M. Conner, \& P. Norman (Eds.), Predicting health behaviour: Research and practice with social cognition models (pp. 95-120). Buckingham: Open University Press.

Bogers, R.P., Van Assema, P., Kester, A.D.M., Westerterp, K.R., \& Dagnelie, P.C. (Accepted). Reproducibility, validity and responsiveness to change of a short questionnaire for measuring intake of fruits and vegetables. American Journal of Epidemiology.

Bowen, D.J.. Fries, E., \& Hopp. H.P. (1994). Effects of dietary fat feedback on behavioral and psychosocial variables. Journal of Behavioral Medicine. 17, 589-604.

Brug. J. (1999). Dutch research into the development and impact of computer-tailored nutrition education. European Journal of Clinical Nutrition, 53, S78-S82.

Brug. J.. Campbell. M.. \& Van Assema. P. (1999). The application and impact of computer-generated personalized nutrition education: A review of the literature. Patient Education and Counseling. 36, 145-156.

Brug. J., \& De Vries, H. (1999). Computer-tailored education. Patient Education and Counseling, 36.

Brug. J., Glanz, K., Van Assema, P., Kok, G., \& Van Breukelen, G.J.P. (1998a). The impact of computer-tallored feedback and iterative feedback on fat, fruit, and vegetable intake. Health Education and Behavior, 25, 517-531.

Brug. J., Hospers. H.J., \& Kok, G. (1997). Differences in psychosocial factors and fat consumption between stages of change for fat reduction. Psychology and Health, 12. 719-727.

Brug. J., \& Kok, G.J. (1995). Misconceptie van consumenten over eigen vetconsumptie [Consumers' misconception about their fat consumption]. Voeding. 56, 11-14.

Brug. J.. \& Lechner, L. (1995). Nederlanders overschatten hun groente- en fruitconsumptie [The Dutch over-estimate their vegetable and fruit consumption]. Voeding. 56. 20-23.

Brug, J., Lechner, L., \& De Vries, H. (1995). Psychosocial determinants of fruit and vegetable consumption. Appetite, 25, 285-296.

Brug, J., Oenema. A., \& Campbell, M. (2003). Past, present and future of computer-tailored nutrition education. American Journal of Clinical Nutrition, 77. 1028S-1034S.

Brug. J., Steenhuis, I., Van Assema, P., \& De Vries, H. (1996). The impact of a computertailored nutrition intervention. Preventive Medicine, 25, 236-242. 
Brug, J., Van Assema, P., \& Kok. G. (1998b). Misconceptie van consumptie van vet. groente en fruit: Oorzaken en implicaties voor voedingsvoorlichting [Misconception of fat, vegetable and fruit consumption: Causes and implications for nutrition education]. Tijdschrift voor Gezondheidswetenschappen, 76, 17-23.

Brug. J.. \& Van Assema, P. (2000). Differences in use and impact of computer-tailored dietary fat-feedback according to stages of change and education. Appetite, 34, 285293.

Brug, J., \& Van Assema, P. (2001). Beliefs about fat: Why do we hold beliefs about fat and why and how do we study these beliefs? In L.J, Frewer, E. Risvik, \& H. Schifferstein (Eds.), Food, people and society: A European perspective of consumers' food choices (pp. 39-54). Berlin: Springer-Verlag.

Brug, J., Van Assema, P., Kok, G., Lenderink, T., \& Glanz, K. (1994). Self-rated dietary fat intake: Association with objective assessment of fat, psychosocial factors, and intention to change. Journal of Nutrition Education, 26. 218-223.

Bull, F.C., Kreuter, M.W., \& Scharff, D.P. (1999). Effects of tailored, personalized and general health messages on physical activity. Patient Education and Counseling. 36 , 181-192.

Buunk, B.P., \& Gibbons, F.X. (Eds.). (1997). Health, coping, and well-being: Perspectives from social comparison theory. Mahwah: Lawrence Erlbaum Associates.

Buunk, B.P., Ybema, J.F., Gibbons, F.X., \& Ipenburg. M. (2001). The affective consequences of social comparison as related to professional burnout and social comparison orientation. European Journal of Social Psychology, 31, 337-351.

Campbell, M., Fitzpatrick, R., Haines, A., Kinmonth, A.L., Sandercock, P., Spiegelhalter, D., \& Tyrer, P. (2000). Framework for design and evaluation of complex interventions to improve health. BMJ, 321, 694-696.

Campbell, M.K., DeVellis, B.M., Strecher, V.J., Ammerman, A.S., DeVellis, R.F., \& Sandler, R.S. (1994). Improving dietary behavior: The effectiveness of tallored messages in primary care settings. American Journal of Public Health, 84, 783-787.

Campbell, M.K., Honess-Morreale, L., Farrell, D., Carbone, E.. \& Brasure, M. (1999). A tailored multimedia nutrition education pilot program for low-income women recelving food assistance. Health Education Research, 14. 257-267.

Campbell, M.K.. Tessaro, I., DeVellis, B., Benedict, S., Kelsey, K., Belton, L., \& Henriquez-Roldan. C. (2000). Tailoring and targeting a worksite health promotion program to address multiple health behaviors among blue-collar women. American Journal of Health Promotion, 14. 306-313.

Campbell, M.K., Tessaro, I., DeVellis, B., Benedict, S., Kelsey, K., Belton, L., \& Sanhueza, A. (2002). Effects of a tailored health promotion program for female blue-collar workers: Health works for women. Preventive Medicine, 34, 313-323.

Carver, C.S.. \& Scheier, M.F. (1998). On the self-regulation of behavior. Cambridge: Cambridge University Press.

Celio, A.A., Winzelberg, A.J., Wilfley, D.E., Eppstein-Herald, D., Springer, E.A., Dev, P., \& Taylor, C.B. (2000). Reducing risk factors for eating disorders: Comparison of an Internet and a classroom-delivered psychoeducational program. Journal of Consulting and Clinical Psychology. 68, 650-657.

Centraal Begeleidingsorgaan voor de Intercollegiale Toetsing (CBO), in samenwerking met de Nederlandse Hartstichting. et al. (1998). Behandeling en preventie van coronaire hartziekten door verlaging van de plasmacholesterol-concentratie /Treatment and prevention of coronary heart diseases, by lowering plasma concentrations of cho- 
lesterol]. Utrecht: Centraal Begeleidingsorgaan voor de Intercollegiale Toetsing.

Centraal Bureau voor de Statistiek (CBS). (2004). Statline. ICT en media gebruik 19982002 [IT and use of media 1998-2002]. Avallable from: URL: http://statline.cbs.nl/ StatWeb. Accessed March 5, 2004.

Cline, R.J.W., \& Haynes, K.M. (2001). Consumer health information seeking on the Internet: The state of the art. Health Education Research, 16, 671-692.

Conner, M., \& Armitage. C.J. (1998). The theory of planned behavior: A review and avenues for further research. Journal of Applied Social Psychology. 28, 1429-1464.

Conner, M., \& Norman, P. (1995). The role of social cognition in health behaviours. In M. Conner, \& P. Norman (Eds.). Predicting health behaviour: Research and practice with social cognition models (pp. 1-22). Buckingham: Open University Press.

Contento, I., Balch, G.I., Bronner, Y.L., Lytle, L.A., Maloney, S.K., Olson. C.M.. \& Swadener, S.S. (1995). The effectiveness of nutrition education and implications for nutrition education policy, programs and research: A review of research. Journal of Nutrition Education, 27, 277-422.

Contento, I.R., Randell, J.S., \& Basch. C.E. (2002). Review analysis of evaluation meas ures used in nutrition education intervention research. Journal of Nutrition Education and Behavior, 34, 2-25.

Davis, R.N. (1999). Web-based administration of a personality questionnaire: Comparison with traditional methods. Behavior Research Methods, Instruments and Computers, 31, 572-577.

De Bourdeaudhuij. 1., \& Brug. J. (2000). Tailoring dietary feedback to reduce fat intake: An intervention at the family level. Health Education Research, 15, 449-462.

De Bourdeaudhuij. I., Brug. J., Vandelanotte, C., \& Van Oost, P. (2002). Differences in impact between a family- versus an individual-based tallored intervention to reduce fat intake. Health Education Research. 17. 435-449.

De Nooljer, J., Lechner, L.. Candel, M., \& De V'ries, H. (2002). A randomized controlled study of short-term and long-term effects of tailored information versus general information on intention and behavior related to early detection of cancer. Cancer Epidemiology. Biomarkers and Prevention. 11, 1489-1491.

De Nooijer, J., Lechner, L., \& De Vries, H. (2002). Tailored versus general information on early detection of cancer: A comparison of the reactions of Dutch adults and the impact on attitudes and behaviors. Health Education Research. 17. 239-252.

De Vries, H., \& Brug. J. (1999). Computer-tailored interventions motivating people to adopt health promoting behaviours: Introduction to a new approach. Patient Education and Counseling. 36, 99-105.

De Vries, H., Dijkstra. M.. \& Kuhlman, P. (1988). Self-efficacy: The third factor besides attitude and subjective norm as a predictor of behavior intention. Health Education Research, 3. 273-282.

De Vries, H., \& Mudde, A.N. (1998). Predicting stage transitions for smoking cessation applying the attitude-social influence-efficacy model. Psychology and Health. 13. 369-385.

Delichatsios. H.K., Friedman, R.H., Glanz, K., Tennstedt, S., Smigelski, C., Pinto, B.M., Kelley, H., \& Gillman, M.W. (2001). Randomized trial of a 'talking computer' to improve adults' eating habits. American Journal of Health Promotion, 15. 215-224.

DiClemente, C.C., Marinilli. A.S., Singh. M.. \& Bellino. L.E. (2001). The role of feedback in the process of health behavior change. American Journal of Health Behavior. 25. 217-227. 
Dijkstra, A., \& De Vries, H. (1999). The development of computer-generated tailored interventions. Patient Education and Counseling, 36, 193-203.

Dijkstra, A., De Vries, H., \& Roijackers, J. (1998). Long-term effectiveness of computergenerated tailored feedback in smoking cessation. Health Education Research, 13, 207-214.

Dijkstra, A., De Vries, H., \& Roijackers, J. (1999). Targeting smokers with low readiness to change with tailored and nontailored self-help materials. Preventive Medicine, 28. 203-211.

Eng, T.R., Maxfield, A., Patrick, K., Deering, M.J., Ratzan, S.C.. \& Gustafson, D.H. (1998). Access to health information and support, a public highway or a private road? JAMA, 280, 1371-1375.

Eng, T.R., Gustafson, D.H., Henderson, J., Jimison. H., \& Patrick, K. (1999). Introduction to evaluation of interactive health communication applications. American Journal of Preventive Medicine, 16, 10-15.

Ezzati, M., Lopez, A.D., Rodgers, A., Vander Hoorn, S., Murray, C.J.L. \& Comparative Risk Assessment Collaborating Group. (2002). Selected major risk factors and global and regional burden of disease. The Lancet, 360, 1347-1360.

Festinger, L. (1954). A theory of social comparison processes. Human Relations, 7, 117 140.

Fishbein, M., \& Ajzen, I. (1975). Belief, attitude, intention and behavior: An introduction to theory and research. Reading: Addison-Wesley.

Flay, B.R., \& Petriatis, J. (1994). The theory of triadic influence: A new theory of health behavior with implications for preventive interventions. Advances in Medical Sociology, 4, 19-44.

Fotheringham, M.J., Owies, D., Leslie, E., \& Owen, N. (2000). Interactive health communication in preventive medicine: Internet-based strategies in teaching and research. American Journal of Preventive Medicine, 19, 113-120.

Ganji, V., \& Betts, N. (1995). Fat, cholesterol, fiber and sodium intakes of US population: Evaluation of diets reported in 1987-88 nationwide food consumption survey. European Journal of Clinical Nutrition, 49, 915-920.

Gibbons, F.X., \& Buunk, B.P. (1999). Individual differences in social comparison: Development of a scale of social comparison orientation. Journal of Personality and Social Psychology, 76, 129-142.

Glanz, K., Basil, M., Maibach, E., Goldberg, J., \& Snyder, D. (1998). Why Americans eat what they do: Taste, nutrition, cost, convenience, and weight control concerns as influences on food consumption. Journal of the American Dietetic Association, 98. 1118-1126.

Glanz, K., Brug, J., \& Van Assema, P. (1997). Are awareness of dietary fat intake and actual fat consumption associated? A Dutch-American comparison. European Journal of Clinical' Nutrition, 51, 542-547.

Glasgow, R.E. (2002). Evaluation of theory-based interventions: The RE-AIM model. In K. Glanz, B.K. Rimer, \& F.M. Lewis (Eds.). Health behavior and health education: Theory research and practice (pp. 530-544). San Francisco: Jossey-Bass.

Gollwitzer. P.M. (1999). Implementation intentions: Strong effects of simple plans. American Psychologist, 54, 493-503.

Gollwitzer, P.M.. \& Brandstātter. V. (1997). Implementation intentions and effective goal pursuit. Journal of Personality and Social Psychology. 73, 186-199.

Green, L.W., \& Kreuter, M.W. (1999). Health promotion planning: An educational and 
ecological approach. Mountain View: Mayfield.

Health Council of the Netherlands. (2001). Dietary Reference Intakes: Energy. proteins, fats and digestible carbohydrates. (Publication no. 2001/19). The Hague: Health Councll of the Netherlands.

Health Councll of the Netherlands. (2003). Overweight and obesity. (Publication no. 2003/07). The Hague: Health Council of the Netherlands.

James, A.S., Campbell, M.K., \& Hudson, M.A. (2002). Perceived barriers and benefits to colon cancer sereening among African Americans in North Carolina: How does perception relate to screening behavior? Cancer Epidemiology. Biomarkers and Pre. vention, 11, 529-534.

Joshipura, K.J., Hu. F.B., Manson, J.E., Stampfer, M.J., Rimm, E.B., Speizer, F.E., Colditz, G., Ascherio, A., Rosner, B., Spiegelman. D.. \& Willet. W.C. (2001). The effect of fruit and vegetable intake on risk for coronary hearth disease. Annals of Internal Medicine, 134, 1106-1114.

Kearney, M., Gibney, M.J., Martinez, J.A., De Almeida, M.D.V., Friebe, D., Zunft, H.J.F.. Widhalm, K., \& Kearney, J.M. (1997). Perceived need to alter eating habits among representative samples of adults from all member states of the European Union. European Journal of Clinical Nutrition, 51, S30-S35.

Kessels, L., Ruiter, R., \& Brug. J. (submitted). De effecten van een voedingsadvies-opmaat op aandacht: Een ERP benadering TThe effects of computer-tailored nutrition education on attention: An ERP approach].

Key, T.J.. Allen, N.E.. Spencer, E.A., \& Travis, R.C. (2002). The effect of diet on risk of cancer. The Lancet. 360, 861-868.

Klein, W.M., \& Weinstein. N.D. (1997). Social comparison and unrealistic optimism about personal risk. In B.P. Buunk, \& F.X. Gibbons (Eds.). Health. coping and well being: Perspectives from social comparison theory (pp. 25-61). Mahwah: Lawrence Erlbaum Associates.

Klein, W.M.P. (2003). Effects of objective feedback and 'single other' or 'average other' social comparison feedback on performance judgments and helping behavior. Personality and Social Psychology Bulletin. 29. 418-429.

Kiuger, A.N., \& DeNisi, A. (1996). The effects of feedback interventions on performance: A hisiorical review, a meta-analysis, and a preliminary feedback intervention theory. Psychological Bulletin, 119, 254-284.

Koek, H.L., Van Leest, L.A.T.M., Verschuren, W.M.M., \& Bots, M.L. (2003). Hart-en vaatziekten in Nederland 2003, cüfers over leefstijl- en risicofactoren, ziekte en sterfte. [Cardiovascular diseases in the Netherlands 2003, figures on life-style and risk factors, disease and mortalityl. Den Haag: Nederlandse Hartstichting.

Kok, G.. Schaalma, H., \& Brug, J. (2000). Planmatige gezondheidsvoorlichting: een inleiding [Planned health education: an introduction]. In J. Brug. H. Schaalma, G. Kok, R.M. Meertens, \& H.T. Van der Molen (Eds.). Gezondheidsvoorlichting en gedragsverandering: Een planmatige aanpak (pp. 15-32). Assen: Van Gorcum.

Kok, G., Schaalma, H., De Vries, H., Parcel. G.. \& Paulussen. T. (1996). Social psychology and health education. European Review of Social Psychology. 7. 241-282.

Krebs-Smith, S.M., Heimendinger, J., Patterson, B.H., Subar, A.F., Kessler, R.K., \& Pivonka. E. (1995). Psychosocial factors associated with fruit and vegetable consumption. American Journal of Health Promotion, 10. 98-104.

Kreuter, M., Farrell, D., Olevitch, L.. \& Brennan, L. (2000). Tailoring health messages: Customizing communication with computer technology. Mahwah: Lawrence Erlbaum 
Associates.

Kreuter, M.W., Bull, F.C., Clark, E.M., \& Oswald, D.L. (1999). Understanding how people process health information: A comparison of tailored and non-tailored weightloss materials. Health Psychology, 18, 487-494.

Kreuter, M.W., Oswald, D.L. Bull, F.C.. \& Clark. E.M. (2000). Are tailored health education materials always more effective than non-tailored materials? Health Education Research, 15, 305-315.

Kreuter, M.W., \& Skinner, C.S. (2000). Tailoring: what's in a name. Health Education Research, 15, 1-4.

Kreuter, M.W., \& Strecher. V.J. (1996). Do tailored behavior change messages enhance the effectiveness of health risk appraisal? Results from a randomized trial. Health Education Research, 11, 97-105.

Lechner, L., Brug, J., \& De Vries, H. (1997). Misconceptions of fruit and vegetable consumption: Differences between objective and subjective estimation of intake. Journal of Nutrition Education, 29, 313-320.

Lechner, L., Brug. J., De Vries, H., Van Assema, P. \& Mudde, A. (1998). Stages of change for fruit, vegetable and fat intake: Consequences of misconception. Health Education Research, 13, 1-11.

Leonard, K.P., Bartholomew, L.K., Swank, P.R., \& Parcel, G.S. (1995). A comparison of two approaches to education about carrier testing for cystic fibrosis. Journal of Genetic Counseling, 4, 97-113.

Locke, E.A., \& Latham. G.P. (1990). A theory of goal setting and task performance. Englewood Cliffs: Prentice Hall.

Lockwood, P., Jordan, C.H., \& Kunda, Z. (2002). Motivation by positive or negative role models: Regulatory focus determines who will best inspire us. Journal of Personality. and Social Psychology. 83, 854-864.

Lutz, S.F., Ammerman, A.S., Atwood, J.R., Campbell, M.K., DeVellis, R.F., \& Rosamond, W.D. (1999). Innovative newsletter interventions improve fruit and vegetable consumption in healthy adults. Journal of the American Dietetic Association, 99, 705709.

McGuire, W.J. (1985). Attitudes and attitude change. In G. Lindzey, \& E. Aronson (Eds.). The handbook of social psychology (pp. 233-346). New York: Random House.

McKenzie, J.F., \& Smeltzer, J.L. (2001). Planning, implementing and evaluating health promotion programs: A primer. Boston: Allyn and Bacon.

Murphy, P.K., Long, J.F., Holleran, T.A., \& Esterly, E. (2003). Persuasion online or on paper: A new take on an old issue, Learning and Instruction, 13, 511-532.

Murray, C.J.L., \& Lopez, A.D. (1997). Global mortality, disability, and the contribution of risk factors: Global burden of disease study. The Lancet, 349, 1436-1442.

Napolitano, M.A., Fotheringham, M., Tate, D., Sciamanna, C., Leslie, E., Owen, N.. Bauman, A., \& Marcus, B. (2003). Evaluation of an Internet-based physical activity intervention: A preliminary investigation. Annals of Behavioral Medicine, 25, 92-99.

Ness, A.R. \& Powles, J.W. (1997). Fruit and vegetables, and cardiovascular disease: A review. International Journal of Epidemiology. 26, 1-13.

Norman, P., \& Conner, M. (1995). The role of social cognition models in predicting health behaviours: Future directions. In M. Conner, \& P. Norman (Eds.), Predicting health behaviour: Research and practice with social cognition models (pp. 197-225). Buckingham: Open University Press.

Oenema, A., \& Brug, J. (2003). Feedback strategies to raise awareness of personal di- 
etary intake: Results of a randomized controlled trial. Preventive Medicine, 36. 429. 439.

Oenema, A., Brug, J., \& Lechner L. (2001). Web-based tailored nutrition education: Results of a randomized controlled trial. Health Education Research, 16, 647-660.

Oenema. A., Tan, F., \& Brug. J. (Accepted). Short-term efficacy of a web-based computer-tallored nutrition intervention: main effects and mediators. Annals of Behavioral Medicine.

Orbell, S., \& Sheeran, P. (2002). Changing health behaviours: The role of implementation intentions. In D. Rutter, \& L. Guine (Eds.). Changing health behaviour: Intervention and research with social cognition models (pp. 123-137). Buckingham: Open University Press.

Petty, R.E.. Barden, J., \& Wheeler. S.C. (2002). The elaboration likelihood model of persuasion: Health promotions that yield sustained behavioral change. In R.J. DiClemente, R.A. Crosby, \& M.C. Kegler (Eds.). Emerging theories in health promotion practice and research: Strategies for improving public health (pp. 71-99). San Francisco: Jossey-Bass.

Petty, R.E., \& Cacioppo, J.T. (1986). Communication and persuasion: Central and peripheral routes to attitude change. New York: Springer-Verlag.

Petty, R.E., \& Cacioppo, J.T. (1986a). The elaboration likelihood model of persuasion. Advances in Experimental Social Psychology. 19, 124-205.

Plesko, M., Cotugna, N.. \& Aljadir, L. (2000). Usefulness of a brief fruit and vegetable FFQ in a college population. American Journal of Health Behavior, 24, 201-208.

Preston. M.A., Baranowski. T., \& Higginbotham. J.C. (1988). Orchestrating the points of community intervention: Enhancing the diffusion process. International guarterly of Community Health Education, 9. 11-34.

Prochaska, J.O., DiClemente, C.C., \& Norcross, J.C. (1992). In search of how people change: Applications to addictive behaviors. American Psychologist, 47, 1102-1114.

Prochaska, J.O., \& Velicer. W.F. (1997). The transtheoretical model of health behavior change. American Journal of Health Promotion, 12, 38-48.

Prochaska, J.O., Velicer, W.F., Rossi, J.S., Goldstein, M.G., Marcus, B.H., Rakowski. W., Fiore, C., Harlow, L.L., Redding, C.A., Rosenbloom, D., \& Rossi, S.R. (1994). Stages of change and decisional balance for 12 problem behaviors. Health Psychology. 13. 39-46.

Raad voor de Volksgezondheid en Zorg. (2000). Patiênt en Internet IPatient and the Internet). Zoetermeer: Raad voor de Volksgezondheid en Zorg.

Rakowski, W.. Ehrich. B., Goldstein, M.G., Rimer, B.K., Pearlman. D.N., Clark. M.A., Velicer, W.F., \& Woolverton, H. (1998). Increasing mammography among woman aged 40-74 by use of a stage-matched tallored intervention. Preventive Medicine, 27. 748-756.

Reubsaet, A., Brug, J.. Kitslaar, J., Van Hooff, J.P., \& Van den Borne, H. (in press). The impact and evaluation of two school-based interventions on intention to register an organ donation preference. Health Education Research.

Riedstra, M., Hardeman, W., Pruyn, J.F.A., Aarnink, E.J.M., Van der Feen de Lille, J.C.J.F., \& Jonkers. R. (1992). De effecten van de tweede landelijke Let op Vet campagne, gehouden in 1992: Consumentenonderzoek. [The effects of the second national Fat Watch campaign in 1992; Consumer resarch|. (Report number: V 92.453). Zeist: TNO.

Ritterband. L.M.. Gonder-Frederick, L.A.. Cox. D.J., Clifton. A.D., West, R.W., \& Borow- 
itz, S.M. (2003). Internet interventions: In review, in use, and into the future. Profes: sional Psychology: Research and Practice, 34, 527-534.

Robinson, T.N., Patrick, K., Eng. T.R., \& Gustafson. D. (1998). An evidence-based approach to interactive health communication: A challenge to medicine in the information age. JAMA, 280, 1264-1269.

Rolls, B.J., \& Shide, D.J. (1992). The influence of dietary fat on food intake and body weight. Nutrition Reviews, 50, 283-290.

Ronda, G. (2003). The Dutch heart health community intervention 'Hartslag Limburg': An evaluation. Maastricht: Universiteit Maastricht.

Ronda, G., Van Assema, P., \& Brug, J. (2001). Stages of change. psychological factors and awareness of physical activity levels in the Netherlands. Health Promotion Inter: national, 16, 305-314.

Rossi, P.H., Freeman. H.E., \& Lipsey, M.W. (1999). Evaluation: A systematic approach. Thousand Oaks: Sage Publications.

Ryan, P., \& Lauver, D.R. (2002). The efficacy of tailored interventions. Journal of Nursing Scholarship. 34, 331-337.

Schram, D., Maas, I.A.M., Poos, M.J.J.C., \& Jansen. J. (2001). De bijdrage van leefstijlfactoren aan de sterfte in Nederland [The contribution of life-style factors on mortality in the Netherlands]. Tijdschrift voor Gezondheidswetenschappen, 79, 211-225.

Sheeran, P. (2002). Intention-behaviour relations: A conceptual and empirical review. In M. Hewstone. \& W. Stroebe (Eds.). European review of social psychology (Vol. 12, pp. 1-36). Chicester: Wiley

Sheeran, P., \& Abraham, C. (1995). The health belief model. In M. Conner, \& P. Norman (Eds.), Predicting health behaviour: Research and practice with social cognition models (pp. 23-61). Buckingham: Open University Press.

Skinner, C.S., Campbell, M.K., Rimer, B.K., Curry, S., \& Prochaska, J.O. (1999). How effective is tailored print communication? Annals of Behavioral Medicine, 21, 290298.

Skinner, C.S., Strecher, V.J., \& Hospers, H. (1994). Physicians' recommendations for mammography: Do tailored messages make a difference? American Journal of Public Health, 84, 43-49.

Smidts, A. (2002). Kijken in het brein: Over de mogelykheden van neuromarketing (Looking in the brain: On the possibilities of neuromarketingJ. Rotterdam: Erasmus Research Institute of Management.

Stafleu. A.. De Graaf, C., Van Staveren, W.A.. \& Schroots. J.J. (1991). A review of selected studies assessing social-psychological determinants of fat and cholesterol intake. Food guality and Preference, 3, 183-200.

Stout. P.A., Villegas, J., \& Kim. H. (2001). Enhancing learning through use of interactive tools on health-related websites. Health Education Research. 16, 721-733.

Strecher, V.J. (1999). Computer-tailored smoking cessation materials: A review and discussion. Patient Education and Counseling, 36, 107-117.

Strecher, V.J.. Greenwood, T., Wang. C., \& Dumont, D. (1999). Interactive multimedia and risk communication. Journal of the National Cancer Institute Monographs, 25. 134-139.

Strecher, V.J., Kreuter, M.. Den Boer. D.J., Kobrin, S., Hospers, H.J., \& Skinner, C.S. (1994). The effects of computer-tailored smoking cessation messages in family practice settings. The journal of Family Practice, 39, 262-268.

Street, R.L., Gold, W.R. \& Manning. T. (1997). Health promotion and interactive technol- 
ogy: Theoretical applications and future directions. Mahwah: Lawrence Erlbaum.

Suls, J.. \& Wheeler, L. (Eds.). (2000). Handbook of social comparison: Theory and research. New York: Kluwer Academic/Plenum Publishers.

Tigges, B.B. (2001). Affiliative preferences, self-change, and adolescent condom use. Journal of Nursing Scholarship, 33. 231-237.

Van Assema, P., Brug, H., Dolders, M., Kok, G., \& Steenhuis, 1. (1996). Misconceptie van vetconsumptie: Een kwalitatief onderzoek naar oorzaken [Misconception of fat consumption: A qualitative study of causes]. Tyjdschrift voor Gezondheidswetenschappen, 74, 347-355.

Van Assema, P., Brug. J., Kok, G., \& Brants, H. (1992). The reliability and validity of a Dutch questionnaire on fat consumption as a means to rank subjects according to individual fat intake. European Journal of Cancer Prevention, 1. 375-380.

Van Assema, P., Brug. J., Ronda, G., \& Steenhuis, I. (2001). The relative validity of a short Dutch questionnaire as a means to categorize adults and adolescents to total and saturated fat intake. Journal of Human Nutrition and Dietetics, 14, 377-390.

Van Assema, P., Brug. J., Ronda, G., Steenhuis, I., \& Oenema, A. (2002). A short Dutch questionnaire to measure fruit and vegetable intake: Relative validity among adults and adolescents. Nutrition and Health, 16, 85-106.

Van den Brandt, P.A., Dagnelie, P.C.. \& Von Meyenfeldt. M.F. (1999). Voeding en kanker: causale, beschermende en therapeutische aspecten [Nutrition and cancer: causal. preventive and therapeutic aspects]. Nederlands Tijdschrift voor Geneeskunde, 143. 1414-1420.

Van Dijck, J.A.A.M., Coebergh, J.W.W., Slesling, S., \& Visser, O. (Eds.) (2002). Trends of cancer in the Netherlands 1989-1998. Utrecht: Vereniging van Integrale Kankercentra.

Van Duyn, M., Kristal, A., Dodd, K., Campbell, M.K., Glanz, K., \& Subar, A. (2001). Association of diet-related psychosocial variables with fruit and vegetable consumption: A national survey. American Journal of Health Promotion, 16, 69-78.

Van Oers, J.A.M. (Ed.) (2002). Gezondheid' op koers? Volksgezondheid toekomst verkenning 2002 /Health on course? Key messages from the 2002 Dutch public health status and forecast report). (RIVM-report number: 270551001). Houten: Bohn Stafleu van Loghum.

Van Wechem, S.N., Brug. J., Van Assema, P., Kistemaker, C., Riedstra, M., \& Lōwik. M.R.H. (1998). Fat watch: A nationwide campaign in the Netherlands to reduce fat intake - effect evaluation. Nutrition and Health, 12, 119-130.

Vereniging van Integrale Kankercentra (VIKC)، (2003), Kerngegevens over kanker in Nederland [Cancer in the Netherlands in fugures: Key figures]'. Available from: URL: http: //www.ikc.nl/vvik/index.html. Accessed: December 15, 2003.

Visser, O., Coebergh, J.W.W., Van Dijck, J.A.A.M., \& Siesling, S. (Eds.) (2002). Incidence of cancer in the Netherlands 1998. Utrecht: Vereniging van Integrale Kankercentra.

Voedingscentrum. (1998). Zo eet Nederland 1998 [How the Dutch eat 1998]. Den Haag: Voedingscentrum.

Voedingscentrum. (1999). Projectverslag eettesten [Project report self-tests]. Den Haag: Voedingscentrum.

Weinstein, N.D. (1980). Unrealistic optimism about future life events. Journal of Personality and Social Psychology. 39, 806-820.

Weinstein, N.D. (1988). The precaution adoption process. Health Psychology. 7. 355386. 
Weinstein, N.D., \& Klein, W.M. (1995), Resistance of personal risk perceptions to debiasing interventions. Health Psychology, 14, 132-140.

Weinstein. N.D., Rothman. A.J., \& Sutton. S.R. (1998). Stage theories of health behavior: Conceptual and methodological issues. Health Psychology. 17, 290-299.

Weinstein. N.D.. \& Sandman. P.M. (1992). A model of the precaution adoption process: Evidence from home radon testing. Health Psychology, 11, 170-180.

Weinstein, N.D., \& Sandman, P.M. (2002). Reducing the risks of exposure to radon gas: An application of the precaution adoption process model. In D. Rutter, \& L. Quine (Eds.). Changing health behaviour: Intervention and research with social cognition models (pp. 66-86). Buckingham: Open University Press.

Wheeler, L.. \& Miyake, K. (1992). Social comparison in everyday life. Journal of Personality and Social Psychology. 62, 760-773.

Willet. W.C. (1994). Diet and health: What should we eat? Science, 264, 532-537.

Windsor, R., Baranowski. T., Clark. N.. \& Cutter, G. (1994). Evaluation of health promotion health education and disease prevention programs. Mountain View: Mayfield.

Winzelberg. A.J., Eppstein. D., Eldredge, K.L., Wilfley. D.. Dasmahapatra, R., Dev. P., \& Taylor, C.B. (2000). Effectiveness of an Internet-based program for reducing risk factors for eating disorders. Journal of Consulting and Clinical Psychology. 68, 346350 .

Wood, J.V. (1989). Theory and research concerning social comparisons of personal attributes. Psychological Bulletin, 106, 231-248.

Wood. J.V. (1996). What is social comparison and how should we study it? Personality and Social Psychology Bulletin, 22, 520-537.

Wood, J.V.. Taylor, S.E., \& Lichtmann, R.R. (1985). Social comparison in adjustment to breast cancer, Journal of Personality and Social Psychology, 49, 1169-1183.

World Cancer Research Fund. (1997). Food, nutrition and prevention of cancer: A global perspective. Washington: World Cancer Research Fund and American Institute for Cancer Research.

World Health Organization. (2003). Diet, nutrition and the prevention of chronic diseases. (WHO Technical report Series 916), Geneva: World Health Organization.

Zock. P.L. (2001), Dietary fats and cancer. Current Opinion in Lipidology, 12, 5-10. 


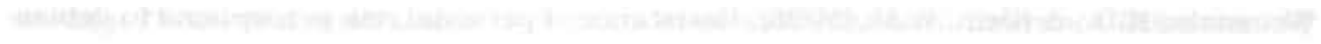

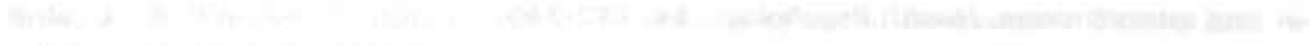

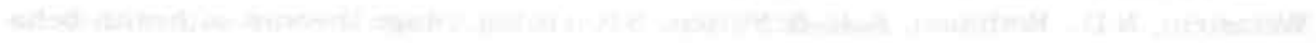

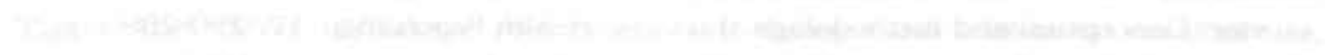

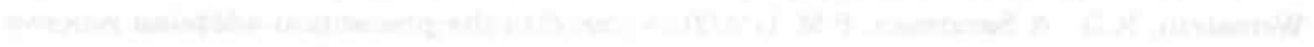
- 


\section{Summary}

This thesis presents studies on the efficacy of various intervention strategies aimed at decreasing saturated fat intake and increasing fruit and vegetable intakes. The interventions specifically focus on increasing awareness of personal intake levels of saturated fat, fruit and vegetables.

In Chapter 1, following the steps of planned health education, cardiovascular diseases and cancer were identified as highly prevalent diseases and major causes of mortality in the Netherlands. High saturated fat intake and low fruit and vegetable intakes are associated with an increased risk for these diseases and diets high in saturated fat and low in fruit and vegetables are very common in the Netherlands. An analysis of behavioural determinants revealed that lack of awareness of personal intake of saturated fat, fruit and vegetables is a barrier for the intention to change these intakes. Therefore, it is important to develop interventions that aim to increase awareness. Feedback and social comparison information were identified as potential methods for increasing awareness. Self-test forms and web-based computer tailored nutrition information were chosen as interventions to provide feedback. Positive and negative role model stories were chosen as a practical strategy to provide social comparison information.

Chapter 2 provides an overview of the history, the rationale and the technique of computer tailoring and the effects of computer-tailored nutrition education. Most studies of computer-tailored nutrition education tested tailored print materials. In general, such print-delivered computer-tailored nutrition information was found to be more effective in inducing dietary change than generic nutrition education, especially for reducing fat intake. More advanced applications of computer tailoring such as interactive, computer-based and Internet-based interventions may provide new opportunities and new challenges to computer-tailored nutrition education. Based on this overview it is concluded that more research is needed on how. when. why. where, for whom and through which channel of delivery. computer-tailored nutrition information is effective. 
Chapter 3 reports on a study in which the web-based computer tailored nutrition intervention is evaluated for its immediate effects on self-rated intake and intention to change fat, fruit and vegetable intakes. In a two-group, pre-test-post-test randomised controlled trial the taflored intervention was compared with generic nutrition information. The tailored intervention had significant effects on self-rated intakes and on the intention to eat less fat and more fruit and vegetables. The tailored intervention was rated as more personally relevant, more individualised and newer than the generic information. The web-based. tailored intervention was rated equally attractive by computer-literate and computer-illiterate respondents.

Chapter 4 describes a study in which the web-based tailored intervention and the self-test form intervention were evaluated for their immediate effects. In a randomised controlled trial the taflored intervention and the self-test forms were compared to generic nutrition information. Differential effects of the interventions for higher and medium or lower educated respondents were studied. Respondents who received tallored information were more likely to be aware of their fruit intake. Medium and lower educated respondents in the tailored intervention group were more aware of their fat intake levels. Respondents in the tailored intervention group had a significantly greater intention to change fat and fruit intake. No significant effects for the self-test form intervention were found. The tailored intervention was more likely to be used again and was rated as more personally relevant, more individualised and more new than both other interventions.

Short-term efficacy of the web-based computer-tailored intervention was evaluated in a three-group randomised controlled trial (Chapter 5). The web-based tailored intervention was compared with web-based generic information and no information. Three weeks post-intervention, respondents in the tailored intervention group were more aware of their fat and vegetable intake, and had a greater intention to change fat and vegetable intakes compared to those in the other two groups. These findings applied to the total studied population, as well as to the 'at risk' and 'unaware' subgroups. In the 'at risk' and 'unaware' subgroups, respondents in the tailored intervention group had a greater intention to change fruit intake and a higher fruit intake. Participants evaluated the tailored information more favourably than the generic information. Perceived personal relevance, individualisation and interestingness of the information mediated part of the effects of the tailored intervention.

Chapter 6 describes the study of the longer-term effects of the tailored intervention. The tailored intervention was compared to web-based generic nutrition information and to no information six months post-intervention. In this trial the focus was particularly on respondents with baseline intake levels not in agreement with recommendations (at risk group). Respondents in the tailored intervention group had a higher level of awareness of their vegetable intake and had a higher score for intention to change fat and vegetable intake than those in the other groups. Respondents in the tailored intervention group had a higher vegetable intake than respondents in the no information group.

Two studies explored social comparison information and modelling as meth- 
ods for increasing awareness and intention to change and for inducing behaviour change. A first exploratory cross-sectional study (Chapter 7), was conducted to gain more insight into social comparisons related to dietary fat intake. Many respondents could give examples of situations in which they compared themselves with others, whom they compare themselves with, and what aspects they observe in others. Comparisons were mainly made with close others such as partners, relatives and friends. Respondents who intended to change and younger respondents were more likely to compare themselves with others in relation to fat intake.

Chapter 8 describes a study into the effects of positive and negative role model stories on awareness, intention and fruit intake, in a group of respondents with low fruit intakes. The study was conducted as a randomised controlled trial, comparing the positive and the negative role model story with a control group. No effects were found for the positive or negative role model stories on awareness, intention to change or fruit intake. Stronger identification with the model and greater perceived credibility of the information was found for the negative role model story.

In the general discussion (Chapter 9) it is concluded that the web-based computer-tailored nutrition intervention was effective in increasing awareness and intention to change. Actual behaviour change was only marginally induced by the tailored intervention. More research is needed before interventions based on social comparison information can be used effectively in nutrition education. 



\section{Samenvatting}

In dit proefschrift worden studies gepresenteerd waarin verschillende interventiestrategieèn om de consumptie van verzadigd vet te verlagen en die van groente en fruit te verhogen, worden ontwikkeld en geèvalueerd. De interventies zijn er specifiek op gericht om mensen bewuster te maken van hun eigen consumptie van verzadigd vet, groente en fruit, als eerste essentiêle stap naar gedragsverandering.

In hoofdstuk 1 worden aan de hand van de stappen van het proces van planmatige gezondheidsvoorlichting hart- en vaatziektes en kanker geïdentificeerd als belangrijke oorzaken van ziektelast en sterfte in Nederland. Een hoge consumptie van verzadigd vet en een lage consumptie van groente en fruit hangen samen met een groter risico op het ontstaan van deze ziektes. In Nederland wordt meer verzadigd vet en minder groente en fruit gegeten dan wordt aanbevolen. Uit een analyse van gedragsdeterminanten bleek dat gebrek aan bewustzijn van de eigen consumptie van verzadigd vet, groente en fruit een belangrijke barrière is voor de motivatie om minder verzadigd vet en meer groente en fruit te gaan eten. Daarom is het belangrijk om interventies te ontwikkelen die er in eerste instantie op zijn gericht om mensen beter bewust te maken van wat ze eten. Feedback en sociale vergelijkingsinformatie zijn methodes die kunnen worden gebruikt om mensen beter bewust te maken van wat ze eten. Zelftesten en web-based advies-op-maat zijn gekozen als interventies om feedback te geven en positieve en negatieve rolmodelverhalen om sociale vergelijkingsinformatie te geven. Deze interventies werden in verschillende studies geêvalueerd.

Hoofdstuk 2 geeft een literatuuroverzicht van de techniek en de effecten van voedingsadvies-op-maat. In de meeste eerdere onderzoeken naar die effecten werden schriftelijke versies van voedingadvies-op-maat getest. Schriftelijk voedingsadviesop-maat is in het algemeen effectiever in het veranderen van voedingsgedrag. met name in het verminderen van de vetconsumptie, dan algemene voedingsinformatie. Meer geavanceerde en interactieve toepassingen van advies-op-maat, waarbij een advies-op-maat programma kan worden gebruikt op de computer of via internet. 
bieden nieuwe mogelijkheden en nieuwe uitdagingen voor voedingsadvies-op-maat. In dit proefschrift wordt voor het eerst systematisch onderzoek beschreven naar de effecten van zo'n nieuwe toepassing, namelijk een web-based voedingsadvies-opmaat, dat kan worden verspreid via het internet.

Hoofdstuk 3 laat de resultaten zien van een gerandomiseerde gecontroleerde studie naar de directe effecten van het web-based voedingsadvies-op-maat op de eigen inschatting van de vet-, groente- en fruitconsumptie en op de intentie om minder vet, meer groente en meer fruit te gaan eten. Hierbij werd het voedingsadvies-op-maat vergeleken met algemene voedingsinformatie. Het advies-op-maat had een significant effect op de inschatting van de eigen vet-, groente- en fruitconsumptie en op de intentie om minder vet en meer groente en fruit te gaan eten. Het advies-op-maat werd als meer persoonlijk relevant, meer geindividualiseerd en nieuwer beoordeeld dan de algemene informatie. Zowel mensen met als zonder computervaardigheden vonden het web-based advies op maat aantrekkelijk om te gebruiken.

In Hoofdstuk 4 wordt gekeken naar de directe effecten van het advies-op-maat en de zelftesten. In een gerandomiseerd gecontroleerd experiment worden het advies op maat en de zelftesten vergeleken met schriftelijke algemene voedingsinformatie. Mensen die advies op maat kregen, waren meer bewust van hun fruitconsumptie dan de mensen die algemene voedingsinformatie kregen. Zij waren ook significant vaker van plan om minder vet en meer fruit te gaan eten. Verder waren middel en laag opgeleide mensen die advies-op-maat kregen meer bewust van hun vetconsumptie. De zelftesten bleken niet effectiever dan de algemene voedingsinformatie. Het advies-op-maat werd als meer persoonlijk relevant, geĭndividualiseerd en nleuw beoordeeld dan de zelftesten en de algemene voedingsinformatie. Mensen die advies op maat kregen gaven vaker aan dat zij het advies opnieuw zouden gebruiken dan mensen die de zelftesten of algemene informatie kregen.

De kortetermijneffecten van het web-based advies, op maat werden geēvalueerd in een gerandomiseerd gecontroleerd experiment met drie groepen (hoofdstuk 5). Het web-based advies op maat werd vergeleken met web-based algemene voedingsinformatie en mel geen informatie. Mensen die advies-op-maat kregen waren drie weken na die interventie meer bewust van hun vet- en groenteconsumptie en meer van plan om minder vet en meer groente te gaan eten, dan mensen die geen of algemene voedingsinformatie kregen. Deze resultaten werden gevonden in de totale onderzoeksgroep, maar ook in de subgroep van mensen die op de voormeting meer vet of minder groente en fruit aten dan wordt aanbevolen en in de subgroep van mensen die hun vetconsumptie onderschatten of hun groente en fruit consumptie overschatten. In deze subgroepen waren mensen die advies op maat kregen ook meer van plan om meer fruit te gaan eten en was de fruitconsumptie hoger. Respondenten waardeerden het advies op maat beter dan de algemene voedingsinformatie. Of de informatie als persoonlijk relevant, geïndividualiseerd of interessant werd ervaren, bleek een rol te spelen bij het ontstaan van de effecten van het advies op maat.

In hoofdstuk 6 wordt een studie beschreven naar de langeretermijneffecten van 
het advies op maat. In dit experiment werd specifiek gekeken naar mensen van wie de consumptie van vet, groente en fruit op de voormeting niet in overeenstemming was met de aanbeveling. Het advies op maat werd vergeleken met web-based algemene voedingsinformatie en met geen informatie. Zes maanden na de interventie waren mensen die advies-op-maat kregen meer bewust van hun groenteconsumptie en meer van plan om minder vet en meer groente te gaan eten, dan mensen die algemene voedingsinformatie of geen informatie kregen. Mensen die advies-opmaat kregen aten iets meer groente dan mensen die geen informatie kregen.

De hoofdstukken 7 en 8 besteden aandacht aan sociale vergelijkingsinformatie en modelling als methodes om verandering in bewustzijn, in intentie om te veranderen en in gedrag te bewerkstelligen. In een explorerende studie (hoofdstuk 7) is onderzocht of en hoe mensen hun eigen vetconsumptie vergelijken met die van anderen. Veel respondenten konden voorbeelden geven van situaties waarin ze zich vergelijken met anderen. met wie ze zich vergelijken en op welke aspecten ze letten om zich te vergelijken. Mensen vergeleken zich hoofdzakelijk met naasten, zoals partners, familieleden en vrienden. Respondenten die van plan waren om minder vet te gaan eten en jongere respondenten vergeleken zich meer met anderen dan respondenten die niet van plan waren te veranderen en oudere respondenten.

Hoofdstuk 8 beschrijft de resultaten van een studie naar de effecten van positieve en negatieve rolmodelverhalen op bewustzijn. intentie om te veranderen en fruitconsumptie, bij mensen die weinig fruit eten. In dit gerandomiseerde, gecontroleerde experiment werden de effecten van blootstelling aan positieve of negatieve rolmodelverhalen over fruitconsumptie vergeleken met blootstelling aan een rolmodelverhaal dat niet over fruitconsumptie ging. De positieve of negatieve rolmodelverhalen hadden geen effect op bewustzijn, de intentie om meer fruit te gaan eten of op de consumptie. Wel bleken respondenten zich sterker met het negatieve rolmodel te identificeren en het negatieve rolmodel geloofwaardiger te vinden.

Het proefschrît sluit af met een algemene discussie (hoofdstuk 9), De conclusie is dat feedback met behulp van het web-based voedingsadvies-op-maat geschikt is als interventie om bewustzijn van de vet-, groente- en fruitconsumptie te verhogen en mensen te motiveren om minder vet en meer groente en fruit te gaan eten. Er is meer onderzoek nodig voordat interventies die gebruik maken van sociale vergelijkingsinformatie effectief kunnen worden toegepast in voedingsvoorlichting. 


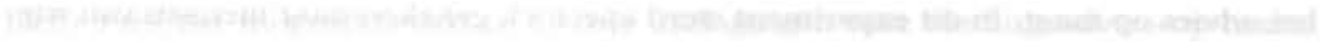

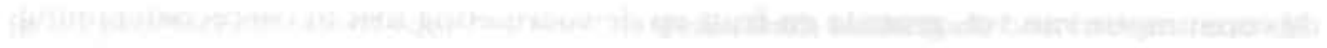

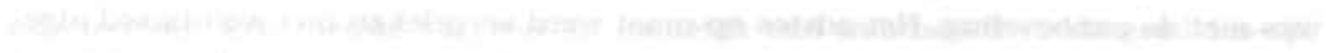

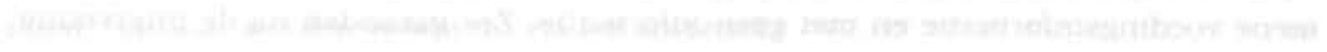

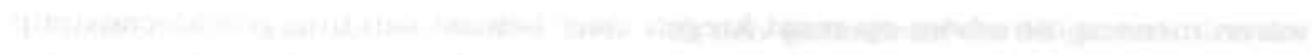

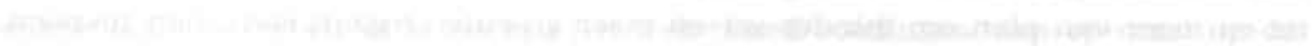

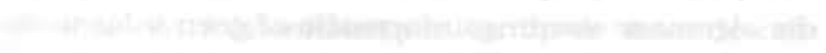

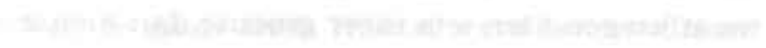

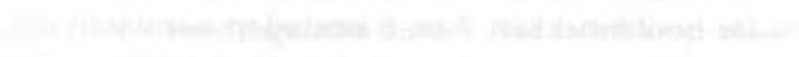

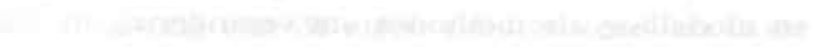

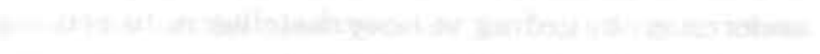

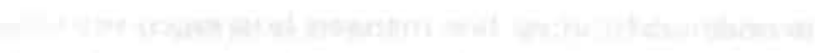

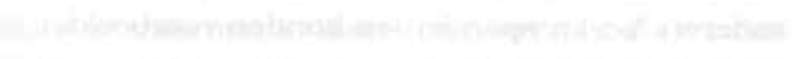

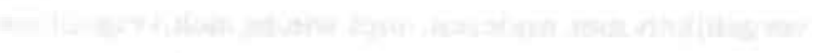
-

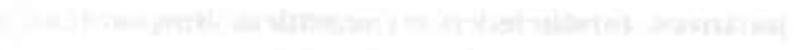

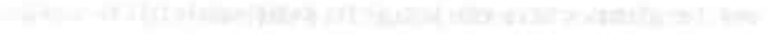

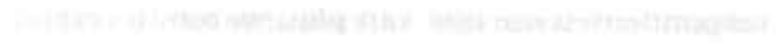
and

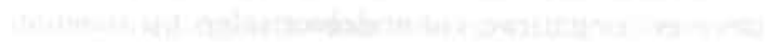

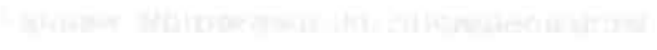

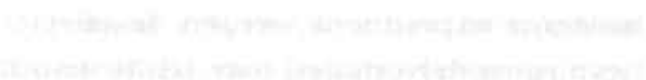




\title{
Dankwoord
}

Het laatste woord dat ik schrijf in dit proefschrift is

\author{
Bedankt!!
}

Bedankt Hans, voor je inspirerende begeleiding. Ik had me geen betere begeleider kunnen wensen. Je geloofde in mij. stond altijd open voor mijn ideeên, gaf me de ruimte en kon omgaan met mijn twijfels. Ik heb veel van je geleerd. Het was en is nog steeds erg prettig om met je samen te werken.

Bedankt collega's uit Maastricht, voor de prettige tijd die ik mede dankzij jullie in Maastricht heb gehad. Het was een erg fijne omgeving voor mijn eerste schreden op het onderzoekspad. Ik denk met veel plezier terug aan de tijd in Maastricht en mis de gesprekken en discussies over theorie, model en onderzoeksveld.

Bedankt iedereen die heeft bijgedragen aan het onderzoek. De deelnemers. Degenen die zich bij de bedrijven hebben ingezet om deelnemers te kunnen werven. Angélique van de Kar en de andere medewerkers van Hartslag Midden Limburg voor de samenwerking in de veldstudie. Harold Dello en Mark Lardinois voor het ontwikkelen van de programmatuur om web-based advies op maat te genereren. Alle assistenten die hebben geholpen bij de uitvoering van de praktische zaken. De medewerkers van Cendris in Heerlen voor het invoeren van de gegevens. De leden van de adviescommissie, voor de inbreng en de belangstelling.

Bedankt allen die hebben bijgedragen aan het schrijven, bundelen en becommentariëren van de artikelen en teksten. Jan Klerkx en Katrina Giskes voor het corrigeren van het Engels.

Bedankt collega's en kamergenoten in Rotterdam, voor de steun en het medeleven bij een nieuwe start en de laatste loden van het afronden van het proefschrift naast een nieuwe baan.

Bedankt Johan en Trijntje dat jullie mij terzijde willen staan bij het voorbereiden voor en het verdedigen van het proefschrift.

Last but not least, lieve vrienden en familie. Bedankt voor alle steun door grote of kleine dingen, maar vooral 'for just being there'.

Anke 
bichomatroc 


\section{Curriculum vitae}

Anke Oenema was born on July 20, 1966 in Oosterend, Hennaarderadeel, In 1983 she received her HAVO diploma at the Jan Brugman College in Bolsward. In that same year she started her higher professional training in nursing at the Academie voor Gezondheidszorg Noord Nederland in Groningen, where she graduated in 1988 with first class honours. From 1989 till 1998 she worked as a registered nurse at the Martini Ziekenhuis in Groningen. In 1995 she started her study in Health Education and Promotion at the Faculty of Health Sciences of the Universiteit Maastricht, and graduated in 1998 with first class honours. In 1999 she started her work on the research project resulting in the studies described in this thesis, as a PhD fellow at the department of Health Education and Promotion of the Universiteit Maastricht. In January 2003 she began working as a post-doc researcher at the Department of Public Health of the Erasmus University Medical Centre in Rotterdam, continuing her work on the development and evaluation of (computer-tailored) health promotion interventions. 
(a) 